EXTENDED REPORT

\title{
Unexplained head tilt following surgical treatment of congenital esotropia: a postural manifestation of dissociated vertical divergence
}

\author{
M C Brodsky, R Jenkins, P Nucci
}

Br J Ophthalmol 2004;88:268-272. doi: 10.1136.bjo.2003.021055

See end of article for authors' affiliations

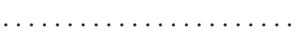

Correspondence to: Dr M C Brodsky, Arkansas Children's Hospital, 800 Marshall, Little Rock, Arkansas 72202, USA; brodskymichaelc@ vams.edu

Accepted for publication 17 June 2003

\begin{abstract}
Background: Strabismus surgery for congenital esotropia can be complicated by the development of a postoperative head tilt.

Purpose: To determine the pathophysiology of acquired head tilting following horizontal realignment of the eyes in children with congenital esotropia.

Materials and methods: Retrospective analysis of nine children with congenital esotropia who developed unexplained head tilts following horizontal realignment of the eyes.

Results: Shortly after strabismus surgery, each child developed a head tilt in association with asymmetrical dissociated vertical divergence (DVD). Five children maintained a head tilt toward the side of the fixing eye (group 1), which did not serve to control the DVD. Four children maintained a head tilt toward the side of the hyperdeviating eye, which served to control the DVD (group 2). Children in group 2 had earlier horizontal muscle surgery and developed better stereopsis than those in group 1, suggesting that the higher degree of single binocular vision and stereopsis in these children may have led to a compensatory torticollis to control an asymmetrical DVD.

Conclusions: The onset of an unexpected head tilt after congenital esotropia surgery is usually a postural manifestation of asymmetrical DVD. In this setting, a head tilt toward the side of the fixing eye corresponds with a postural manifestation of the underlying central vestibular imbalance that produces DVD, while a head tilt toward the side of the hyperdeviating eye serves to counteract the hyperdeviation and stabilise binocular vision.
\end{abstract}

C ongenital esotropia is characterised by a large angle crossing of the eyes that begins within the first six months of life. ${ }^{1}$ Associated ocular motility disturbances such as inferior oblique overaction, latent nystagmus, and dissociated vertical divergence (DVD) may first appear following surgical realignment of the eyes. ${ }^{12}$ Less commonly, a head tilt develops after successful horizontal realignment of the eyes. ${ }^{3}$ This unexpected finding is often perplexing to the surgeon and frustrating to the parents, who complain that "the eyes are straight, but now he tilts his head all the time". In an attempt to elucidate the pathophysiology of this complication, we describe nine children with congenital esotropia who developed unexplained head tilts following successful horizontal realignment of the eyes.

\section{MATERIALS AND METHODS}

Medical records from children examined at Arkansas Children's Hospital and Texas Children's Hospital Eye Clinic, and San Paolo Hospital were retrospectively reviewed for unexplained head tilting following congenital esotropia surgery. After exclusion of patients with congenital nystagmus, cyclovertical muscle palsy, or primary oblique muscle overaction with baseline fundus torsion, nine children were found who met inclusion criteria. All nine children had been examined on multiple occasions. Historical findings included age at the time of strabismus surgery, surgical procedure performed, age at which the head tilt was first noted, age at which the DVD was first noted, presence or absence of a superimposed hypertropia, nature of strabismus surgery used to treat the head tilt, and surgical or non-surgical outcome.

A standard technique was used to measure the DVD and any associated hyperdeviation. The amplitude of DVD in each eye was measured by occluding the eye and adding base down prism behind the occluder until no hyperdeviation was observed when the occluder was shifted to the other eye. A superimposed hyperdeviation was detected by measuring a hypotropia of the other eye using alternate cover testing.

Sensory testing, occlusion testing, placement of prisms to match the vertical deviation, and Bielschowsky head tilt testing were used to determine whether the head tilt served to improve vertical alignment of the eyes and stabilise binocular vision. Sensory testing was performed with the head held in the compensatory position. A patient's head tilt was designated as compensatory for binocular vision when it improved vertical alignment of the eyes, and non-compensatory for binocular vision when it persisted despite of vertical misalignment of the eyes, when a contralateral head tilt restored improved vertical alignment, or when amblyopia was present in one eye.

\section{RESULTS}

Nine children developed enigmatic head tilts following horizontal strabismus surgery for congenital esotropia. In five children (group 1), the head tilt was directed towards the side of the fixing eye. In four children (group 2) the head tilt was directed towards the side of the hyperdeviating eye. In one child (case 3), a head tilt had been documented preoperatively and it became more conspicuous to parents and physicians after bimedial rectus muscle recessions were performed to treat congenital esotropia. In the other children, the onset of the postoperative head tilt usually preceded the detection of an asymmetrical DVD with a spontaneous hyperdeviation

Abbreviations: DVD, dissociated vertical divergence 
of one eye. In all children, the amplitude of the DVD was smaller in the fixing eye than in the spontaneously hyperdeviating eye. The associated head tilts were larger in group 2 (range 10-30 degrees) than group 1 (range 5-15 degrees) and were present during both near and distance fixation.

Four of five patients in group 1, and two of four patients in group 2 had latent nystagmus. Only one patient in each group had a coexistent head turn toward the side of the fixing eye. No child had an associated A or V pattern, although an apparent inferior oblique overaction of one eye, as manifested by a spontaneous elevation when the eye was adducted, was often an early manifestation of DVD. In both groups, the head tilt was present to a similar degree during distance and near fixation. When the spontaneous head tilt was directed towards the side of the fixing eye (group 1), a manifest DVD persisted despite the head tilt. Three of the five children in group 1 (cases 1,3 , and 4) maintained a spontaneous head tilt toward the side of the fixing eye that was found to resolve when the fixating eye was patched but not when the hyperdeviating eye was patched. One child (case 4) maintained a head tilt toward the side of the fixing eye despite the fact that Bielschowsky head tilt testing toward the opposite side eliminated the hyperdeviation. When the spontaneous head tilt was directed toward the side of the hyperdeviating eye (cases 6-9), Bielschowsky head tilt testing indicated that the head tilt served to control the DVD.

In general, children with head tilts toward the side of the hyperdeviating eye had earlier horizontal strabismus surgery and better stereopsis than those with a head tilt toward the side of the fixating eye (table 1). Patient age at the time of initial strabismus surgery ranged from 11 months to 4 years for group 1 and 7 months to 1 year for group 2. No patient in group 1 developed more than gross stereopsis (Titmus fly) whereas two patients in group 2 developed 100 seconds of arc. Since the head tilt in group 1 was not compensatory for binocular vision, subsequent vertical strabismus surgery to treat the head tilt was performed only in group 2 . In one child in group 2 (case 6), the compensatory head tilt was reduced by vertical prisms and subsequently by vertical muscle surgery which reduced the asymmetry of the DVD in the two eyes. In one other patient in group 2 (case 8), vertical rectus muscle surgery reduced the size of the head tilt.

\section{DISCUSSION}

Previous studies have documented an association between the development of a head tilt in children with surgically corrected congenital esotropia and the presence of DVD. ${ }^{3-9}$ The curious finding in these cases is that the head tilt may be directed towards or away from the side of the fixating eye. This observation raises the question of whether the head tilt arises from an underlying central vestibular imbalance that alters the patient's vertical orientation, or whether it serves to inhibit the hyperdeviation in one eye and thereby stabilise binocular vision. Making this determination requires the acquisition of sensory data, field measurements, and head tilt testing, which can be difficult to obtain in young children. For this reason, it is often necessary to follow affected children longitudinally to establish the underlying cause of the head tilt.

Crone found head tilting in 26 of 113 children with DVD. ${ }^{4}$ In "the great majority", the head was tilted towards the shoulder on the side of the fixing eye. Crone proposed that this head tilt was compensatory for the incycloduction of the fixing eye. Since the hyperdeviation decreased rather than increased when the head was tilted toward the opposite shoulder, Crone concluded that "the quest for binocular vision is quite excluded as a cause of torticollis in all these cases." Lang found an abnormal head tilt in 29 of 82 children with congenital esotropia and DVD. ${ }^{3}$ In 18 children, the head was inclined toward the side of the fixing eye, while in 11 children the head was inclined toward the side of the hyperdeviating eye. Lang also noted that the head tilt was not adopted to avoid diplopia. ${ }^{3}$

In a more recent prospective study, Betchel et al found a manifest head tilt in 26/74 (35\%) of patients with DVD, no prior vertical muscle surgery, and an ocular fixation preference. ${ }^{5}$ Eighteen of $26(69 \%)$ of these patients had a head tilt toward the side of the hyperdeviating eye. In $19 \%$ of patients with manifest head tilts, the head tilt produced no qualitative or quantitative change in the DVD. In another prospective study of anomalous head postures with DVD by Santiago and Rosenbaum, twelve of 14 patients tilted their heads towards the side of the fixing eye, whereas two patients tilted their heads towards the side of the hyperdeviating eye. ${ }^{6}$ The authors noted that head tilting did not occur in their patients until after strabismus surgery. They postulated that surgical realignment permitted fusional mechanisms to function, and that head tilting was probably advantageous for fusion. In a series of consecutive cases with DVD, Prieto-Diaz et al found a head tilt in 26 patients $(49 \%) .^{78}$ The head tilt was directed towards the side of the fixing eye in 19 patients and towards the side of the hyperdeviating eye in seven patients. De Decker and Dannheim-de Decker noted a head tilt in 29 of 73 patients who were operated on for bilateral DVD. ${ }^{9}$ Nineteen patients had a head tilt toward the side of the fixing eye, and 10 had a head tilt toward the side of the hyperdeviating eye. These studies establish a dichotomy with respect to the direction of head tilt relative to the fixing eye, and the compensatory (that is, adapted to modulate the DVD and improve binocularity) versus non-compensatory (that is, centrally driven) nature of the tilt.

The evolutionary basis for DVD and its associated torticollis can be traced back to visuo-vestibular postural responses which are most pronounced in fish and insects. ${ }^{10-12}$ In the upright fish, unequal light input to the two eyes produces a body tilt in the roll (frontal) plane, causing the dorsal aspect to rotate toward the side with greater light input (dorsal light reflex). ${ }^{11}{ }^{12}$ In a vertically restrained fish, unequal visual input to the two eyes produces a vertical divergence of the eyes, with depression of the eye that has greater visual input and elevation of the eye that has less visual input. ${ }^{12}$ This primitive visuo-vestibular response orients the dorsal side of the animal toward the sky, and maintains equal visual input in the two eyes to maintain visual vertical orientation. ${ }^{13}$ DVD conforms to a human dorsal light reflex, which realigns the eyes to a tilted visual orientation when congenital esotropia precludes normal binocular visual development..$^{13-15}$

Invoking the human dorsal light reflex as the underlying cause for the head tilt in group 1 would explain why these patients maintain a head tilt despite a manifest hyperdeviation. ${ }^{3}$ In the same way that a primitive dorsal light reflex encourages a body tilt towards the side of the eye with greater visual input, a human dorsal light reflex would be expected to encourage a head tilt toward the side of the fixing eye. ${ }^{13}{ }^{14}$ Although we have designated this head tilt as noncompensatory for binocular vision, it is a compensatory postural adaptation at the central vestibular level in the sense that it serves to realign the head to a tilted internal representation of vertical (fig 1 , left)..$^{13}{ }^{14}$ This central vestibular imbalance may explain why one child in group 1 (case 4) maintained a left head tilt with a manifest hyperdeviation of the right eye despite the fact that the hyperdeviation resolved when the head was tilted to the right. A subjective visual tilt following occlusion of one eye has recently been shown in humans with DVD. ${ }^{15}$ 


\begin{tabular}{|c|c|c|c|c|c|c|}
\hline \multicolumn{7}{|c|}{ Group 1: Head tilt toward from side of fixing eye } \\
\hline Case & $\begin{array}{l}\text { Age BMR } \\
\text { performed }\end{array}$ & $\begin{array}{l}\text { Direction of head } \\
\text { tilt/age first noted }\end{array}$ & $\begin{array}{l}\text { DVD } \\
\text { characteristics }\end{array}$ & $\begin{array}{l}\text { Head tilt } \\
\text { stest }\end{array}$ & Sensory & $\begin{array}{l}\text { Response to patch } \\
\text { test and to treatment }\end{array}$ \\
\hline 1 & 4 years & Left/postop & $R>L$ & NP & $\begin{array}{l}\text { Bagolini - ARC; } \\
\text { Randot - no } \\
\text { stereopsis }\end{array}$ & $\begin{array}{l}\text { Head tilt resolved } \\
\text { with patching OS but } \\
\text { not OD }\end{array}$ \\
\hline 2 & 4 years & Left/postop & $R>L$ & NP & $\begin{array}{l}\text { Bagolini - ARC; } \\
\text { Randot - no } \\
\text { stereopsis }\end{array}$ & \\
\hline 3 & 21 months & Right/18 months & $L>R$ & NP & Titmus - negative & $\begin{array}{l}\text { Head tilt resolved } \\
\text { with patching OD but } \\
\text { not OS; symmetrical } \\
\text { SR rec - NI }\end{array}$ \\
\hline 4 & 12 months & Left/25 months & $R>L$ & $\begin{array}{l}\uparrow \text { RHT with } \\
\text { left head tilt; } \\
\text { RHT resolves } \\
\text { with right } \\
\text { head tilt }\end{array}$ & $\begin{array}{l}\text { Bagolini - ARC; } \\
\text { W4D - supp OD }\end{array}$ & $\begin{array}{l}\text { Head tilt resolved } \\
\text { with patching OS but } \\
\text { not OD }\end{array}$ \\
\hline 5 & 11 months & Right/18 months & $L>R$ & $\begin{array}{l}\text { LHT persists } \\
\text { with head tilt } \\
\text { to right }\end{array}$ & $\begin{array}{l}\text { Bagolini - ARC; } \\
\text { Titmus+fly W4D } \\
\text { fusion }\end{array}$ & $\begin{array}{l}\text { Head tilt resolved } \\
\text { with patching; LHT } \\
\text { resolved with LIOAP }\end{array}$ \\
\hline \multicolumn{7}{|c|}{ Group 2: Head tilt toward side of hyperdeviating eye } \\
\hline 6 & 11 months & Left/18 months & $L>R$ & $\begin{array}{l}\uparrow \text { LHT with } \\
\text { right head tilt } \\
\text { resolves with } \\
\text { left head tilt }\end{array}$ & $\begin{array}{l}\text { DMR - no torsion; } \\
\text { Bagolini - int supp } \\
\text { OD; Titmus } 100 \\
\text { sec/arc; W4D } \\
\text { vertical diplopia }\end{array}$ & $\begin{array}{l}\text { Vertical prisms } \\
\text { eliminated head tilt; } \\
\text { BSR+RIRres reduced } \\
\text { head tilt }\end{array}$ \\
\hline 7 & 7 months & Right/18 months & $R>L$ & $\begin{array}{l}\uparrow \text { RHT with } \\
\text { left head tilt; } \\
\downarrow \text { RHT with } \\
\text { right head tilt }\end{array}$ & $\begin{array}{l}\text { Bagolini - NRC; } \\
\text { W4D - alt supp; } \\
\text { Titmus+fly }\end{array}$ & NP \\
\hline 8 & 11 months & Right/postop & $R>L$ & $\begin{array}{l}\uparrow \text { RHT with } \\
\text { left head tilt; } \\
\downarrow \text { RHT with } \\
\text { right head tilt }\end{array}$ & $\begin{array}{l}\text { Bagolini - ARC } \\
\text { with int supp OS; } \\
\text { Titmus } 100 \mathrm{sec} / \\
\text { arc; W4D -int } \\
\text { supp OD }\end{array}$ & $\begin{array}{l}\text { Head filt decreased; } \\
\text { RLRrec; LIRrec }\end{array}$ \\
\hline 9 & 1 year & Right/postop & $R>L$ & $\begin{array}{l}\downarrow R H T \text { with } \\
\text { right head tilt; } \\
\uparrow R H T \text { with } \\
\text { left head tilt }\end{array}$ & Bagolini ARC & $\begin{array}{l}\text { Symmetrical BSR rec - } \\
\mathrm{NI}\end{array}$ \\
\hline \multicolumn{7}{|c|}{$\begin{array}{l}\text { M, male; F, female; - , unknown; NP, not performed; L, left; R, right; } \uparrow \text {, increased; } \downarrow \text {, decreased; DMR, Double } \\
\text { Maddox Rod; LHT, left hyperdeviation; RHT, right hyperdeviation; NI, no improvement; BMR, bimedial recession; } \\
\text { W4D, Worth Four Dot; sec/arc, seconds of arc; NRC, normal retinal correspondence; ARC, anomalous retinal } \\
\text { correspondence; int supp, intermittent suppression; alt supp, alternate suppression; IOAP, inferior oblique } \\
\text { anteroplacement; SV, subjective vertical; postop, noted shortly after surgery; rec, recession; res, resection. }\end{array}$} \\
\hline
\end{tabular}

Some have questioned whether a head tilt toward the side of the fixing eye could serve to improve visual acuity by damping latent nystagmus. ${ }^{16}{ }^{17}$ This mechanism seems implausible since any static ocular counterroll would primarily damp the torsional component of latent nystagmus, which does not displace the image of regard from the fovea and therefore does not degrade visual acuity. ${ }^{18}$ In this group, a head turn towards the side of the fixing eye would be the necessary strategy to lessen the horizontal component of latent nystagmus, stabilise foveation, and improve monocular visual acuity. Lang ${ }^{3}$ reported that patients corresponding to group l commonly have a head turn toward the side of the fixing eye. Although four children in our group 1 had latent nystagmus, only one child had a coexisting head turn towards the side of the fixing eye. It is unlikely that the other three patients with latent nystagmus were maintaining a head tilt to selectively reduce the torsional component in the absence of a head turn to reduce the horizontal component.

The DVD associated head tilts in group 2 present a different set of diagnostic challenges. In these patients, it may be difficult to distinguish a manifest hyperdeviation from an occlusion hyperphoria since the compensatory head tilt serves to neutralise the manifest hyperdeviation. Furthermore, Bielschowsky head tilt testing is particularly difficult to quantify in young children with bilateral DVD. Therefore, a manifest hyperdeviation must be carefully sought after the patient's head has been placed in the upright position. Only when a manifest hyperdeviation of one eye can be identified, the amplitude of the DVD in both eyes measured, and Bielschowsky head tilt testing performed, can the compensatory nature of the postoperative head tilt be established.

Jampolsky has characterised the Bielschowsky head tilt response in DVD as one in which a head tilt to either side increases the hyperdeviation of the contralateral eye. ${ }^{19} 20$ This response, which is opposite to that seen in patients with superior oblique palsy, ${ }^{19}$ reflects the additive effects of utricular stimulation and oblique muscle innervation associated with DVD. ${ }^{13}{ }^{14}$ When unequal visual input evokes a hyperdeviation of the left eye, for example, visuo-vestibular innervation activates the right superior and left inferior oblique muscles to produce a cyclovertical divergence ${ }^{13}$ (fig 1 , right). This cyclovertical divergence then necessitates secondary fixational innervation to maintain monocular fixation with the lower (visually preferred) eye..$^{17} \mathrm{~A}$ dorsal light reflex in humans would cause the head to tilt away from the side of the hyperdeviating eye (fig l, left) which would increase the left hyperdeviation as a right head tilt activates otolithic innervation predominantly to the right superior 


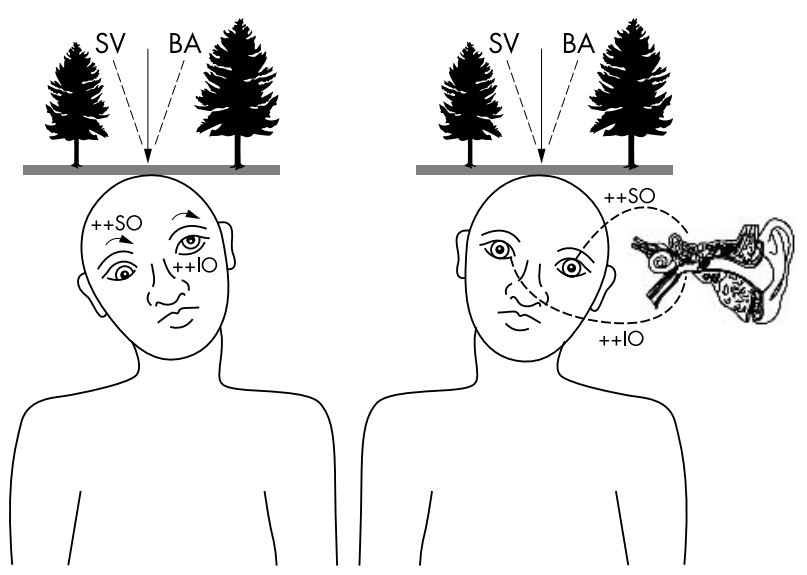

Figure 1 Components of the head tilt response in DVD. Left: head tilt toward the side of the fixing eye (group 1). A human dorsal light reflex induces a tilt of the subjective vertical (SV) towards the side of the fixing eye and a vertical divergence of the eyes. A head tilt to align the head with the tilted subjective vertical would be necessary to maintain vertical orientation. Right: compensatory head tilt in DVD. A patient with DVD and a hyperdeviation of the left eye (left figure) can use a compensatory head tilt to the left to recruit otolithic innervation to neutralise the DVD innervation and nullify the existing vertical divergence and restore binocular alignment (BA) of the eyes.

oblique and left inferior oblique muscles. ${ }^{13}{ }^{14}$ Conversely, a head tilt to the left would recruit otolithic innervation predominantly to the right inferior oblique and left superior oblique muscles, which serve to neutralise a left DVD associated hyperdeviation and improve vertical alignment (fig l, right). Thus, by tilting the head towards the side of the more hyperdeviating eye, children in group 2 could calibrate their head position to modulate DVD in the two eyes and thereby stabilise binocular fusion. ${ }^{13}{ }^{14}$ Both patients who underwent strabismus surgery in an attempt to equalise the hyperdeviations showed reduction in the size of the head tilt and displayed some residual asymmetry in the hyperdeviations between the two eyes.

According to Jampolsky, DVD can eventually lead to secondary superior rectus contracture in the hyperdeviating eye. ${ }^{20}$ Superior rectus contracture can reverse the head tilt response in DVD, causing a hyperdeviation in the affected eye to increase with ipsilateral head tilt and decrease with contralateral head tilt. ${ }^{20}$ In this setting, a compensatory head tilt to the side of the fixating eye may be used to minimise otolithic innervation to the tight superior rectus muscle, while a head tilt towards the side of the higher eye will augment the hyperdeviation. When a superior rectus contracture develops, surgical recession of the tight superior rectus muscle can reduce or eliminate the compensatory head tilt. ${ }^{6}$

Only one of our patients in group 1 developed evidence of a superior rectus contracture (case 5), as evidenced by a head tilt away from the hyperdeviating eye and an induced hypotropia of the fixating eye when it was patched. Although three of our patients in group 2 had a superimposed hypertropia, they manifested a head tilt towards the side of the hyperdeviating eye, which is inconsistent with superior rectus muscle contracture. It therefore appears that a superimposed hypertropia can develop in some patients with asymmetrical DVD without other clinical signs of superior rectus contracture. It is also possible that these patients had a small coexistent hypertropia that was unrelated to the DVD (perhaps caused by unequal vertical positioning of the medial rectus muscles during surgery or by undetected unilateral inferior oblique muscle overaction). In a young child, a bilateral symmetrical DVD could mask a small hypertropia to produce a clinical appearance that simulates asymmetrical DVD. Figure 2 summarises the probable mechanisms by which DVD can manifest with head tilting in children with surgically treated congenital esotropia.

The dichotomy in the direction of head tilt relative to the fixing eye in this study suggests asymmetrical DVD induces a schizophrenic situation in which the need for vertical orientation and the need for vertical ocular alignment create conflicting postural drives. One the one hand, a head tilt towards the side of the fixing eye that is necessary to reestablish vertical orientation will increase the hyperdeviation of the contralateral eye (fig l, left). On the other hand, a head tilt towards the side of the hyperdeviating eye that is necessary to minimise the DVD associated hyperdeviation will disrupt vertical orientation (fig l, right). The neutral head position maintained by many patients with DVD may therefore represent a compromise position. To the extent that there is little binocular vision and an asymmetric DVD, however, one might expect the drive for vertical orientation to override, resulting in a head tilt towards the side of the fixing eye (that is, one that is driven by a human dorsal light reflex and non-compensatory for binocular vision).

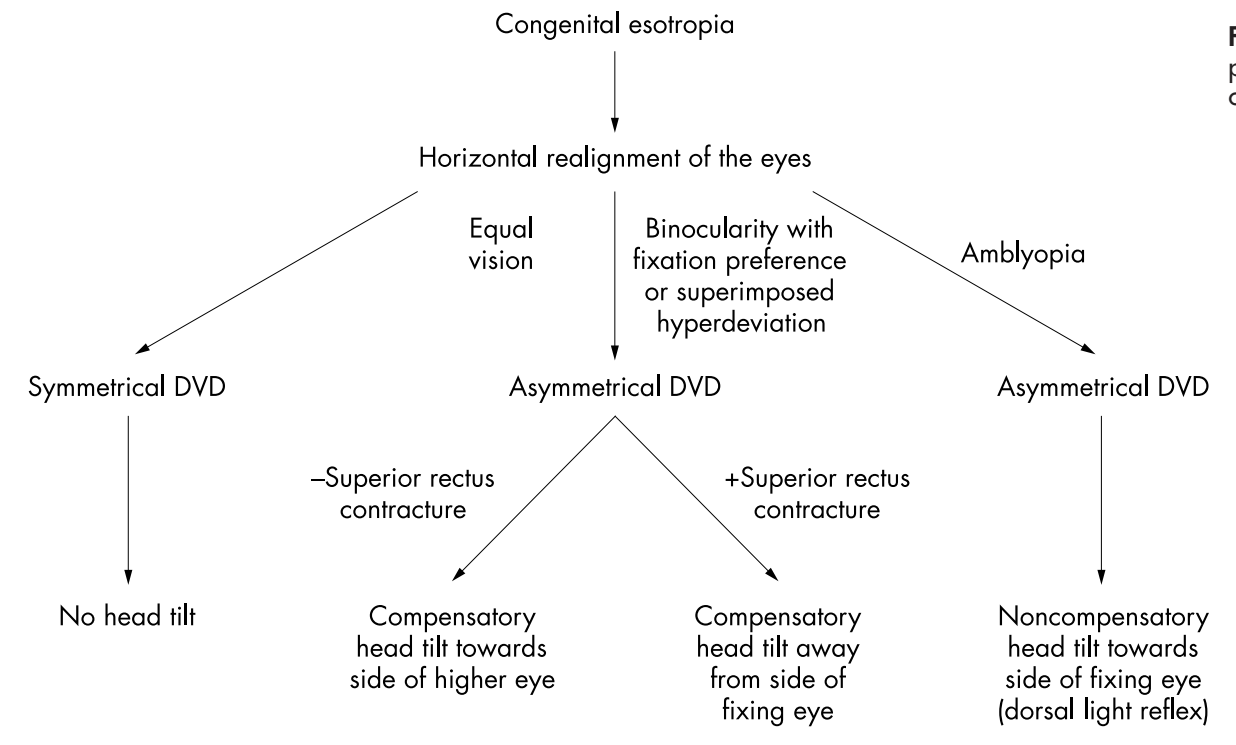

Figure 2 Clinical algorithm depicting postural mechanisms for head tilting in congenital esotropia. 
Alternatively, a strong potential for fusion and stereopsis would cause the drive for binocular vision to override, resulting in a head tilt towards the side of the hyperdeviating eye (which is compensatory for binocular vision). The earlier surgery and better development of stereopsis in group 2 compared with group 1 provides preliminary support for this mechanism.

From a therapeutic perspective, our findings suggest that strabismus surgery to neutralise an asymmetric hyperdeviation should eliminate the compensatory head tilt in group 2 . As a head tilt attributable to a dorsal light reflex does not exist to realign the eyes, surgical treatment of the associated hyperdeviation should not eliminate it. ${ }^{14}$ Prieto-Diaz and Souza-Dias ${ }^{8}$ and von Noorden ${ }^{21}$ have eliminated the head tilt in patients corresponding to group 1 by performing surgery to torsionally rotate the fixing eye in the direction of the head tilt. We believe that torsional rotation of the fixing eye may counterrotate a tilted subjective vertical back to true vertical, thereby eliminating the internal postural drive for the head tilt.

Our study needs to be viewed in terms of its inherent limitations. First, it is a retrospective study, so that all diagnostic tests were not performed on every patient. Ideally, it would be desirable to prospectively assess occlusion of each eye, prismatic neutralisation of the hyperdeviation, Bielschowsky head tilt test, and monocular subjective visual vertical in each patient. Second, our patient numbers for each group were small, so this study should be viewed as a pilot study that requires future confirmation by prospective studies. Third, our results cannot be applied to all patients with congenital esotropia who underwent horizontal strabismus surgery because we excluded patients with primary oblique muscle overaction to eliminate the potentially confounding effects of torsion in a monocularly viewing patient. Finally, while the findings in group 1 of a noncompensatory head tilt, a manifest hyperdeviation of the contralateral eye, and head tilt resolution with patching of the fixing eye but not the hyperdeviating eye (since the hyperdeviating eye is already suppressed) are all consistent with the visuo-vestibular disturbance that would occur with a human dorsal light reflex, definitive confirmation of this mechanism must await further study of the orientation of the subjective vertical and its correlation to the head position in these patients.

In conclusion, an acquired head tilt in the child with surgically corrected congenital esotropia seems to be a postural manifestation of asymmetrical DVD. The head tilt may be the initial sign of DVD, and its underlying mechanism may take years to elucidate. Our findings indicate that a head tilt towards the side of the fixing eye reflects a prenuclear imbalance in central vestibular tone corresponding to a dorsal light reflex, while a head tilt towards the side of the hyperdeviating eye is a compensatory posture to promote vertical binocular alignment. In both groups, the potential role of occlusion therapy in altering the head position needs to be elucidated. When a head tilt persists after occlusion therapy, strabismus surgery to equalise the hyperdeviation in the two eyes should eliminate a compensatory head tilt that is directed towards the side of the higher eye. When a head tilt is directed towards the side of the fixing eye, surgery to torsionally rotate the fixing eye in the direction of the head tilt may have application.

\section{Authors' affiliations}

M C Brodsky, University of Arkansas for Medical Sciences, Little Rock, Arkansas, USA

R Jenkins, Baylor University School of Medicine, Houston, Texas, USA P Nucci, Department of Ophthalmology, Università di Milano, Milan, Italy

Supported in part by a grant from Research to Prevent Blindness Inc.

\section{REFERENCES}

1 Nelson LB, Wagner RS, Simon JW, et al. Congenital Esotropia. Surv Ophthalmol 1987;31:363-83.

2 Von Noorden GK. Binocular Vision and Ocular Motility. Theory and Management of Strabismus. Fifth Edition. St Lovis: Mosby, 1996:312-15.

3 Lang J. Squint dating from birth or with early onset. Transactions of the First International Congress of Orthoptists. London: Henry Kimpton, 1968:231-7.

4 Crone RA. Alternating hyperphoria. Br J Ophthalmol 1954;38:591-604.

5 Betchel RT, Kushner BJ, Morton GV. The relationship between dissociated vertical divergence (DVD) and head tilts. J Pediatr Ophthalmol Strabismus 1996;33:303-6.

6 Santiago AP, Rosenbaum AL. Dissociated vertical deviation and head tilts. JAAPOS 1998;2:5-11.

7 Prieto-Diaz FM. Torticolis en DVD. Presented at Centro Brasileiro de Estrabismo, Brazil: Sao Paolo, April 3-4, 1998

8 Prieto-Diaz J, Souza-Dias C. Strabismus. 4th edn. Boston: ButterworthHeinemann, 1998:203-19.

9 De Decker W, Dannheim-de Decker E. Unbeachtete Zwangshaltungen beim frühkindlichen schielsyndrom. Klin Monatsbl Augenheilkd 1999;215:95-8.

10 Brodsky MC. Dissociated vertical divergence: A righting reflex gone wrong. Arch Ophthalmol 1999;117:1216-22.

11 Holst E von. Bei den Lichtrückenreflex bei Fische. Pubbl Staz Zool (Napoli) 1935; 15:143-8.

12 Graf W, Meyer DL. Central mechanism counteract visually induced tonus asymmetries. J Comp Physiol 1983;150:473-81.

13 Brodsky MC. Vision-dependent tonus mechanisms of torticollis-An evolutionary perspective. Am Orthop J 1999:150-3.

14 Brodsky MC. DVD remains a moving target! J AAPOS 1999;3:325-7.

15 Brodsky MC. Dissociated vertical divergence: Perceptual correlates of the human dorsal light reflex. Arch Ophthalmol 2002;120:1174-8.

16 Pratt-Johnson JA, Tillson G. Management of Strabismus and Amblyopia: A Practical Guide. 2nd edn. New York: Thieme, 2001:95.

17 Guyton DL, Cheeseman EW, Ellis FJ, et al. Dissociated vertical deviation: An exaggerated normal eye movement used to damp cyclovertical nystagmus. Trans Am Ophthalmol Soc 1998;XCVI:389-429.

18 Averbuch-Heller L, Rottach KG, Zivotsky AZ, et al. Torsional eye movements in patients with skew deviation and spasmodic torticollis: Responses to static and dynamic head roll. Neurol 1997;48:506-14.

19 Jampolsky A. Management of vertical strabismus. In: Pediatric Ophthalmology and Strabismus: Transactions of the New Orleans Academy of Ophthalmology. New York: Raven Press, 1986:157-64.

20 Jampolsky A. A new look at the head tilt test. In: Fuchs AF, Brandt Th, Büttner U, Zee DS, eds. Contemporary Ocular Motor and Vestibular Research. A Tribute to David A Robinson. Stuttgart: Springer Verlag, 1994:432-9.

21 von Noorden GK, Jenkins RH, Rosenbaum AL. Horizontal transposition of the vertical rectus muscles for treatment of ocular torticollis. J Ped Ophthalmol Strabis 1993;30:8-14. 


\section{PostScript}

\section{LETTERS}

If you have a burning desire to respond to a paper published in $B J O$, why not make use of our "rapid response" option?

Log on to our web site (www.bjophthalmol. com), find the paper that interests you, and send your response via email by clicking on the "eletters" option in the box at the top right hand corner.

Providing it isn't libellous or obscene, it will be posted within seven days. You can retrieve it by clicking on "read eletters" on our homepage.

The editors will decide as before whether to also publish it in a future paper issue.

\section{Mixed infection (Pseudomonas and coagulase negative staphylococci) microbial keratitis associated with extended wear silicone hydrogel contact lens}

Contact lens induced ulcerative keratitis is a serious complication which can be devastating for the patient if treatment is delayed Extended wear is the commonest cause of microbial keratitis in contact lens wear. ${ }^{1}$ New extended wear silicone hydrogel contact lenses have higher oxygen transmissibility so that they can be worn continuously for 30 days. They can also be used as bandage contact lenses.

The risk of Pseudomonas microbial keratitis with overnight wear is significantly increased by contact lenses with low oxygen transmissibility. ${ }^{2}$ By virtue of high oxygen transmissibility, the silicone hydrogel contact lenses are thought to be associated with low risk of infectious keratitis. ${ }^{34}$ So far only four cases of microbial keratitis have been reported with their use. ${ }^{5}$ In spite of various claims of protection against serious microbial keratitis with pathogens such as $P$ aeruginosa, we have recently come across the first case of Pseudomonas keratitis in a patient wearing silicone hydrogel contact lenses.

\section{Case report}

A 23 year old male patient presented with 1 day history of severe pain, ocular injection, photophobia, and reduced vision of right eye. He was wearing the day and night silicone hydrogel contact lenses, which was replaced

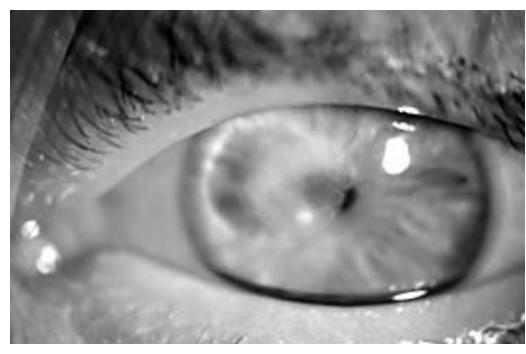

Figure 1 Photograph taken 3 weeks after admission showing central corneal scar. once every 30 days (Ciba vision Focus day and night). He has been wearing these contact lenses for 7 months before the presentation.

Examination revealed a visual acuity of hand movement for the right eye and $6 / 5$ for the left eye. The right eye had a central corneal ulceration of $3 \mathrm{~mm}$ in diameter surrounded by severe oedema and a $1 \mathrm{~mm}$ hypopyon. Cultures grew $P$ aeruginosa and coagulase negative staphylococci both sensitive to ciprofloxacin and gentamicin. Topical ofloxacin and gentamicin were commenced with cyclopentolate. Unpreserved prednisolone eye drops $(0.5 \%)$ were added after I week. Two weeks later, the epithelial defect had resolved leaving behind a central subepithelial corneal scar (fig 1). His vision improved to 6/18 unaided, 6/9 through the pinhole, 1 month after the admission.

\section{Comment}

The major barrier to prescribing a continuous wear contact lens is a perceived danger of microbial keratitis. Many factors are involved in the development of microbial keratitis and these include bacterial adherence to the lens surface, formation of bacterial glycocalyx on the lens, corneal hypoxia, deposits on the lens surface, and the effect of contact lens on closed eye environment. ${ }^{6}$ Silicone hydrogel contact lenses have high oxygen transmissibility and these lenses are colonised by similar numbers and type of micro-organisms compared with HEMA based materials. ${ }^{7}$ A number of studies have shown lower risk of infectious keratitis with new silicone hydrogel contact lenses. ${ }^{3}$

However, the use of silicone hydrogel contact lenses was associated with slightly higher levels of visible deposits, ${ }^{3}$ which may act as a risk factor for bacterial keratitis. As in our case young male patients were also considered a risk factor for contact lens induced microbial keratitis. Our experience suggests that extended wear silicone hydrogel contact lenses are not free of the risk of more serious microbial keratitis caused by $P$ aeruginosa and coagulase negative staphylococci. With increasing popularity among optometrists and the use of silicone hydrogel contact lens as a bandage contact lens, such a serious complication cannot be ignored.

As suggested by other authors, ${ }^{6}$ our experience points towards a multifactorial aetiology for microbial keratitis, rather than just oxygen transmissibility. Further studies are required to find out the safety of the silicone hydrogel contact lenses with regard to development of microbial keratitis.

P Syam, B Hussain, C Hutchinson Department of Ophthalmology, Calderdale Royal Hospital, Salterhebble, Halifax HX3 OPW, UK

Correspondence to: Mr P P Syam, Department of Ophthalmology, Calderdale Royal Hospital, Salterhebble, Halifax HX3 OPW, UK ppsyam@aol.com

Accepted for publication 21 January 2003

\section{References}

1 Dart JKG, Stapleton F, Minassian D. Contact lenses and other risk factors in microbial keratitis. Lancet 1991;338:650-3.
2 Imayasu M, Petroll M, Jester J, et al. The relation between contact lens oxygen transmissibility and binding of pseudomonas aeruginosa to the cornea after overnight wear. Ophthalmology 1994; 101:371-88.

3 Brennan NA, Chantal Coles ML, Comstock TL, et al. A 1-year prospective clinical trial of Balafilcon A (Pure Vision) silicone-hydrogel contact lenses used on a 30-day continuous wear schedule. Ophthalmology 2002;109:1172-7.

4 Nilsson SEG. 7-Day extended wear and 30-day continuous wear of high oxygen transmissibility soft silicone hydrogel contact lenses. A randomised one-year study of 504 patients. CLAO J 2001;27:125-36.

5 Lim L, Loughnan MS, Sullivan L. Microbial keratitis associated with extended wear of silicone hydrogel contact lenses. Br J Ophthalmol 2002;86:355-7.

6 Dart JKG. Contact lens and prosthesis infections. In: Jaeger E, Tasman W, eds. Duane's foundations of clinical ophthalmology, Vol 2. Philadelphia: Lippincott-Raven, 1996:1-30.

7 Keay L, Willcox MDP, Sweeney DF, et al. Bacterial populations on 30-night extended wear silicone hydrogel lenses. CLAO J 2001;27:30-4.

\section{Controlled study of the influence of storage medium type on endothelial assessment during corneal organ culture}

Selection of corneal grafts in eye banks is mainly based on end-of-storage endothelia assessment, which consists of endothelial cell density (ECD) measurement and, to some extent, cell morphometry. Below a certain ECD threshold, generally 2000 cells $/ \mathrm{mm}^{2}$, the cornea is deemed unfit for penetrating keratoplasty. Precise ECD measurement at the end of storage is thus a key issue for eye banks, and also for patients, because it influences the long term survival of the graft. ${ }^{1-3}$

For long term storage in organ culture, the most common method in Europe, ${ }^{4}$ endothelial observation is possible only by transmitted light microscopy. The endothelial cells are exposed to $0.9 \%$ sodium chloride or sometimes to $1.8 \%$ sucrose, which induce a small degree of osmotic cell shrinkage and dilatation of the intercellular spaces thus making individual cells visible. The cells can then be counted manually, through a calibrated reticule or from a photograph; or using an advanced image analysis system. $^{5-7}$ Whichever method of count is used, precision depends primarily on good visualisation of the cell borders. It has long since been shown that, even under experimental conditions of perfect cell membrane visualisation using alizarin red, maximum precision ranges from $+5 \%$ to $-5 \%{ }^{8}$

Two commercial media are authorised by the Agence Française de Sécurité Sanitaire des Produits de Santé. They are very similar in composition, both being based on HEPESbuffered Iscove's Modified Dulbecco's medium containing sodium bicarbonate and $2 \%$ fetal bovine serum, with the same $\mathrm{pH}$ of 7.25 but the osmolality of Inosol (Bausch \& Lomb, Chauvin-Opsia, Labège, France) is only $305 \mathrm{mosmol} / \mathrm{kg}$ (range 295-315) compared with $320 \mathrm{mosmol} / \mathrm{kg}$ (range 300-340) for CorneaPrep/Max (Eurobio, Les Ulis, France). One has nevertheless acquired the reputation of allowing better visualisation of endothelial 
Table 1 Qualitative grading of the endothelial images

\begin{tabular}{lll}
\hline Image quality & Score & Criteria \\
\hline Good & 2 & $\begin{array}{l}\text { Excellent view of cell borders; low background noise; cells visible on over } \\
2 / 3 \text { of image area }\end{array}$ \\
Average & 1 & $\begin{array}{l}\text { Good view of cell borders; moderate background noise; cells visible on } 1 / 3 \\
\text { to } 2 / 3 \text { of image area }\end{array}$ \\
Poor & 0 & $\begin{array}{l}\text { Poor view of cell borders; high background noise; cells visible on less than } \\
1 / 3 \text { of image area }\end{array}$ \\
\hline
\end{tabular}

For the analyses in tri-image mode, overall quality was graded "good" if the three images obtained scores of $2 / 2 / 2$ or $2 / 2 / 1$, "average" if the scores were $2 / 1 / 1,2 / 1 / 0$, or $1 / 1 / 1$, and "poor" if the scores were $1 / 1 / 0,1 / 0 / 0$, or $0 / 0 / 0$. cells and thus facilitating ECD measurement. We therefore compared the quality of endothelial cells visualisation in these two commercial media, using an original image analyser specially designed for the assessment of corneal endothelium by light microscopy.

\section{Methods}

We conducted a randomised prospective study with masked analysis of the results. Donors with history of anterior segment surgery were excluded. After procurement of a pair of corneoscleral discs, one of the corneas (group A) was immersed in Inosol and the other (group B) in Corneaprep/Max for organ culture at $+31{ }^{\circ} \mathrm{C}$. The media were allocated to the right or left cornea according to a randomisation list. Two consecutive endothelial examinations were performed in a similar fashion. The initial examination was done between the first and fifth days after procurement, and the final one two days before cornea delivery.

After the endothelial side was incubated for four minutes in $0.9 \%$ sodium chloride (Aguettant, Lyon, France), it was observed under a direct light microscope (Laborlux, Leica, Wetzlard, Germany) with $\times 10$ original magnification. Ten wide-field $(1250 \times 950 \mu \mathrm{m})$, non-overlapping images of the mosaic, contained within the central $8 \mathrm{~mm}$, were captured by a black and white mono CCD camera. The evaluation was performed by an experienced technician

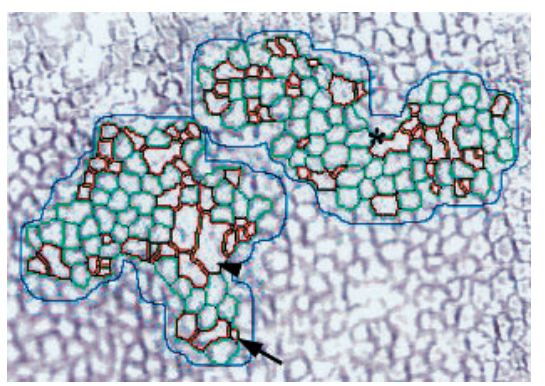

Figure 1 Illustration of the image analysis tools specifically developed for easier touching up of cell borders. Areas outlined in red were either $<150 \mu \mathrm{m}^{2}$ (black arrow), often artefacts like nucleus, debris, or $>1500 \mu \mathrm{m}^{2}$ (arrowhead) or with width greater than twice the length (asterisk), the later two indicating poorly separated cells. These cells, assumed to be poorly recognised, would suggest to the technician the need for contour correction or, where appropriate, validation. To calculate the endothelial cell density in automatic mode, only the green reliably recognised cells were taken into account. masked to storage medium, using a Sambacornée analyser (Samba Technologies, Meylan, France), the commercial version of the "tri-image" analyser prototype developed by our team and described previously. ${ }^{9}{ }^{10} \mathrm{~A}$ fully automatic and a touch up analysis (with user intervention to identify cell boundaries missed or delineated incorrectly in automatic mode) of exactly the same zones of the same three images, were performed on receipt (initial examination) and on delivery (final

The three images selected by the analyser were qualitatively assessed on a scale of three (Table 1) by three independent observers masked to storage medium. Discordant cases were reviewed.

The normality of the data distribution was tested using both the Lilliefors variant of the Kolmogorov-Smirnov test and Shapiro-Wilk normality test, with the cut off for nonnormality set at $\mathrm{p}<0.05$. The quantitative variables (number of cells "reliably recognised", ECD, touch up duration) were compared using a paired $t$ test in the case of normal distribution, and otherwise by a nonparametric test (Wilcoxon). The image quality scores were compared by the $\chi^{2}$ test in a $3 \times 2$ grid. $\mathrm{p}<0.01$ was deemed significant.

\section{Results}

As the study design required inclusion of paired corneas having had two successive analyses, of a series of 77 pairs of corneas procured consecutively, 30 pairs of 13 women $(43 \%)$ and 17 men $(57 \%)$ with a mean age of 69 years (range 30-92) were included in this study. Mean time between death and cornea examination) (Fig 1). procurement was 10 hours (range 0 , for heart beating donors-24).

At the initial examination (Table 2), performed on an average three days (range 1-5) after procurement, image quality was comparable between the two groups. Whichever analysis mode (automatic or touch up) was used, all parameters were comparable between the two media except for the touch up duration which was slightly shorter, on an average by 1 minute (59 seconds, $95 \%$ confidence interval, CI (19-102)), for the corneas in group A. Compared to the automatic analysis, the touch up analysis only slightly decreased the initial ECD value in group A, by 154 cells $/ \mathrm{mm}^{2}$ (95\% CI 36 to 79 ), or $-4.7 \%$ $(\mathrm{p}<0.001)$ and insignificantly in group B, by 101 cells $/ \mathrm{mm}^{2}$ (95\% CI -39 to 240$)$, or $-3.1 \%$ $(\mathrm{p}=0.150)$.

Between the initial and final examination (Table 3), performed on an average 13 days (range 8-22) after procurement, image quality deteriorated markedly in group A $(\mathrm{p}<0.001)$ but remained stable in group $B$ $(\mathrm{p}=0.357)$. At the final examination, group A displayed no "good" images against nearly one in two for group B. Automatic recognition of the cells was thus made much easier in group B, with on an average 238 (46) against only 159 (47) cells in group A $(\mathrm{p}<0.001)$. For group $\mathrm{B}$, the need for touch up was reduced, with a mean time gain of about 3 minutes ( 163 seconds, 95\% CI 116 to $211), \mathrm{p}<0.001)$ and allowing a higher number of cells to be taken into account (456 $(82 \%)$ against $357(72 \%)$ for group A, $\mathrm{p}<0.001)$. In both groups, the touch up analysis reduced considerably the final ECD value compared to the automatic analysis, by 435 cells $/ \mathrm{mm}^{2}$ (95\% CI 317 to 552), or $-13.8 \%(\mathrm{p}<0.001)$ for group $\mathrm{A}$ and by 313 cells $/ \mathrm{mm}^{2}$ (95\% CI 239 to 386$)$, or $-10.3 \%$, $(\mathrm{p}<0.001)$ for group B.

The two media did not differ in terms of preserving endothelial viability: ECD, percentage cell loss, and morphometry (all determined in touch up mode) were comparable between the two media.

\section{Comment}

Our randomised, prospective parallel group study, performed masked with an objective image analysis tool, demonstrates that visualisation of the endothelial mosaic is better after organ culture in CorneaPrep/Max
Table 2 Initial examination of paired corneas stored in medium $A$ and $B$

\begin{tabular}{|c|c|c|c|}
\hline & Group A $(n=30)$ & Group B $(n=30)$ & p Value \\
\hline $\begin{array}{l}\text { Image quality (good/average/ } \\
\text { poor,(\%)) }\end{array}$ & $56 / 27 / 17$ & $43 / 30 / 27$ & 0.526 \\
\hline $\begin{array}{l}\text { Cells well recognised per se, } \\
\text { "automatic" mode (n) }\end{array}$ & $\begin{array}{l}298 \text { (68) } 147-417 / \\
304\end{array}$ & $\begin{array}{l}275(89) 113-417 / \\
284\end{array}$ & 0.058 \\
\hline $\begin{array}{l}\text { ECD, "automatic" mode } \\
\left(\text { cells } / \mathrm{mm}^{2}\right)\end{array}$ & $\begin{array}{l}3267(197) 2779- \\
3672 / 3250\end{array}$ & $\begin{array}{l}3248(305) 2445- \\
3753 / 3249\end{array}$ & 0.770 \\
\hline Cells counted, "touch up" mode (n) & $\begin{array}{l}515 \text { (126) 278-973/ } \\
521\end{array}$ & $\begin{array}{l}540 \text { (259) 314-1777/ } \\
519\end{array}$ & 0.579 \\
\hline ECD, "touch up" mode (cells $/ \mathrm{mm}^{2}$ ) & $\begin{array}{l}3113(341) \text { 2343- } \\
4008 / 3107\end{array}$ & $\begin{array}{l}3147 \text { (391) 2461- } \\
3893 / 3190\end{array}$ & 0.533 \\
\hline $\begin{array}{l}\text { Coefficient of variation of cell area, } \\
\text { "touch up" mode (\%) }\end{array}$ & $\begin{array}{l}29.1(6.1) 21.2- \\
47.2 / 28.1\end{array}$ & $\begin{array}{l}29.1(4.3) 21.7- \\
39.6 / 29.1\end{array}$ & 0.986 \\
\hline Hexagonality, "touch up" mode (\%) & $\begin{array}{l}53.8(9.6) 32.0- \\
75.0 / 53.2\end{array}$ & $\begin{array}{l}51.6(9.0) 34.8- \\
69.6 / 51.9\end{array}$ & 0.212 \\
\hline Touch up duration (seconds) & $\begin{array}{l}345 \text { (131) 129-560/ } \\
334\end{array}$ & $\begin{array}{l}406(166) 171-721 / \\
382\end{array}$ & 0.006 \\
\hline
\end{tabular}

Results were expressed as mean (standard deviation), minimum-maximum/median. The automatic analysis mode provides reliable results compared to the more time consuming touch up one. This allowed, in our eye bank, to decide in a few moments whether to continue with organ culture. 
Table 3 Final examination of paired corneas stored in medium $A$ and $B$

\begin{tabular}{llll}
\hline & Group A (n=30) & Group B (n=30) & p Value \\
\hline Image quality (good/average/poor, (\%)) & $0 / 23 / 77$ & $43 / 43 / 14$ & $<0.001$ \\
Cells well recognised per se, "automatic" & $159(47) 75-248 /$ & $238(46) 151-$ & $<0.001$ \\
mode (n) & 159 & $324 / 238$ & \\
ECD, "automatic" mode (cells/mm²) & $3143(159) 2829-$ & $3029(188) 2650-$ & 0.011 \\
& $3543 / 3105$ & $3451 / 3039$ & \\
Cells counted, "touch up" mode (n) & $357(72) 209-559 /$ & $456(82) 292-$ & $<0.001$ \\
& 339 & $608 / 446$ & \\
ECD, "touch up" mode (cells/mm & $2708(320) 2277-$ & $2716(284) 2259-$ & 0.934 \\
& $3430 / 2704$ & $3579 / 2686$ & \\
Overall cell loss, "touch up" mode (\%) & $12.6(9.3) 7.2-$ & $13.1(8.0) 1.3-$ & 0.79 \\
& $34.1 / 12.2$ & $27.8 / 15.6$ & \\
Coefficient of variation of cell area, "touch & $28.4(3.5) 23.7-$ & $27.2(3.4) 21.7-$ & 0.098 \\
up" mode (\%) & $40.4 / 27.8$ & $34.8 / 27.3$ & \\
Hexagonality, "touch up" mode (\%) & $50.7(9.2) 32.5-$ & $53.0(6.6) 36.2-$ & 0.201 \\
& $72.8 / 49.7$ & $66.6 / 52.6$ & \\
Touch up duration (seconds) & $551(134) 361-$ & $388(120) 226-$ & $<0.001$ \\
& $853 / 521$ & $653 / 362$ & \\
& & &
\end{tabular}

Results were expressed as mean (standard deviation), minimum-maximum/median. The automatic analysis was less relevant at delivery than at receipt (see Table 1), consequently, a touch up analysis should be recommended.

medium than in Inosol. The former facilitates ECD measurement at delivery, the main parameter of corneal quality control. Our study shows that: (1) prolonged storage in CorneaPrep/Max caused no deterioration in image quality, unlike that with Inosol. Better visualisation of cell borders at delivery shortened touch up durations considerably, on average by three minutes per computerised analysis. This point should be particularly relevant for the eye banks which perform endothelial assessment only once, generally after $10-15$ days of organ culture, ${ }^{41}$ (2) At the end of organ culture, the corneas stored in CorneaPrep/Max responded better to osmotic dilation of the intracellular spaces than those stored in Inosol. There was no such discrepancy at the start of storage. We may assume that this difference in behaviour is due to the increased osmolality of CorneaPrep/Max, which is $15 \mathrm{mOsm} / \mathrm{l}$ greater than that of Inosol. During organ culture, the very gradual change of ionic content between the cells and/or of the intercellular junctions may increase water egress from the cells and thus promote dilation of the intercellular spaces in the presence of $0.9 \%$ sodium chloride. However it is likely that over three days these changes did not have time to occur, which would explain the lack of initial differences in image quality. Further histological study could confirm the nature of these changes in the cells and/or their junctions but whatever their nature, they do not affect viability.

In 2001, the twenty one French eye banks stored on average 292 corneas (range 32846). ${ }^{11}$ Because of their small size, the eye banks naturally prefer commercial organ culture media to their own preparations. The two media authorised for use in France are very similar in composition; neither is superior in terms of preserving endothelial viability, as our study confirms. Until now, medium selection was dictated essentially by economic arguments. Our study provides an additional criterion, namely higher quality of mosaic visualisation to justify the choice of medium. It should be possible to extrapolate the beneficial impact of good mosaic visualisation to manual counting, the method still used by most French and European eye banks.

Improving endothelial quality control has become a priority in our eye bank and research laboratory. ${ }^{70}$ Work is under way to determine the ideal composition of organ culture medium that allows the best endothelial visualisation without inducing additional cell loss.

G Thuret, C Manissolle, S Herrag, N Deb, L Campos-Guyotat, P Gain

'Cell survival and adhesion in cancers and grafts,' EA 3063, Faculty of Medicine, University Hospital of Saint-Etienne, France

G Thuret, N Deb, P Gain

Ophthalmology Department, Bellevue Hospital, University Hospital, France

S Acquart

Cornea Bank/French Blood Centre, Saint-Etienne,

France

Correspondence to: Gilles Thuret, Service d'Ophtalmologie, Pavillon 50A, Hôpital Bellevue, CHU Saint-Etienne, 42055 Saint-Etienne Cedex 2 France; gilles.thureł@univ-st-etienne.fr

Presented in part at the 15th annual meeting of the European Eye Bank Association held in Brussels on 17-19 January 2003.

Accepted for publication 26 May 2003

\section{References}

1 Nishimura JK, Hodge DO, Bourne WM. Initial endothelial cell density and chronic endothelial cell loss rate in corneal transplants with late endothelial failure. Ophthalmology 1999; 106:1962-5.

2 Bourne WM. Cellular changes in transplanted human corneas. Cornea 2001;20:560-9.

3 Thuret G, Chiquet C, Bernal F, et al. Prospective randomized clinical and endothelial evaluation of two storage times for corneal donor tissue in organ culture at 31 degrees centigrade. Arch Ophthalmol 2003;121:442-50.

4 EEBA. European Eye Bank Association Directory. Eleventh ed., Amsterdam, 2003.

5 Barisani-Asenbauer T, Baumgartner I, Grabner $G$, et al. Automated digital image analysis of organ culture preserved donor corneas. Ophthalmic Res 1993;25:94-9.

6 Reinhard T, Spelsberg H, Holzwarth D, et al. Wissensbasierte Bildanalyse des Endothels von Hornhauttransplantaten. Klin Monatsbl Augenheilkd 1999;214:407-11.

7 Thuret G, Manissolle C, Acquart S, et al. Is manual counting of corneal endothelial cell density in eyebanks still acceptable? The French experience. Br J Opthalmol 2004 (in press).

8 Sperling S, Gundersen HJ. The precision of unbiased estimates of numerical density of endothelial cells in donor cornea. Acta Ophthalmol (Copenh) 1978;56:793-802.

9 Gain P, Thuret G, Chiquet C, et al. Automated analyser of organ cultured corneal endothelial mosaic. J Fr Ophtalmol 2002;25:462-72.

10 Gain P, Thuret G, Kodjikian L, et al. Automated tri-image analysis of stored corneal endothelium. Br J Ophthalmol 2002;86:801-8.

11 Delbosc B. French directory of corneal storage centres. Ninth ed. Besançon, 2001.

\section{Prospective case control study on genetic assocation of apolipoprotein $\epsilon 2$ with intraocular pressure}

Glaucomas are a leading cause of blindness throughout the world. This group of diseases has a common characteristic: degeneration of the optic nerve that is usually associated with increased intraocular pressure (IOP) Increased IOP is one of the major risk factors for developing glaucomatous damage, whereby the loss of retinal ganglion cells is the typical pathological finding. However, the pathophysiology of pressure induced glaucomatous optic neuropathy remains unclear and is still a matter for debate. Genome scans have been performed to identify the genomic locations of glaucoma susceptibility genes.

Apolipoprotein E (APOE), a lipid transporting protein produced in the liver and brain, is unique among apolipoproteins in that it has particular relevance to nervous tissue. It is involved in the mobilisation and redistribution of cholesterol in repair, growth, and maintenance of myelin and neuronal membranes during development or after injury. Recently it has been shown that the APOE $€ 4$ allele is associated with elevated risk of normal tension glaucoma. ${ }^{2}$ The APOE $\epsilon 2$ allele was shown to be significantly associated with an elevated risk of age related macular degeneration (AMD).

\section{Material and methods}

This prospective case control study included 32 controls (IOP $<22 \mathrm{~mm} \mathrm{Hg}$, normal optic disc, normal visual field), 54 patients with ocular hypertension (OHT, IOP > $11 \mathrm{~mm} \mathrm{Hg}$, normal optic disc, normal visual field), 96 patients with primary open angle glaucoma (POAG, 55 patients with preperimetric open angle glaucoma (pre-OAG, IOP $>21 \mathrm{~mm} \mathrm{Hg}$, glaucomatous optic disc, normal visual field), and 41 patients with perimetric open angle glaucoma (OAG, IOP $>21 \mathrm{~mm} \mathrm{Hg}$, glaucomatous optic disc, visual field defects). All individuals included in the study were unrelated, white, and had open anterior chamber angles, clear optic media, and a visual acuity of $20 / 25$ or better. Exclusion criteria were all eye diseases other than glaucoma, diabetes mellitus, myopic refractive error exceeding -8 diopters, and visual acuity less than 0.7 .

The study followed the tenets of the declaration of Helsinki for research involving human subjects and informed consent was obtained from all participants.

All control subjects and patients were thoroughly examined by clinical biomicroscopy including slit lamp inspection, gonioscopy and ophthalmoscopy, applanation 
Table 1 Distribution of APOE genotypes and allele frequency

\begin{tabular}{|c|c|c|c|c|c|}
\hline & \multicolumn{5}{|c|}{ Frequency in APOE characteristic (n (female) mean age in years) } \\
\hline & $\begin{array}{l}\text { Normals } 32 \\
\text { (17) } 54.8\end{array}$ & $\begin{array}{l}\text { OHT } 54 \text { (27) } \\
53.3\end{array}$ & $\begin{array}{l}\text { Pre-OWG } 55 \\
\text { (25) } 54.4\end{array}$ & $\begin{array}{l}\text { OWG } 41 \\
\text { (20) } 56.3\end{array}$ & $\Sigma$ \\
\hline \multicolumn{6}{|c|}{ Genotype } \\
\hline$\in 2 \in 2$ & 0 & $1(0)$ & 0 & 0 & 1 (0) 63.0 \\
\hline$\epsilon 2 € 3$ & $3(2)$ & $11(5)$ & $12(7)$ & $6(2)$ & 32 (16) 56.0 \\
\hline$\epsilon 2 \in 4$ & $6(5)$ & 0 & 1 (0) & 0 & 7 (5) 53.4 \\
\hline$€ 3 € 3$ & $14(6)$ & 32 (17) & $24(12)$ & 27 (15) & $97(50) 54.5$ \\
\hline$€ 3 € 4$ & $9(6)$ & $9(5)$ & $18(6)$ & $8(3)$ & 44 (18) 53.8 \\
\hline$\in 4 \in 4$ & 0 & $1(0)$ & 0 & 0 & 1 (0) 43.0 \\
\hline \multicolumn{6}{|c|}{ Allele frequency } \\
\hline$\epsilon 2$ & 9 & 13 & 13 & 6 & 41 \\
\hline$\epsilon 3$ & 40 & 84 & 78 & 68 & 270 \\
\hline$€ 4$ & 15 & 11 & 19 & 8 & 53 \\
\hline
\end{tabular}

tonometry, perimetry (Octopus Gl program, 3 phases), and pachymetry (Tomey AL-1100). In addition, a 24 hours IOP curve with measurements at $7 \mathrm{am}, 12 \mathrm{am}, 5 \mathrm{pm}, 9 \mathrm{pm}$, $12 \mathrm{pm}, 7$ am was measured in all patients.

For a classification of study groups the $15^{\circ}$ colour stereo photographs were evaluated qualitatively by two observers. Criteria for the diagnosis in all glaucomas were increased IOP and glaucomatous changes of the optic nerve head, including abnormally small neuroretinal rim area in relation to the optic disc size, abnormal neuroretinal rim shape, cup:disc ratios being higher vertically compared with horizontally, and localised or diffuse loss of retinal nerve fibre layer.

All subjects were familiar with visual field testing. Subjects with a higher than $12 \%$ rate of false-positive or false-negative responses were excluded. A "perimetric" glaucomatous visual field was defined as an Octopus Gl field with (a) at least three adjacent test points having a deviation of equal to or greater than $5 \mathrm{~dB}$ and with one test point with a deviation greater than $10 \mathrm{~dB}$ lower

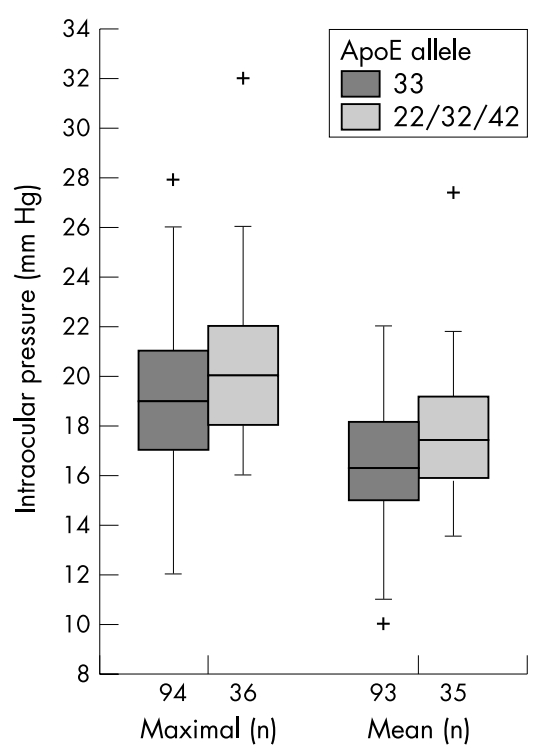

Figure 1 Maximal and mean IOP of the 24 hour diurnal curve of 130 individuals (normal controls, OHT, pre-OAG, and OAG; + cases with more than 1.5 box lengths from the upper or lower edge of the box. The box length is the interquartile range. than normal; (b) at least two adjacent test points with a deviation equal to or greater than $10 \mathrm{~dB}$; (c) at least three adjacent test points with a deviation equal to or greater than $5 \mathrm{~dB}$ abutting the nasal horizontal meridian, or (d) a mean visual field defect of more than $2.6 \mathrm{~dB}$.

For APOE genotyping, genomic DNA was extracted from anticoagulated blood after isolation of peripheral lymphocytes following the "salting out" method. The APOE gene shows a polymorphism with three alleles $(\epsilon 2$, $\epsilon 3, \epsilon 4)$ (table 1). As allele $\epsilon 3$ is considered to be the ancestral allele, and $\epsilon 2$ and $\epsilon 4$ are considered as variants on the basis of single point mutations, the $\epsilon 3 \in 3$ genotype was used as reference.

\section{Results}

The mean (SD) IOP of the $24 \mathrm{~h}$ diurnal curve was significantly higher in subjects with the $\epsilon 2$ allele (independent samples $t$ test, $t=-2.57, \quad \mathrm{p}=0.011, \quad 17.7 \quad(2.7) \quad v \quad 16.4$ (2.4) $\mathrm{mm} \mathrm{Hg}$ ) (fig l). The maximum and minimum IOP were also increased or decreased, but not significantly (MannWhitney $\mathrm{U}, \mathrm{p}=0.052,20.5 \quad(3.2) \quad v \quad 19.1$ (3.0) $\mathrm{mm} \mathrm{Hg}$, respectively. $\mathrm{p}=0.178,14.8$ (2.7) $v 14.0(2.5) \mathrm{mm} \mathrm{Hg}$ ). This was approximately continuous in the different study groups (normals, OHT, pre-OAG, and OAG); however it was only significant in the normal subjects for mean $(t=-2.183, \mathrm{p}=0.043,18.0$ (4.9) $v 14.3(2.5) \mathrm{mm} \mathrm{Hg}$ ) and minimum IOP $(\mathrm{U}=15, \quad \mathrm{p}=0.031, \quad 15.0 \quad$ (3.9) $\quad v \quad 11.7$ (2.5) $\mathrm{mm} \mathrm{Hg}$ ). The central corneal thickness was not different between the subjects with the $\epsilon 2$ allele and the reference group with $\epsilon 3 \epsilon 3$ genotype $(t=-0.035, \mathrm{p}=0.973,587$ (33) $v 586(51) \mu \mathrm{m})$.

\section{Discussion}

The results of this study show a significant association between the level of IOP and the APO $\epsilon 2$ allele. This may be supported by the recent findings that the APO $€ 4$ allele is associated with higher risk for glaucomatous changes that are not related to increased $\mathrm{IOP}^{2}{ }^{2}$

It is not yet obvious how the APOE alleles may be a source of genetic risk for glaucoma and increased IOP. It will be intriguing to investigate whether there is increased expression of APOE in trabecular meshwork in glaucoma or an isoform dependent expression in different types of glaucoma. A possible role for ApoE promoter single nucleotide polymorphisms as modifiers of the POAG phenotype has been hypothesised. ${ }^{4}$
To conclude, we have shown a significant association between APOE and glaucoma and IOP. Quite recently it was argued that an IOP reduction of $1 \mathrm{~mm} \mathrm{Hg}$ from baseline will decrease the risk of progression by about $10 \% .^{5}$ Although in need of confirmation, our data emphasise the role of APOE in regulation of IOP and may indicate that we have identified a susceptibility gene for glaucoma.

As future perspective for the APOE alleles, analysis of a larger number of glaucoma patients-taking into account family history, age, and sex-will provide more detailed insight.

A Jünemann, N Wakili, C Mardin, G O H Naumann

Department of Ophthalmology, Friedrich-AlexanderUniversity, Erlangen-Nuremberg, Germany

S Bleich, K Henkel, G Beck, J Kornhuber Department of Psychiatry and Psychotherapy, Friedrich-Alexander-University, Erlangen-Nuremberg, Germany

U Reulbach

Department of Medical Informatics, Biometry and Epidemiology, Friedrich-Alexander-University, Erlangen-Nuremberg, Germany

B Rautenstrauss, A Reis Department of Human Genetics, Friedrich-AlexanderUniversity, Erlangen-Nuremberg, Germany

Correspondence to: Dr A Jüneman, Department of Ophthalmology, Friedrich-Alexander-University, Erlangen-Nuremberg, Schwabachanlage 6 Erlangen, Germany; anselm.juenemann@ augen.imed.uni-erlangen

Accepted for publication 7 June 2003

\section{References}

1 Wiggs JL, Allingham RR, Hossain A, et al. Glaucoma-wide scan for adult onset primary open angle glaucoma. Hum Mol Genet 2000;9:1109-17.

2 Vickers JC, Craig JE, Stankovich J, et al. The apolipoprotein 4 gene is associated with elevated risk of normal tension glaucoma. Mol Vis 2002:8:389-93.

3 Klaver CCW, Kliffen M, Duijn CM, et al. Genetic association of apolipoprotein $\mathrm{E}$ with age-related macular degeneration. Am J Human Genet 1998;63:200-6

4 Copin B, Brezin AP, Valtot F, et al. Apolipoprotein E-promoter single-nucleotide polymorphisms affect the phenotype of primary open-angle glaucoma and demonstrate interaction with the myocilin gene. Am J Hum Genet 2002;70:1575-81.

5 Leske MC, Heij A, Hussein M, et al. Factors for glaucoma progression and the effect of treatment: the early manifest glaucoma trial. Arch Ophthalmol 2003;121:48-56.

\section{The safety of anterior chamber paracentesis in patients with uveitis}

Anterior chamber (AC) paracentesis is a valuable procedure in the management of uveitis, particularly in diagnosing infective causes. ${ }^{12}$ It may also be used therapeutically to lower intraocular pressure, ${ }^{3}$ and it provides samples for clinical research. Nevertheless, there have been isolated reports of $\mathrm{AC}$ paracentesis related serious complications, including endophthalmitis and corneal abscess. ${ }^{45}$ As the risk of trauma to the iris and lens are also major concerns, AC paracentesis is often used with reluctance. 
Although there are many studies on the analysis of aqueous humour obtained from AC paracentesis, our literature search showed only one publication on the safety of AC paracentesis. ${ }^{6}$

The purpose of this study was to describe a method of AC paracentesis that can be easily performed as an outpatient procedure with the patient sitting at the slit lamp.

\section{Methods and results}

A total of 70 patients (41 male, 29 female) aged 18-83 years (median 39 years) with various types of active uveitis attending the Birmingham and Midland Eye Centre underwent AC paracentesis. Fourteen paracenteses were performed for diagnostic purposes while the remainder for experimental analysis as part of another study. Patients with dilated and undilated pupils were included. Local research ethics committee approval and informed consent was obtained.

Benoxinate $0.4 \%$ eye drops (minims) were instilled three times over a 3 minute period, followed by instillation of betadine $5 \%$ antiseptic solution that had been drawn up into the empty benoxinate minim container. The patient was positioned at the slit lamp, the upper lid and eyelashes held out of the way by an assistant. No lid speculum was required.

Of the 70 paracenteses, 48 were performed using a 27 gauge needle attached to an insulin syringe, while the remaining 22 were performed using an aqueous pipette. Where a 27 gauge needle was used, this was inserted at the paralimbal clear cornea in a plane above and parallel to the iris with the bevel of the needle facing forward until the whole bevel penetrated the cornea (fig 1). Under direct vision, the sampler pulled the plunger of the syringe to aspirate the aqueous. The aqueous pipette (Visitec, Sarasota, FL, USA designed by O'Rourke) consists of a short 30 gauge needle mounted inside plastic tubing, which in turn is connected to a soft polyethylene suction-infusion bulb. The bulb was squeezed to create a vacuum and the needle inserted at the limbus as described above (fig 2). When pressure on the bulb was released, aqueous spontaneously filled the pipette. Using either method, the eye can be fixed with a pair of forceps at the opposite limbus, if necessary. After sampling an antibiotic drop was prescribed for three days. The whole procedure takes less than five minutes. All patients were re-examined 20 minutes after the procedure and 1-2 weeks later.

Two patients had an air bubble inadvertently injected into the AC. The visual acuity

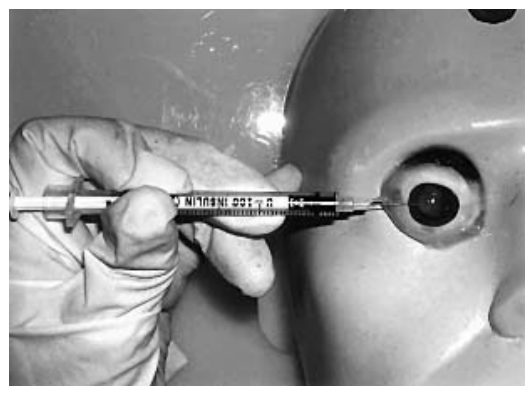

Figure 1 Anterior chamber paracentesis with 27 gauge needle (pig's eye used for demonstration). returned to normal at review in both cases. One patient developed an acute allergic conjunctivitis to betadine, which settled after treatment with prednisolone $0.5 \%$ eye drops. None of the 70 patients developed detectable corneal damage, lens changes, or endophthalmitis.

\section{Comment}

Various methods for performing AC paracentesis have been described ${ }^{6-9}$ However, our literature search only identified one systematic report investigating the safety of $\mathrm{AC}$ paracentesis. ${ }^{6}$ This technique required the patient to lie supine under the microscope, needed insertion of a lid speculum and preincision of the cornea with a $15^{\circ}$ micro sharp blade, and the aqueous was aspirated using a 27 gauge needle on a tuberculin syringe. No serious complications were reported in 361 uveitis patients. A small hyphaema occurred in 5/72 (6.9\%) patients examined 30 minutes after the paracentesis. The method described by O'Rouke using the aqueous pipette ${ }^{7}$ is relatively new and no systematic analysis of its safety profile has been published. Other methods for AC paracentesis include not using a syringe ${ }^{8}$ or a syringe with the plunger removed, ${ }^{9}$ thus avoiding any potential complications associated with aspirating with a plunger, but collecting the aqueous specimen may be technically difficult.

The cases of inadvertent injection of an air bubble into the AC both occurred using the aqueous pipette and was most likely caused by air trapped inside the bulb prior to inserting the pipette into the AC. We recommend ensuring the bulb is thoroughly depressed to evacuate all air before inserting the needle into the eye. Pressure on the bulb must also be maintained while the needle is being inserted, to avoid air entry.

Our study showed that performing AC paracentesis with the patient sitting at the slit lamp is safe using either the 27 gauge needle or the aqueous pipette. Preincision with a sharp blade and the use of a lid speculum is unnecessary.

\section{Acknowledgements}

We are grateful to Robert Harvey and Salman Mirza for their help in this study.

C M G Cheung, O M Durrani, P I Murray Birmingham and Midland Eye Centre, Sandwell and West Birmingham Hospitals NHS Trust, City Hospital, Birmingham, UK

Correspondence to: Professor P I Murray, Academic Unit of Ophthalmology, Division of Immunity and Infection, The University of Birmingham, Birmingham

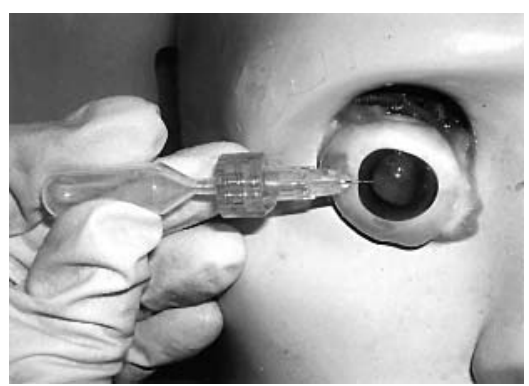

Figure 2 Anterior chamber paracentesis with aqueous pipette (pig's eye used for demonstration). and Midland Eye Centre, Sandwell and West Birmingham Hospitals NHS Trust, City Hospital, Dudley Road, Birmingham B18 7QU,

P.I.Murray@bham.ac.uk

Accepted for publication 10 June 2003

\section{References}

1 Boer de JH, Luyendiik L, Rothova A, et al. Analysis of ocular fluids for local antibody production in uveitis. Br J Ophthalmol 1995;79:610-6.

2 Boer de JH, Verhagen C, Bruinenberg M, et al. Serological and polymerase chain reaction analysis of intraocular fluids in the diagnosis of infectious uveitis. Am J Ophthalmol 1996; 121:650-8

3 Carnahan MC, Platt LW. Serial paracenteses in the management of acute elevations of intraocular pressure. Ophthalmology 2002;109:1604-6.

4 Helbig H, Noske W, Kleineidam M, et al. Bacterial endophthalmitis after anterior chamber paracentesis. Br J Ophthalmol 1995:79:866.

5 Azuara-Blanco A, Katz $\sqcup$. Infectious keratitis in a paracentesis tract. Ophthalmic Surg Lasers 1997; 28:332-3

6 Van der Lelii A, Rothova A. Diagnostic anterior chamber paracentesis in uveitis: a safe procedure? $\mathrm{Br} J$ Ophthalmol 1997;81:976-9.

7 O'Rourke J, Taylor DM, McDonald P, et al. An aqueous paracentesis pipet. Ophthalmic Surg 1991;22:166-7.

8 May DR, Noll FG. An improved approach to aqueous paracentesis. Ophthalmic Surg 1988;19:821-2.

9 Grewal SPS. A technique for paracentesis. Ophthalmic Surg 1989;20:525.

\section{Rapid recovery of night blindness due to obesity surgery after vitamin A repletion therapy}

Night blindness is the most common and earliest symptom of vitamin A deficiency. The latter can be caused by general malnutrition, malabsorption of vitamin A, or impaired vitamin A metabolism due to liver disease. ${ }^{2}$

Several surgical methods are currently used for the treatment of obesity. In the Scopinaro procedure, a biliopancreatic bypass is combined with a bypass of part of the small bowel, thus promoting intestinal malabsorption.

The fat soluble vitamin A can exist as retinol, its ester, and retinoic acid. It has several roles in ocular metabolism: it is essential for corneal and conjunctival epithelial cell RNA and glycoprotein synthesis, while retinal is the crucial chromophore which combines with both rod and cone opsins to form rhodopsin and activated cone opsins, which are essential for phototransduction.

\section{Case report}

A 39 year old man presented with a 6 month history of night blindness, progressing more rapidly in the past 2 weeks. Three years before he had undergone a partial gastrectomy and biliopancreatic derivation for morbid obesity (Scopinaro procedure). His mean body mass index (BMI) decreased from $50 \mathrm{~kg} / \mathrm{m}^{2}$ to $31 \mathrm{~kg} / \mathrm{m}^{2} 3$ years later.

At presentation, visual acuity was $6 / 5$ in both eyes with a spherical correction of +0.75 dioptres. Slit lamp examination and funduscopy were unremarkable in both eyes. Concentric narrowing in both eyes could be seen on Goldmann visual field (VF) analysis (fig lA). Goldmann-Weekers dark adaptometry (DA) 
showed a considerable decrease in sensitivity (fig 1B). Electro-oculography was subnormal before therapy with a light ${ }_{\text {Peak }} /$ dark $_{\text {Trough }}$ ratio of $166 \%$ for the right eye and $146 \%$ for the left eye (normal ratio >180\%). ISCEV standard electroretinography ${ }^{5}$ showed only minimal residual scotopic responses in both eyes. The SRC of vitamin A before therapy was $14 \mu \mathrm{g} / \mathrm{dl}$ (normal range $30-80)$. Vitamin E levels $(0.49 \mathrm{mg} / \mathrm{dl}$; normal 0.5-1.8) and total protein levels were slightly subnormal. Vitamin B and vitamin D levels were normal.

Our patient was given 60000 IU retinol/ day and vitamin E $140 \mathrm{mg} /$ day (Rovigon, Roche).

After only 3 days, partial normalisation of Goldmann VFs occurred. After 3 days of vitamin A supplementation, scotopic ERG responses had already improved to one third of normal (fig 2). Subjectively, the patient reported a "sudden visual recovery" 3 days after initiation of therapy.

After 5 days of therapy the EOG $\mathrm{L}_{\mathrm{p}} / \mathrm{D}_{\mathrm{t}}$ ratio returned to near normal.

Ten days after initiation of treatment, all ERG parameters returned to normal (fig 2). Complete normalisation of DA was also seen (fig lB).

From day 36 Goldmann visual fields were considered to be normal. The ERG (fig 2) and EOG had completely normalised by then.

After 135 days of repletion therapy SRC of vitamin A was still only $26 \mu \mathrm{g} / \mathrm{dl}$, while vitamin $\mathrm{E}$ levels returned to normal $(0.6 \mathrm{mg} / \mathrm{dl})$. Treatment was maintained.

\section{Comment}

Normal biliary secretion, fat absorption, dietary protein intake, and the presence of zinc are necessary for fat soluble vitamin absorption. ${ }^{6}$

Vitamin A has a major role in photoreceptor function because it combines, in the form of its 11-cis isomer, with photoreceptor opsins to form rhodopsin and activated cone opsins.

At presentation, the ERG in our patient showed a considerable decrease in rod and, to a lesser extent, in cone function.

After 3 days of vitamin A repletion a significant improvement in the scotopic responses was noted. All ERG responses normalised completely after only 10 days of therapy. This rapid recovery of all electrophysiological and clinical parameters indicates that vitamin A deficiency was still in the earlier stages. The lag between obesity surgery and symptoms can be attributed to the presence of considerable liver stores of vitamin A when surgery was performed.

Our patient was repleted with 15 times the recommended daily allowance (RDA) of retinol (RDA of retinol 4000 IU/day) and 12 times that of vitamin E (RDA of vitamin E $12 \mathrm{IU} /$ day). Interestingly, vitamin $\mathrm{E}$ deficiency seems to decrease the amount of vitamin A which can be stored in the retina. ${ }^{7}$ Long term vitamin replacement therapy is essential after biliopancreatic derivation surgery of the Scopinaro type.

Only a limited number of reports have described cases of vitamin A deficiency following bowel surgery for obesity.

In 1999 Smets et al described a case of night blindness and optic neuropathy after biliopancreatic bypass with normalisation of all electrophysiological parameters when retested after 10 months.
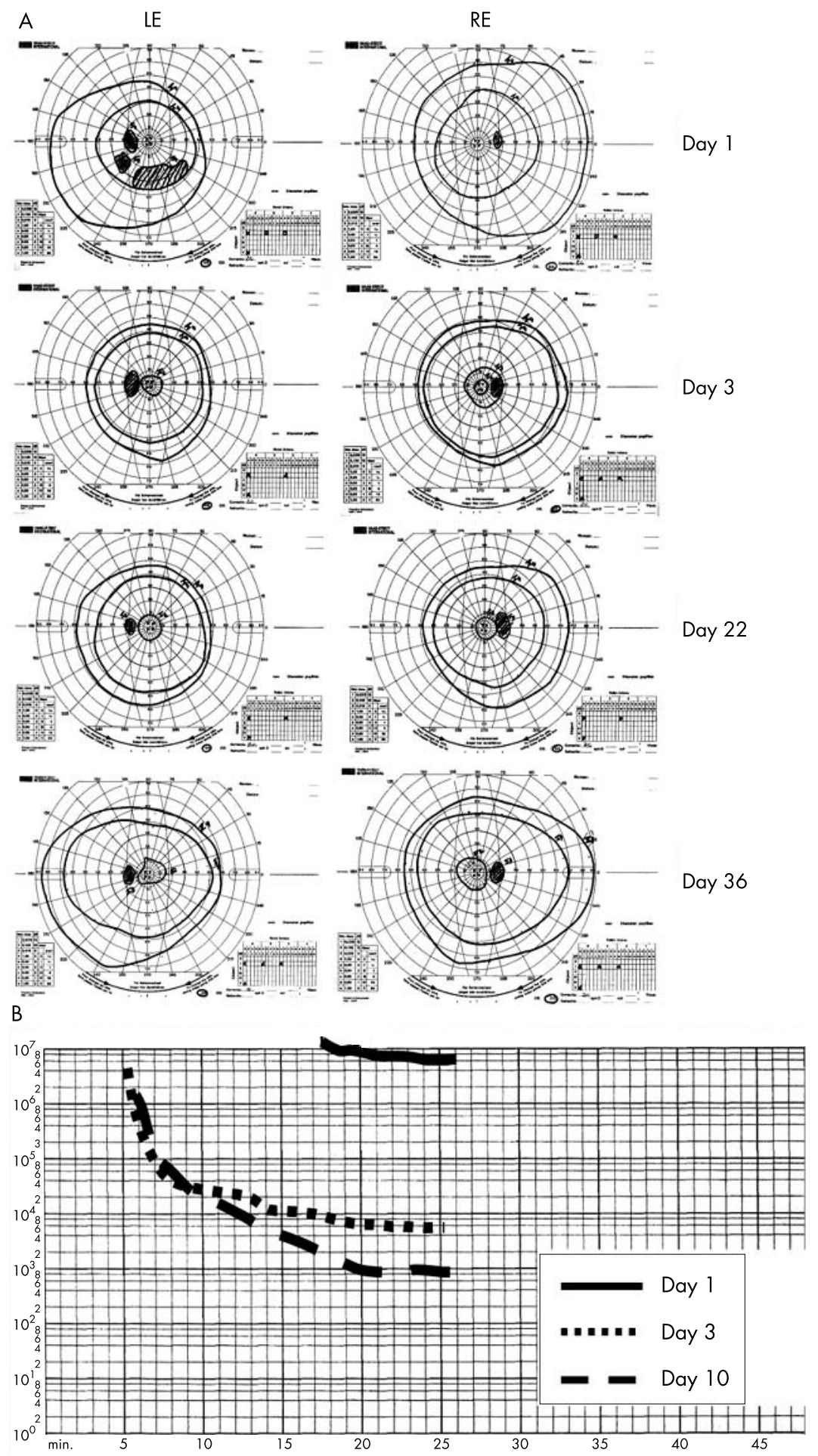

Figure 1 (A) Goldmann visual field analysis; although peripheral limits as tested with object $\mathrm{V} 4$ of Goldmann remained normal, only test object 14 was perceived more centrally, indicating loss of retinal sensitivity. On day 3 of therapy, the patient could already perceive test object I2 and I3 illustrating partial normalisation, while, unexpectedly, peripheral limits were more constricted. From day 36 Goldmann visual fields were considered to be normal. (B) Goldmann-Weekers dark adaptometry (DA) showed considerable decrease in sensitivity before repletion therapy; day 1 is at presentation, before treatment; considerable improvement seen on day 3 , with complete normalisation on day 10.

No cone dysfunction was reported, despite SRC well below those in our patient. $^{8}$
In all reports of vitamin A deficiency to date, the rod involvement is seen earlier and is more extensive than cone 

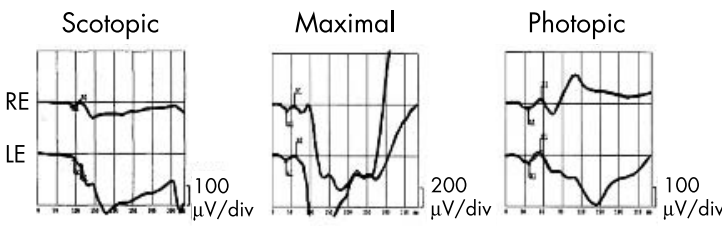

\section{$30 \mathrm{~Hz}$ Flicker}
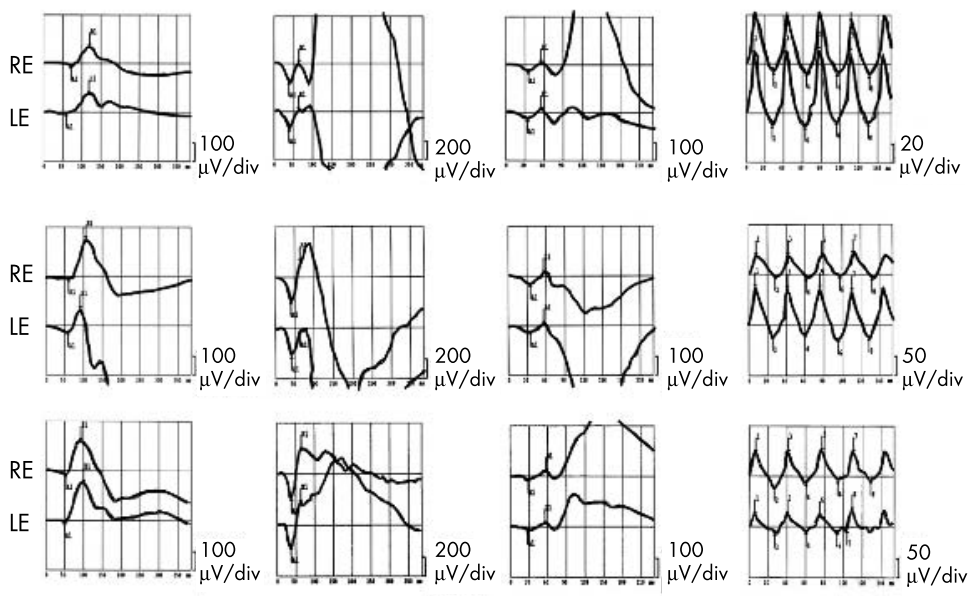

Day 10
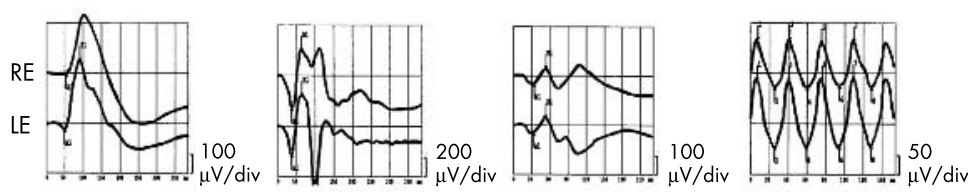

Day 22
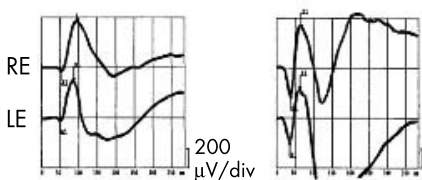

200
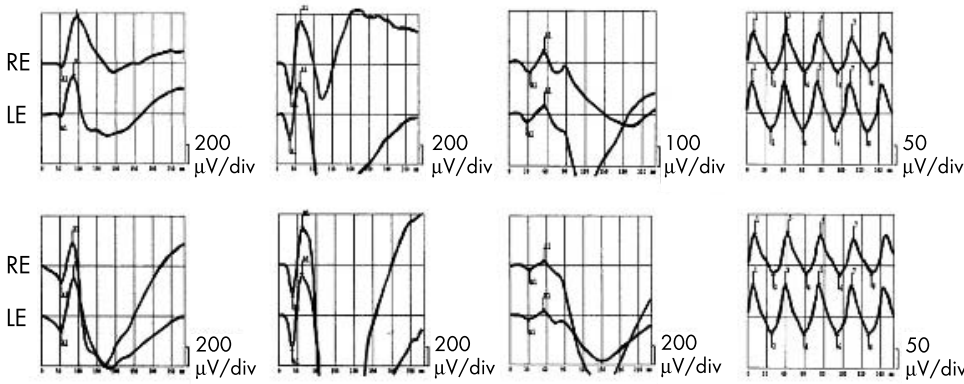

Day 72
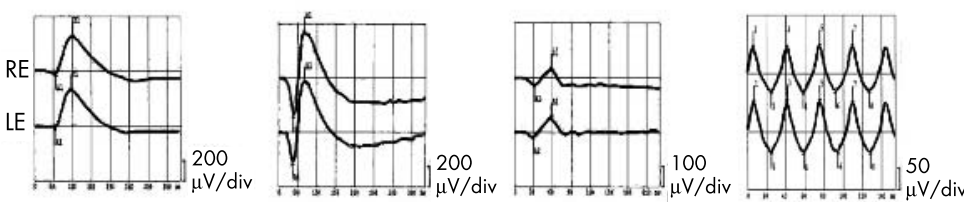

Normal

Control

Figure 2 ERGs on day 1 (before treatment) and subsequent days as indicated; normal control for comparison at bottom. At presentation, only minimal residual scotopic responses were seen in both eyes. Amplitudes of oscillary potentials were only residual. Responses to a single bright white flash in the dark adapted eye were minimal, with amplitudes of approximately one quarter of normal for $a$-wave and $1 / 13$ for $b$-wave in both eyes. Single flash cone responses still had normal a-waves, while b-waves were two thirds of normal in both eyes. The $30 \mathrm{~Hz}$ flicker responses were four fifths of normal in right eye and normal in left eye. After 3 days of vitamin A supplementation, scotopic responses improved to one third of normal in both eyes. Ten days after initiation of treatment, all ERG parameters were within normal limits, although amplitudes still increased up to day 22.

involvement. ${ }^{9}$ The reasons for this remain obscure, although rods are known to be more dependent on availability of vitamin A from the retinal pigment epithelium. ${ }^{10}$

In conclusion, our case proves that malabsorption caused by biliopancreatic derivation surgery of the Scopinaro type can induce vitamin A deficiency with progressive rod-cone dysfunction, as well as deficiencies of all fat soluble vitamins and low plasma proteins.

In the early stages of vitamin A deficiency, recovery of visual function rapidly follows after oral repletion therapy, and can be nearly complete 1 week after initiation of such therapy.

Department of Ophthalmology and Centre for Medical Genetics, Ghent University Hospital, Ghent, Belgium
Y Spits, J-J De Laey, B P Leroy
Correspondence to: B P Leroy, Department of Ophthalmology and Centre for Medical Genetics, Ghent University Hospital, De Pintelaan 185, Ghent, Belgium; bart.leroy@ugent.be

Accepted for publication 15 June 2003

\section{References}

1 Harris EW, Loewenstein Jl, Azar D. Vitamin A deficiency and its effects on the eye. Int Ophthalmol Clin 1998;38:155-61.

2 Groff JL, Gropper SS, Hunt S. Advanced nutrition and human metabolism. St Paul, MN: West Publishing Company, 1995:295-7.

3 Scopinaro N, Gianetta E, Civalleri D. Two years of clinical experience with biliopancreatic bypass for obesity. Am J Clin Nutr 1980;33:506.

4 Smith J, Steinemann TL. Vitamin A deficiency and the eye. Int Ophthalmol Clin 2000;40:83-91.

5 Marmor MF, Zrenner E. Standard for clinical electroretinography (1999 update). Doc Ophthalmol 1998:97:143-56.

6 Tsinopoulos I, Nousia-Arvanitakis S, GalliTsinopoulou A, et al. Role of electroretinography in the assessment of retinal function as an indicator of vitamin A status. Doc Ophthalmol 2000;101:211-21.

7 Robison WG, Kuwabara T, Bieri JG. Vitamin E deficiency and the retina: photoreceptor and pigment epithelial changes. Invest Ophthalmol Vis Sci 1979;18:683-90.

8 Smets RM, Waeben M. Unusual combination of night blindness and optic neuropathy after biliopancreatic bypass. Bull Soc Belge Ophtalmol 1999;271:93-6.

9 Scholl HP, Zrenner E. Electrophysiology in the investigation of acquired retinal disorders. Surv Ophthalmol 2000;45:29-47.

10 Bridges CDB. Retinoids in photosensitive systems. In: Sporn MB, Roberts AB, Goodman DS, eds. The retinoids. New York: Academic Press, 1984:125-76.

Incision-less frontalis suspension

Frontalis suspensions with alloplastic slings are well established. ${ }^{12}$ The thick eyebrow skin of infants is prone to scar formation. Forehead scars caused by frontalis suspension procedures can be problematic. We describe a technique of congenital ptosis surgery that avoids eyebrow incisions.

\section{Surgical technique}

This new procedure utilises a Nylon monofilament suture for frontalis suspension. The Nylon suture is passed in a circlage fashion via puncture wounds without making eyebrow incisions. Two puncture sites, approximately $10 \mathrm{~mm}$ apart, are marked $3 \mathrm{~mm}$ above the lash line centred over the area of desired maximal eyelid elevation. Another two puncture sites are marked above the eyebrow approximately in line with the lateral and medial canthi. The path of the circlage is marked out by joining the marked puncture sites. The eyelid and eyebrow are infiltrated with local anaesthetic with adrenaline (epinephrine)

A Keith needle is dual threaded with a $4 / 0$ Nylon and a 4/0 Vicryl suture. It is then passed from one eyelid puncture site towards the corresponding eyebrow exit site in a suborbicularis plane (fig 1, top left) with the globe protected by a lid guide. From this site, the needle is passed through the needle track to the adjacent eyebrow puncture site (fig l, top right) and then down towards the remaining eyelid puncture site. At this point in the procedure, the ends of the Nylon and Vicryl sutures emerge through the two eyelid puncture sites. The two ends of the Vicryl 
suture are then manoeuvred in a sawing fashion to create friction to release skin dimpling at the eyebrow exit sites (fig 1, bottom left). The Vicryl suture is then removed and the Nylon suture needle $(\mathrm{SH}$ needle) is passed from one eyelid puncture site to another via a deep, partial thickness tarsal passage with the eyelid everted to ensure no full thickness penetration (fig l, bottom right). The two ends of the Nylon suture, exiting at one eyelid puncture site, are tied and the tension adjusted to achieve the desired lid elevation and contour. Occasionally, peaking of the eyelid occurs and can be managed by slightly enlarging the puncture site at the tight suture end with a Westcott scissors and gentle spreading to undermine the soft tissues around the suture. This undermining action helps to release the suture tension on the puncture site to smoothen out the lid contour but should be done carefully to avoid cutting the suture. The puncture sites usually do not require closure.
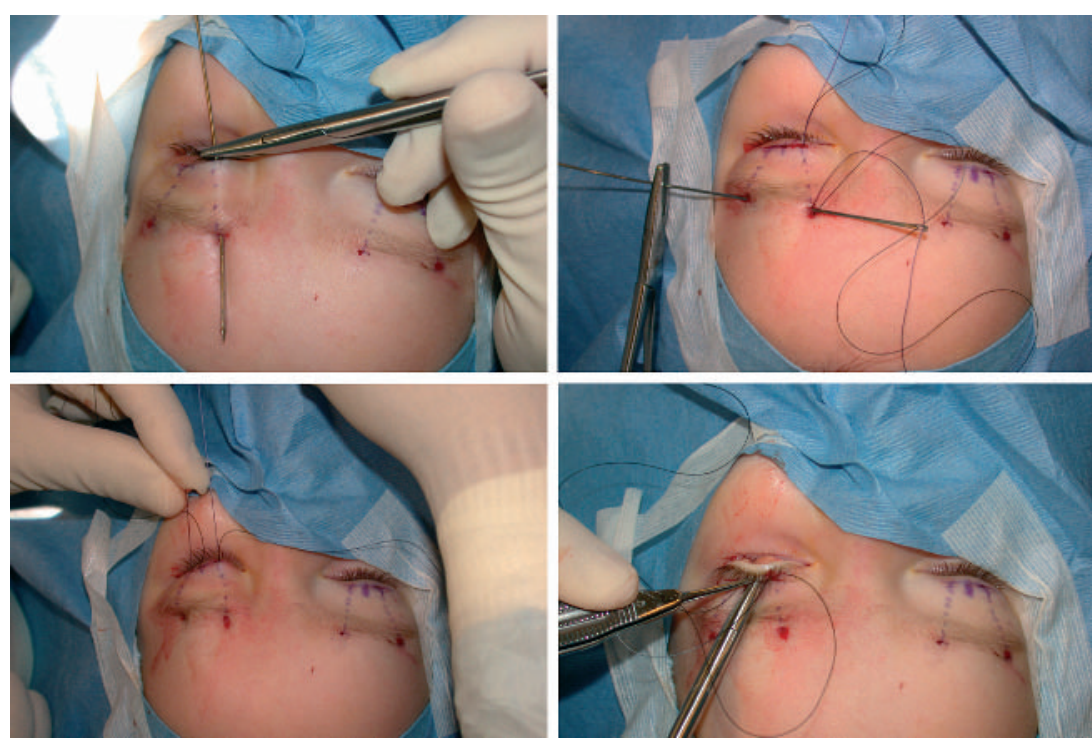

Figure 1 (Top left) A Keith needle, threaded with a Nylon and a Vicryl suture, is passed from one eyelid puncture site to the corresponding eyebrow puncture site in a sub-orbicularis plane. (Top right) The Keith needle, loaded with the sutures, is passed from one eyebrow puncture site to another. (Bottom left) The 4/0 Vicryl suture is manoeuvred in a "sawing" manner with both hands to release the soft tissues at the eyebrow puncture sites to avoid skin dimpling. (Bottom right) The 4/0 Nylon suture is passed from one eyelid puncture site to another taking a partial thickness bite. The eyelid is everted during this tarsal passage to ensure no full thickness penetration.
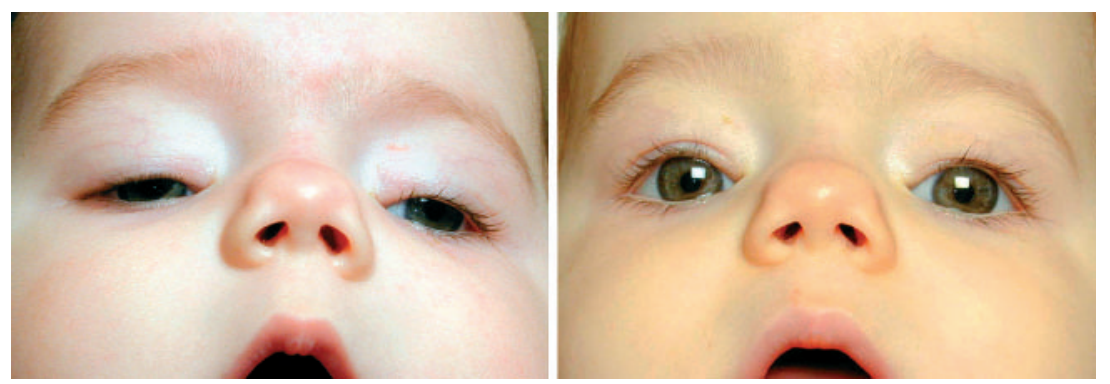

Figure 2 (Left) Preoperative picture of a 1 year old girl with bilateral congenital ptosis and a chinup position. The child has bilateral poor levator function. (Right). Postoperative picture of the patient after bilateral frontalis suspension using the described technique. Both the eyelids are adequately elevated with a satisfactory contour although the chin-up position is not totally ameliorated.
C-C Yip, R A Goldberg, T L Cook, J D McCann

Orbital and Ophthalmic Plastic Surgery Division, Jules Stein Eye Institute, UCLA School of Medicine, Los Angeles, CA, USA

C-C Yip The Eye Institute, National Health Care Group,
Singapore. Tan Tock Seng Hospital

Correspondence to: Robert A Goldberg, MD, FACS, Jules Stein Eye Institute, Orbital and Ophthalmic Plastic Surgery Division, 100 Stein Plaza, PO Box 957006, Los Angeles, CA 90095-7006, USA Goldberg@jsei.ucla.edu

Accepted for publication 26 June 2003

The authors have no financial interest in this paper.

\section{References}

1 Wagner RS, Mauriello JA Jr, Nelson LB, et al. Treatment of congenital ptosis with frontalis suspension: a comparison of suspensory materials. Ophthalmology 1984;91:245-8.

2 Steinkogler FJ, Kuchar A, Huber E, et al. GoreTex soft-tissue patch frontalis suspension technique in congenital ptosis and in blepharophimosis-ptosis syndrome. Plast Reconstr Surg 1993;92:1057-60.

3 Crawford JS. Repair of ptosis using frontalis muscle and fascia lata: a 20-year review. Ophthalmic Surg 1977;8:31-40.

4 Wilson ME, Johnson RW. Congenital ptosis. Long-term results of treatment using lyophilized fascia lata for frontalis suspensions. Ophthalmology 1991;98:1234-7.

\section{Spontaneous resolution of sixth nerve palsy with ipsilateral cavernous carotid dolichoectasia}

A 73 year old man was evaluated for the sudden onset of binocular horizontal diplopia which was worse in left gaze and which began 1 day before initial examination. He also complained of a dull headache over his left brow. He had a medical history of hip and knee surgery and was taking no medications. He was a 50 pack a year smoker but had no other history of vascular disease, including hypertension and diabetes mellitus. He had no previous history of strabismus or eye muscle surgery. His referring ophthalmologist was concerned about giant cell arteritis (GCA) and ordered a Westergren erythrocyte sedimentation rate test, which was $15 \mathrm{~mm}$ in the first hour.

Additional history revealed that he had no jaw claudication, scalp tenderness, or other symptoms of GCA. Visual acuity was 20/25 in both eyes and his colour vision and confrontation visual fields were normal. His pupils were equal in size and briskly reactive without a relative afferent pupillary defect. A left abduction deficit was noted (fig l) and, with alternate cover testing, there was a 10 prism dioptre esotropia in primary position and at near, which increased to 20 prism dioptres on left gaze and decreased to 2 prism dioptres in right gaze. He had slowed saccades of the left lateral rectus muscle. There was no evidence of ptosis or ocular motor synkinesis. The remainder of his cranial nerve and dilated fundus examination were normal. Magnetic resonance imaging (MRI) (fig 2) and magnetic resonance angiography (MRA) (fig 3) of the brain revealed a lateral course of the left cavernous carotid artery consistent with dolichoectasia. 

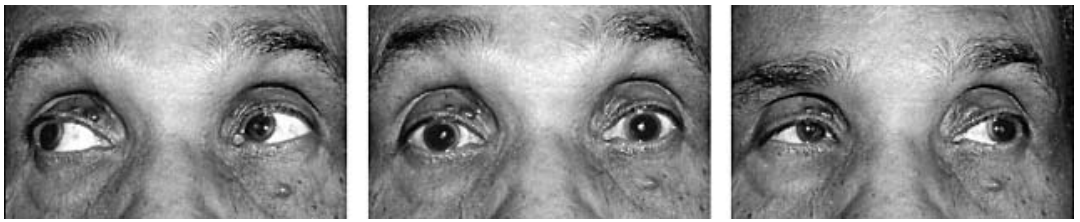

Figure 1 Ocular motility testing reveals a left abduction deficit.

Follow up examination 1 month later revealed no history of variability of the diplopia and no change in the ocular misalignment; however, over the next 2 months the patient reported a gradual improvement in symptoms. He returned 3 months after the initial onset of symptoms and his abduction deficit had resolved. There was no evidence of an ocular misalignment with alternate cover testing. Repeat MRI/MRA showed no change in the calibre or position of the left cavernous carotid artery. He has reported no new symptoms with 1 month of additional follow up.

\section{Comment}

Dolichoectasia, or pathological enlargement, of the intracranial arteries is a finding rarely seen with neuroimaging or arteriography. Arteriosclerosis, with thinning of the media and defects in the internal elastic laminae of the vessel walls, is thought to predispose to progressive enlargement of the vessel lumen. Ectasia of the intracranial arteries is believed to cause symptoms because of compression of adjacent structures and/or ischaemia secondary to intraluminal thrombus formation and blockade of perforating vessels along the length of the dolichoectatic vessel.

Dolichoectasia of the cavernous carotid artery has been suggested as an infrequent cause of sixth nerve paresis. One in 23 patients with carotid ectasia (in a series of approximately 40000 patients undergoing carotid arteriography) was found to have an acute sixth nerve palsy with "good recovery," although the clinical course was not specified. ${ }^{2}$ Ipsilateral dolichoectasia was noted in a 59 year old man with seven episodes of sixth nerve paresis, each lasting between 25 weeks. ${ }^{3}$ The authors did not provide an explanation for the mechanism of recurrence. A single patient with bilateral sixth nerve

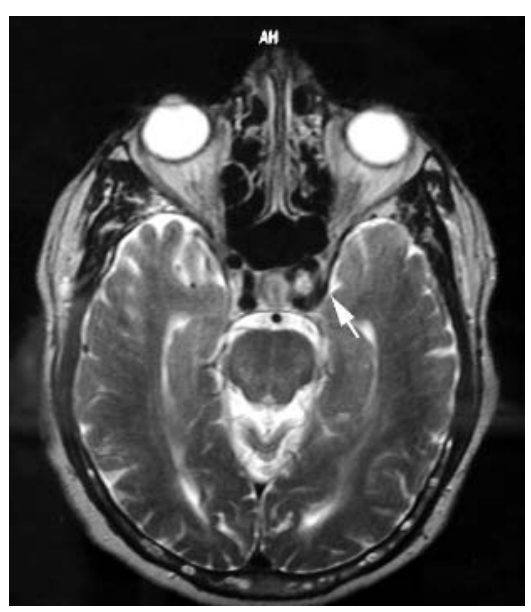

Figure 2 T2 weighted MRI reveals a lateral course of the left cavernous carotid artery (arrow). paresis was reported to have bilateral carotid dolichoectasia as the underlying cause. ${ }^{4}$ However, in the discussion the causal relation of the dolichoectasia, presumably from compression of the carotid artery, was called into question. In addition, dolichoectasia of the cavernous carotid artery has been noted in patients without ocular motor deficits. ${ }^{1}$

This patient's left sixth nerve paresis spontaneously resolved 3 months after the initial onset of symptoms. Despite the presence of ipsilateral cavernous carotid dolichoectasia, his clinical course is most consistent with that of a vasculopathic sixth nerve paresis. Whether the dolichoectasia was causative or an incidental finding is not clear in this patient. Arterial dissection in a previously ectatic vessel has been suggested as an explanation for the acute onset of symptoms in patients with dolichoectasias; however, no evidence of arterial dissection was seen in this patient's MRI/MRA. Ischaemia of the vaso vasorum of the sixth nerve, perhaps because of intraluminal thrombus formation, may have resulted in a vasculopathic sixth nerve palsy, but there was no evidence of thrombus formation on the MRI/MRA.

Because the causative mechanism in patients with persistent sixth nerve paresis from presumed dolichoectasia is not certain treatment guidelines are not clear. Monocular occlusion and prism therapy may provide temporary or long lasting relief of diplopia. Neurosugrical intervention to relieve mechanical compression between the cavernous carotid artery is a difficult, potentially life threatening, procedure. Extraocular muscle surgery may correct the ocular misalignment, without treating the underlying mechanical compression, with uncertain long term benefit. Spontaneous resolution of the left sixth nerve palsy in this patient with ipsilateral carotid dolichoectasia suggests that a period of careful observation should

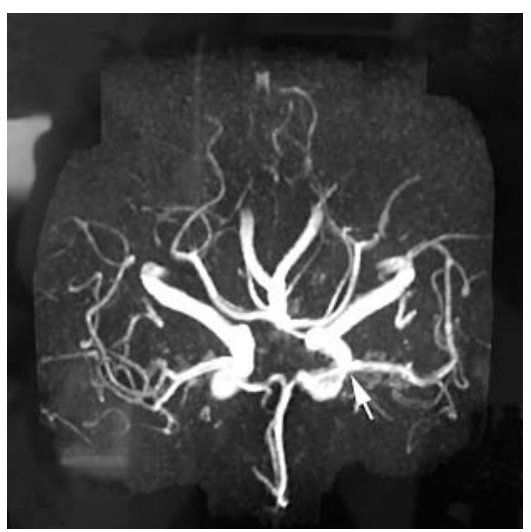

Figure 3 MRA of the circle of Willis shows the lateral course of the left cavernous carotid artery (arrow). precede plans for surgical correction of the ocular misalignment.

R Foroozan

Neuro-Ophthalmology Service, Cullen Eye Institute, Baylor College of Medicine, 6565 Fannin NC-205, Houston, TX 77030, USA; foroozan@bcm.tmc.edu

Accepted for publication 28 June 2003

The author has no proprietary interest in any contents within this manuscript

\section{References}

1 Anson JA, Lawton MT, Spetzler RF. Characteristics and surgical treatment of dolichoectatic and fusiform aneurysms. J Neurosurg 1996;84:185-93.

2 Yu YL, Moseley IF, Pullicino P, et al. The clinical picture of ectasia of the intracerebral arteries. J Neurol Neurosurg Psychiatry 1982;45:29-36.

3 Blumenthal EZ, Gomori JM, Dotan S. Recurrent abducens nerve palsy caused by dolichoectasia of the cavernous internal carotid artery. Am J Ophthalmol 1997; 124:255-7.

4 Neugebauer A, Kirsch A, Fricke J, et al. New onset of crossed eyes in an adult. Surv Ophthalmol 2001;45:335-44.

5 Mizutani T, Aruga T. "Dolichoectatic" intracranial vertebrobasilar dissecting aneurysm. Neurosurgery 1992;31:765-73; discussion 773.

\section{Intravitreal triamcinolone acetonide as treatment for extensive exudative retinal detachment}

Coats' disease or entities like Coats' disease are characterised by a marked exudative retinal detachment with leakage of peripheral retinal vessels, pronounced subretinal deposition of lipids, and eventual progression to total retinal detachment. In some situations, iris neovascularisation can occur, suggesting an angiogenetic component in the course of the disease. In view of the subretinal exudation from the leaking retinal vessels and the possibly neovascular aspect in the disease process, the purpose of this study was to evaluate whether intravitreal triamcinolone acetonide may be helpful in the treatment of Coats' like diseases. Intravitreal triamcinolone acetonide has recently been shown to have a pronounced anti-oedematous and possibly anti-angiogenic effect in diseases such as diffuse diabetic macular oedema, proliferative diabetic retinopathy, chronic pre-phthisical ocular hypotony, chronic uveitis, and persistent pseudophakic cystoid macular oedema.

\section{Case report}

The prospective clinical interventional case report included two patients who presented with subtotal exudative retinal detachment. A 39 year old female patient showed an extensive exudative retinal detachment extending from the temporal periphery of the fundus to the macular region. Diagnosed with Coats' disease in her early teens, she had received multiple xenon arc coagulations as well as argon laser coagulations. Her visual acuity was 0.02 . Intraocular pressure measured $13 \mathrm{~mm} \mathrm{Hg}$. The second patient was a 75 year old woman presenting with almost total exudative retinal detachment with marked subretinal deposition of lipids. Visual acuity was 0.05 . Intraocular pressure measured $21 \mathrm{~mm} \mathrm{Hg}$. 
Under topical anaesthesia, both patients received an intravitreal injection of $25 \mathrm{mg}$ triamcinolone acetonide, which was transconjunctivally applied through the pars plana. Both patients were fully informed about the experimental character of the treatment and had given informed consent. The technique has already been described in detail. $^{2}$ Follow up after the injections were 2 years and 10 months, respectively.

After the injection, visual acuity remained unchanged, and intraocular pressure ranged between 10 and $15 \mathrm{~mm} \mathrm{Hg}$ in the first patient. In the second patient, visual acuity eventually decreased to light perception after the injection. Intraocular pressure ranged between 19 and $25 \mathrm{~mm} \mathrm{Hg}$. In both patients, flare in the anterior chamber and in the vitreous cavity, as assessed by slit lamp biomicroscopy, decreased markedly. Upon ophthalmoscopy, the extent of exudative retinal detachment increased slightly, with subretinal strands being stronger and more visible.

\section{Comment}

Although intravitreal triamcinolone acetonide can markedly reduce retinal oedema in eyes with diffuse diabetic macular oedema and pseudophakic cystoid macular oedema, intravitreal triamcinolone acetonide was not pronouncedly helpful in reducing subretinal oedema and re-attaching the retina in the two patients presented in this study. This result was unexpected in view of the presumed anti-phlogistic and anti-proliferative effect of steroids such as triamcinolone acetonide. $^{12}$ It may be explained by a previous experimental study in which triamcinolone acetonide inhibited the proliferation of rabbit dermal and conjunctival fibroblasts in cell culture at $150 \mathrm{mg} / \mathrm{l}$, but paradoxically increased proliferation almost twofold at concentrations ranging from $1-30 \mathrm{mg} / \mathrm{l}$ under identical culture conditions. ${ }^{3}$ As long as the influence of steroids on the proliferation of retinal pigment epithelium cells is unclear, intravitreal triamcinolone acetonide may thus cautiously be taken as adjunct treatment of marked exudative retinal detachment in eyes with a Coats' like disease. A similar conclusion was drawn in a recent study on eyes with proliferative vitreoretinopathy, in which pars plana vitrectomy was combined with an intravitreal injection of $25 \mathrm{mg}$ of triamcinolone acetonide, and in which unexpectedly, the recurrence rate of proliferative vitreoretinopathy was not markedly diminished. ${ }^{4}$ Future randomised studies as well as investigations evaluating the effect of intravitreal steroids combined with other drugs such as 5-fluorouracil on the proliferation of retinal pigment epithelium cells and retinal detachment rate $e^{5}$ may be warranted.

J B Jonas Department of Ophthalmology, Faculty of Clinical Medicine Mannheim, Ruprecht-Karls-University of Heidelberg, Germany

Correspondence to: Dr J Jonas, UniversitätsAugenklinik, Theodor-Kutzer-Ufer 1-3, 68167 Mannheim, Germany: Jost.Jonas@augen.ma.
uni-heidelberg.de

Accepted for publication 1 July 2003

\section{References}

1 Machemer R, Sugita G, Tano Y. Treatment of intraocular proliferations with intravitreal steroids. Trans Am Ophthalmol Soc 1979;77:171-80.
2 Jonas JB, Söfker A. Intraocular injection of crystalline cortisone as adjunctive treatment of diabetic macular edema. Am J Ophthalmol 2001;132:425-7.

3 Blumenkranz MS, Claflin A, Hajek AS. Selection of therapeutic agents for intraocular proliferative disease. Cell culture evaluation. Arch Ophthalmol 1984; 102:598-604.

4 Jonas JB, Söfker A, Hayler J, et al. Intravitreal crystalline triamcinolone acetonide as additional tool in pars plana vitrectomy for complicated proliferative vitreoretinopathy? Acta Ophthalmol 2003; (in press).

5 Berger AS, Cheng CK, Pearson PA, et al. Intravitreal sustained release corticosteroid-5fluoruracil conjugate in the treatment of experimental proliferate vitreoretinopathy. Invest Ophthalmol Vis Sci 1996;37:2318-25.

\section{Long term efficacy and safety of botulinum toxin A injection for crocodile tears syndrome}

Gustatory lacrimation, also called crocodile tears syndrome (CTS), is an autonomic synkinesia in which patients tear excessively in response to salivary stimuli. It occurs most commonly in the setting of idiopathic or traumatic facial palsy and is thought to result from aberrant reinnervation of the lacrimal gland by salivary efferent fibres from either the seventh or ninth cranial nerve. Many patients tolerate CTS and require no intervention. For patients who cannot tolerate CTS, past treatments have included anticholinergic drugs, subtotal resection of the palpebral lobe of the lacrimal gland, and resection of the tympanic nerve proximal to the lesser superficial petrosal nerve. None of these approaches is optimal because of limited efficacy, morbidity, or both.

Injection of botulinum toxin A has been shown to be effective for a host of disorders characterised by involuntary muscle spasms, including blepharospasm, hemifacial spasm, and torticollis. Botulinum toxin A also has been used to treat a number of localised autonomic disorders, including axillary hyperhidrosis, palmar hyperhidrosis, and Frey syndrome. ${ }^{1}$ In 1998, Boroojerdi et al reported the successful treatment of CTS by injection of botulinum toxin A directly into the lacrimal gland. ${ }^{2}$ Since then, there have been five reports of similar treatments, all of which were successful. ${ }^{3-7}$ All of these studies report complete or near complete resolution of the syndrome within a week with only infrequent, minor, and reversible complications. We now report a patient with CTS who has been successfully managed for 3 years with injections of botulinum toxin A.

\section{Case report}

A 38 year old man presented in July of 2000 with a 6 month history of right sided tearing and hyperhidrosis of the auriculotemporal region when eating or when hungry. Fourteen months previously, he had undergone a total right parotidectomy for a mixed tumour of the parotid gland. Immediately after surgery, he had a complete right sided facial palsy and numbness of the right lower face. The facial palsy resolved completely a month later, but the facial numbness persisted. Eight months later, the patient began to experience increased tearing on the right after eating, most notable after eating mints. On examination, the patient had normal facial movement but decreased sensation to light touch in the region of the second division of the trigeminal nerve and spasm of the right lower lid on palpation. In addition, he perspired from the right side of the face and had tearing from the right eye upon eating. His ocular and neurologic examinations were otherwise unremarkable.

In light of the bothersome nature of CTS to this patient, we felt a trial of botulinum toxin A was warranted. Accordingly, after obtaining consent, we injected botulinum toxin A (Botox $2.5 \mathrm{U}$ ) transconjunctivally into the palpebral lobe of the right lacrimal gland under direct visualisation at the slit lamp biomicroscope without complication. The patient's excess epiphora completely resolved within 5 days, and he remained asymptomatic for 11 months. He has subsequently required injections of botulinum toxin A every 7-11 months, experiencing relief of symptoms with each injection. There have been no complications from any of the injections.

\section{Comment}

Several different groups have now reported a total of 12 cases of CTS treated with botulinum toxin $\mathrm{A}^{2-7}$ All of the patients reported have had complete or near complete short term resolution of symptoms with doses of botulinum toxin A (Botox) ranging from 2.5-60 U. The higher doses seem to have no additional benefit in terms of efficacy or duration.

Injection of botulinum toxin A for CTS appears to be safe, although minor complications occasionally occur. Two of the patients injected transcutaneously developed ptosis, one accompanied by a superior rectus palsy, ${ }^{5} 7$ whereas two others developed dryness of the injected eye. ${ }^{7}$ These complications resolved over several months. No cases of ptosis or extraocular muscle weakness have been reported after transconjunctival injection, and our patient has had no complications with any of his injections over the last 3 years.

As with all new treatments, there are concerns about long term efficacy and safety. Botox has been found both safe and effective at the neuromuscular junction, but its long term effects on the peripheral autonomic system are unknown and one might postulate that the repeated minor trauma of the injection could eventually impair lacrimal gland function. In light of these concerns, it is encouraging to be able to report that repeated injections of botulinum toxin A continue to be effective in controlling this patient's CTS for 3 years without complication.

D E Barañano

Departments of Neuroscience and Medicine, Johns Hopkins School of Medicine, Baltimore, MD, USA

N R Miller Wilmer Eye Institute, Division of Neuroophthalmology, Departments of Ophthalmology and Neurology, Johns Hopkins School of Medicine, Baltimore, MD USA

Correspondence to: Dr Neil R. Miller, MD, Maumenee B-109, Johns Hopkins Hospital, 600 North Wolfe Street, Baltimore, 21287, USA; nrmiller@jhmi.edu

Accepted for publication 7 July 2003

\section{References}

1 Naumann M. Evidence-based medicine: botulinum toxin in focal hyperhidrosis. J Neurol $2001 ; 248: 31-3$. 
2 Boroojerdi B, Ferbert A, Schwarz M, et al Botulinum toxin treatment of synkinesia and hyperlacrimation after facial palsy. J Neurol Neurosurg Psychiatry 1998:65:111-4.

3 Riemann R, Pfennigsdorf S, Riemann E, et al Successful treatment of crocodile tears by injection of botulinum toxin into the lacrimal gland: a case report. Ophthalmology 1999; 106:2322-4.

4 Hofmann RJ. Treatment of Frey's syndrome (gustatory sweating) and 'crocodile tears' (gustatory epiphora) with purified botulinum toxin. Ophthal Plast Reconstr Surg 2000;16:289-91.

5 Keegan DJ, Geerling G, Lee JP, et al. Botulinum toxin treatment for hyperlacrimation secondary to aberrant regenerated seventh nerve palsy or salivary gland transplantation. $\mathrm{Br} J$ Ophthalmol 2002;86:43-6.

6 Yavuzer R, Basterzi Y, Akata F. Botulinum toxin A for the treatment of crocodile tears. Plast Reconstr Surg 2002;1 10:369-70.

7 Montoya FJ, Riddell CE, Caesar R, et al. Treatment of gustatory hyperlacrimation (crocodile tears) with injection of botulinum toxin into the lacrimal gland. Eye 2002;16:705-9.

\section{Retinal arterial collapse pressure in eyes with retinal arterial occlusive diseases}

Retinal arterial occlusions may be primarily or secondarily associated with low retinal arterial pressure. Based on previous ophthalmodynamometric studies ${ }^{1-6}$ the purpose of the present study is to estimate the retinal vessel pressure in patients with central retinal artery or branch retinal artery occlusions and patients with amaurosis fugax.

\section{Case report}

This prospective clinical non-interventional comparative study included nine eyes of seven patients (mean age 68.8 (SD 13.7) years) with central retinal artery occlusion $(\mathrm{n}=1$ eye), branch retinal artery occlusion $(\mathrm{n}=2)$, ischaemic ophthalmopathy $(\mathrm{n}=2)$, or amaurosis fugax $(n=4)$. An age matched control group consisted of 27 eyes of 21 subjects attending the hospital because of cataract or refractive problems. After medical pupil dilatation, a conventional Goldmann contact lens fitted with a pressure sensor

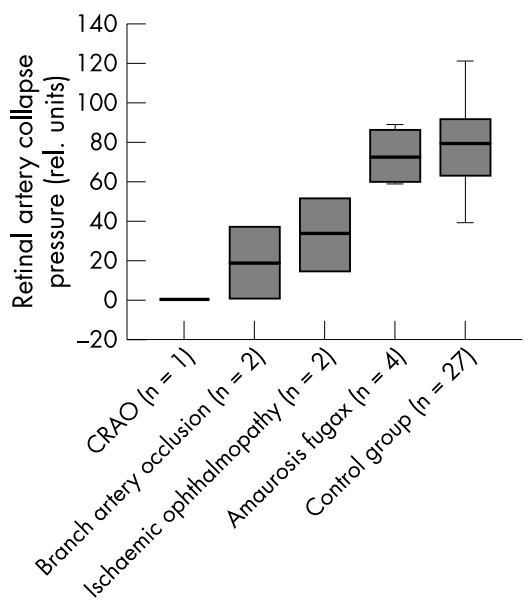

Figure 1 Box plots showing the retinal artery collapse pressure in the study group of patients with central retinal artery occlusion (CRAO), branch retinal artery occlusion, ischaemic ophthalmopathy, amaurosis fugax, and in the control group. mounted into the holding ring was put onto the cornea. By slightly pressing the contact lens, pressure was applied onto the globe, and the pressure when the central retinal vein or artery started to pulsate was noted. The methods applied in the study adhered to the tenets of the declaration of Helsinki. The method has already been described in detail. ${ }^{\circ}$

In the study group, central retinal artery collapse pressure measured 43.9 (SD 33.4) arbitrary units (AU) and was significantly $(p=0.004)$ lower than in the control group (78.0 (SD 19.2) AU) (fig 1). Within the study group, central retinal artery collapse pressure was lowest in the eye with central retinal artery occlusion, showing a pulse synchronic movement of the erythrocyte column in the vessel without applying any pressure onto the globe. In the two eyes with branch retinal artery occlusion, collapse pressure in the arterial branch lying in the oedematous part of the fundus measured $36.7 \mathrm{AU}$ and $0 \mathrm{AU}$ respectively. These values were significantly $(\mathrm{p}=0.005)$ lower than the values obtained in the control group (fig 1). In the eyes with branch retinal artery occlusion, collapse pressure in the arterial branch in the nonoedematous part of the fundus was in the normal range (93.1 AU and 93.3 AU, respectively). In the patient suffering from ischaemic ophthalmopathy, central retinal artery collapse pressure was lower in the eye more severely affected than in the contralateral eye (14.7 AU v 51.7 AU). Both values were significantly $(\mathrm{p}=0.02)$ lower than the values of the control group. In the eyes with amaurosis fugax, mean central retinal artery collapse pressure measured 73.0 (SD 15.4) AU which was not significantly $(p=0.55)$ different from central retinal artery collapse pressure in the control group (fig 1). Central retinal vein collapse pressure did not vary significantly between the study groups and the control group (8.8 (SD 12.2) AU v 6.1 (SD 8.4) $\mathrm{AU} ; \mathrm{p}=0.54$ )

\section{Comment}

Central retinal artery collapse pressure as determined by the new ophthalmodynamometric technique was significantly lower in eyes with retinal artery occlusive diseases than in normal eyes (fig 1). Correspondingly, in the eyes with branch retinal artery occlusions, measurements were lower in the arterial branch affected by the occlusion than in the retinal artery branch with intact perfusion. As a corollary, in the patient suffering from ischaemic ophthalmopathy, the central retinal artery collapse pressure was lower in the eye more severely affected because of a complete stenosis of the carotid artery than in the contralateral eye. Interestingly, the eyes with amaurosis fugax did not show significantly lower measurements than normal eyes. This agrees with previous studies using other ophthalmodynamometric techniques for evaluation of carotid artery perfusion. ${ }^{4}$ In conclusion, using a new ophthalmodynamometer with biomicroscopic observation of central retinal vessels during the examination, central retinal artery collapse pressure measurements were significantly lower in eyes with retinal arterial occlusive diseases than in normal eyes. Future studies may show whether determination of the central retinal artery collapse pressure in patients with increased risk for retinal arterial occlusions may be suitable to predict which patients have a higher risk for eventual retinal artery occlusion compared with other patients with a similar risk profile.

J B Jonas

Correspondence to: J B Jonas, Department of Ophthalmology, Faculty of Clinical Medicine Mannheim, University of Heidelberg, Theodor-KutzerUfer 1-3, 68167 Mannheim, Germany; Jost.Jonas@augen.ma.uni-heidelberg.de

Accepted for publication 7 July 2003

Proprietary interest: none.

\section{References}

Rios-Montenegro EN, Anderson DR, David NJ. Intracranial pressure and ocular hemodynamics. Arch Ophthalmol 1973;89:52-8.

2 Yablonski M. A new fundus lens ophthalmodynamometer. Am J Ophthalmo 1978:86:644-7

3 Krieglstein GK, da Silva FA. Comparative measurements of the ophthalmic arterial pressure using the Mikuni dynamometer and the Stepanikarteriotonograph. Albrecht Von Graefes Arch Klin Exp Ophthalmol 1979;212:77-91.

4 Zaret CR, Sacks JG, Holm PW. Suction ophthalmodynamometry in the diagnosis of carotid stenosis. Ophthalmology 1979;86:1510-12.

5 Entenmann B, Robert YC, Pirani P, et al. Contact lens tonometry-application in humans. Invest Ophthalmol Vis Sci 1997;38:2447-51.

6 Jonas JB. Reproducibility of ophthalmodynamometric measurements of the central retinal artery and vein collapse pressure. Br J Ophthalmol 2003;87:577-9.

\section{Modified self sealing sclerotomy for drainage of subretinal fluid during scleral buckling surgery}

Drainage of subretinal fluid is probably the most dangerous step in scleral buckling surgery for uncomplicated retinal detachment. The most common complications include subretinal haemorrhage, retinal perforation, and vitreoretinal incarceration. ${ }^{12}$ Sclerotomy to drain subretinal fluid is traditionally made with a sharp blade, diathermy to the sclera and choroid is performed, followed by perforation of the choroid to allow drainage of subretinal fluid. Suture of the sclerotomy at the end of the procedure has been recommended to avoid retinal incarceration.

The purpose of this study was to determine the effectiveness and safety of a modified self sealing sclerotomy technique for drainage of subretinal fluid during scleral buckling surgery

\section{Patients and methods}

Twenty consecutive patients undergoing scleral buckling for primary rhegmatogenous retinal detachment from two vitreoretinal surgery centres were enrolled in this prospective study. A scleral buckling procedure was performed using a circumferential scleral band (Mira 240, Mira, Waltham, MA, USA) sutured with the posterior border located $12 \mathrm{~mm}$ posterior to the limbus, and adding any necessary segmental sponges (Mira). Cryoretinopexy was performed using a CTU Ophthalmic Cryo Unit (Keeler, London, UK) to seal retinal tears. After surgery, sulfur hexafluoride $\left(\mathrm{SF}_{6}\right)$ gas was used in all patients. The drainage site was chosen based on retinal elevation, as shown by intraoperatative retinal examination with indirect 

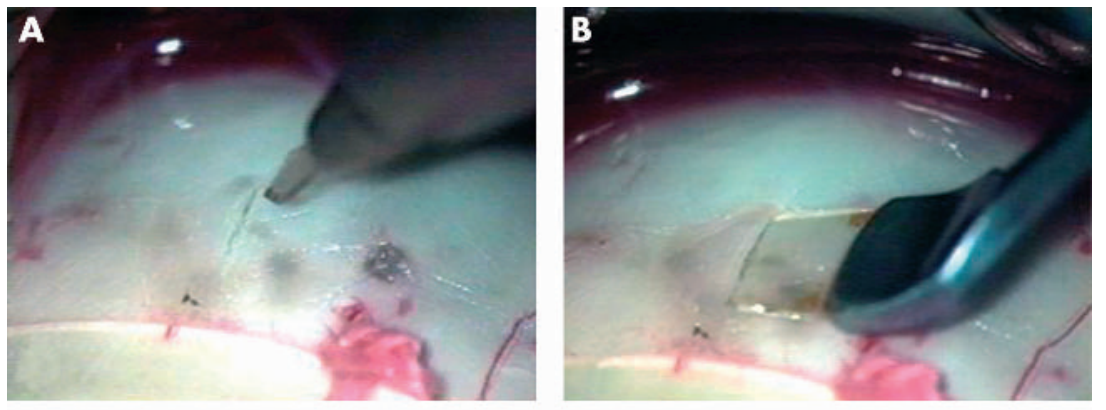

2 Wilkinson $\mathrm{CP}$, Bradford RH Jr. Complications of draining subretinal fluid. Retina 1984;4:1-4.

3 Fine IH, Fichman RA, Grabow HB. Clear Cornea Cataract Surgery and Topical Anesthesia. Thorofare, NJ: Slack Inc, 1993.

4 Chen JC. Sutureless pars plana vitrectomy through self-sealing sclerotomies. Arch Ophthalmol $1996 ; 114: 1273-5$.

5 Kwok AK, Tham CC, Lam DS, Li M, et al. Modified sutureless sclerotomies in pars plana vitrectomy. Am J Ophthalmol $1999 ; 127: 731-3$.
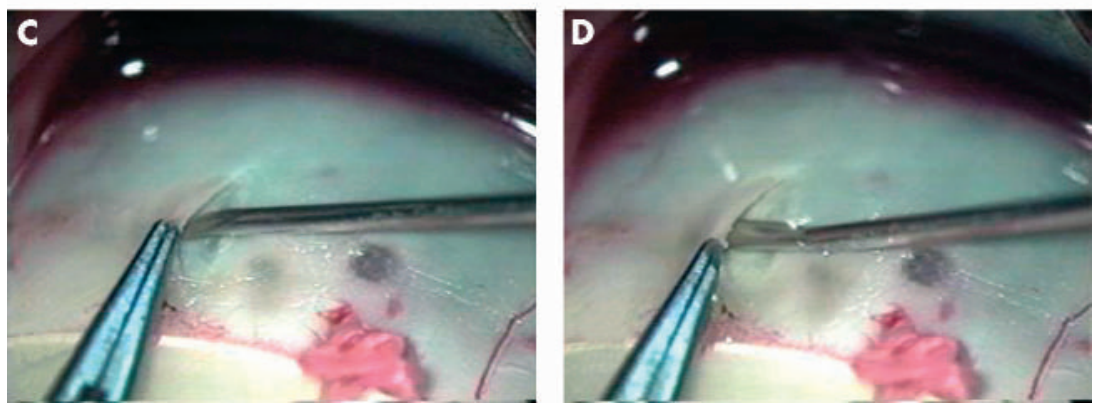

Figure 1 (A) A 3-4 mm half depth scleral incision is created perpendicular to limbus using an angled bevel up blade with its sharp advancing edge directed perpendicular to the sclera surface. (B) With a crescent knife, a $3 \mathrm{~mm}$ tunnel incision is then made to create a scleral flap parallel to the limbus. (C) The scleral flap is retracted, and a 27 gauge needle is then used to perforate the scleral bed and choroid. (D) Subretinal fluid is expressed and dried with a cotton swab.

ophthalmoscopy. A $3-4 \mathrm{~mm}$ half depth scleral incision was created perpendicular to the limbus using an angled bevel up blade (Alcon Laboratories, Fort Worth, TX, USA) with its sharp advancing edge directed perperdicular to the scleral surface (fig lA). With a crescent knife, a $3 \mathrm{~mm}$ tunnel incision was then made to create a scleral flap parallel to the limbus (fig 1B). The scleral flap was retracted, and a 27 gauge needle was used to perforate the scleral bed and choroid (fig IC). Subretinal fluid was expressed (fig ID) and dried with a cotton swab. In all cases, the surgical wound was inspected for adequate closure at the end of the operation. Drainage of subretinal fluid and complications associated with this technique were assessed by intraoperative binocular indirect ophthalmoscopy, and recorded on surgical reports and records from postoperative visits.

\section{Results}

All 20 patients had self sealing sclerotomy that did not require suturing at the end of surgery. Treating the choroid with diathermy or cautery was not necesary in any case, and the planned needle drainage procedure was successful in all patients. Drainage was slow and gradual. No serious complications were linked to the drainage of subretinal fluid with this technique. Spontaneous drainage was seen in one patient with thin sclera, and two limited subretinal haemorrhage that did not migrate to the posterior pole were observed.

\section{Comments}

We describe the results and complications of 20 scleral buckling procedures for primary rhegmatogenous retinal detachments in which subretinal fluid was drained using a sutureless sclerotomy technique. A self sealing incision with a stepped wound construction is not new to ophthalmology. Cataract and vitreoretinal surgeons pioneered this technique as part of phacoemulsification cataract extraction and vitreous surgeries..$^{3-5}$ Possible advantages to this type of incision include shortened operating time and reduced incidence of postoperative wound leak.

This new way of constructing the sclerotomy for drainage has many advantages, and we propose it as an alternative to standard sclerotomy incision to drain subretinal fluid during scleral buckling surgery for uncomplicated retinal detachments.

J B Yepez, J Cedeño de Yepez, A Valero The Retina and Vitreous Service, Clinica de Ojos de Maracaibo, Maracaibo, Venezuela

J F Arevalo

Retina and Vitreous Service, Clinica Oftalmologica Centro Caracas, Caracas, Venezuela

Correspondence to: Dr J Fernando Arevalo, Clinica Oftalmologica Centro Caracas, Centro Caracas PH-1, Av Panteon, San Bernardino, Caracas 1010, Venezuela; areval1@telcel.net.ve

Presented in part at the Vitreous Society Annual Meeting, Fajardo, Puerto Rico, November 2001.

\section{doi: 10.1136/bjo.2003.029272}

Accepted for publication 9 July 2003

Supported in part by the Fundacion Arevalo-Coutinho para la Investigacion en Oftalmologia (FACO), Caracas, Venezuela.

The authors have no proprietary or financial interest in any products or techniques described in this article.

\section{References}

1 Hilton GF, Grizzard WS, Avins LR, et al. The drainage of subretinal fluid: a randomized controlled clinical trial. Retina 1981;1:271-80.

\section{Conjunctival dendrite in a case of primary herpes simplex infection}

Ocular involvement in primary herpes simplex infection is usually in the form of follicular conjunctivitis, blepharitis, and sometimes corneal involvement in the form of superficial punctuate keratitis, dendrite, or (rarely) geographical ulcer. ${ }^{1}$

We report a case of dendritiform lesion in the conjunctiva in a young girl with primary herpes simplex infection. To the best of our knowledge, conjunctival dendritiform lesion has not been reported before in primary herpes simplex infection.

\section{Case report}

A 20 year old girl presented to our outpatient department with complaints of redness and discomfort in her right eye of two days' duration. She gave a history of fever of one week's duration followed by appearance of vesicles at the right side angle of the mouth and on the right upper lid. Past ocular and systemic history was unremarkable.

Visual acuity was 6/6 unaided in both the eyes. There were vesicles at the angle of the mouth (fig lA) and on the right upper lid. Slit lamp examination of the right eye with fluorescein staining revealed a dendritiform pattern of staining in the lower bulbar conjunctiva (fig 1). Cornea was clear and rest of the anterior segment was unremarkable. Left eye examination was unremarkable. Fundus examination in both the eyes was within normal limits. The patient was advised to use topical acyclovir 3\% eye ointment five times a day and tablet acyclovir $400 \mathrm{mg}$ five times a day.

On follow up after two days, there was superficial punctuate keratitis in the inferior half of the cornea in the right eye. The patient was asked to continue the same medication. One week later, the vesicles were absent and the conjunctiva and cornea were clear. The medication was discontinued.

Darouger et al, in a study of primary herpes simplex ocular infection, found $64 \%$ of the patients to be over fifteen years of age. ${ }^{2}$ Follicular conjunctivitis (7\%), blepharoconjunctivitis (16\%), and corneal dendritic ulcers (15\%) were some of the lesions reported. Appearance of a dendritic ulcer on the conjunctiva, to the best of our knowledge, has not been reported in primary herpes simplex infection.

Dendritic lesions on histopathological study show that they are composed of round epithelial cells and variable sized syncytia containing bizarre shaped nuclei. The epithelial cells contain viral DNA. ${ }^{3}$ Recurrent infection with the virus in the form of epithelial keratitis commonly produces dendritic lesions on the cornea. 


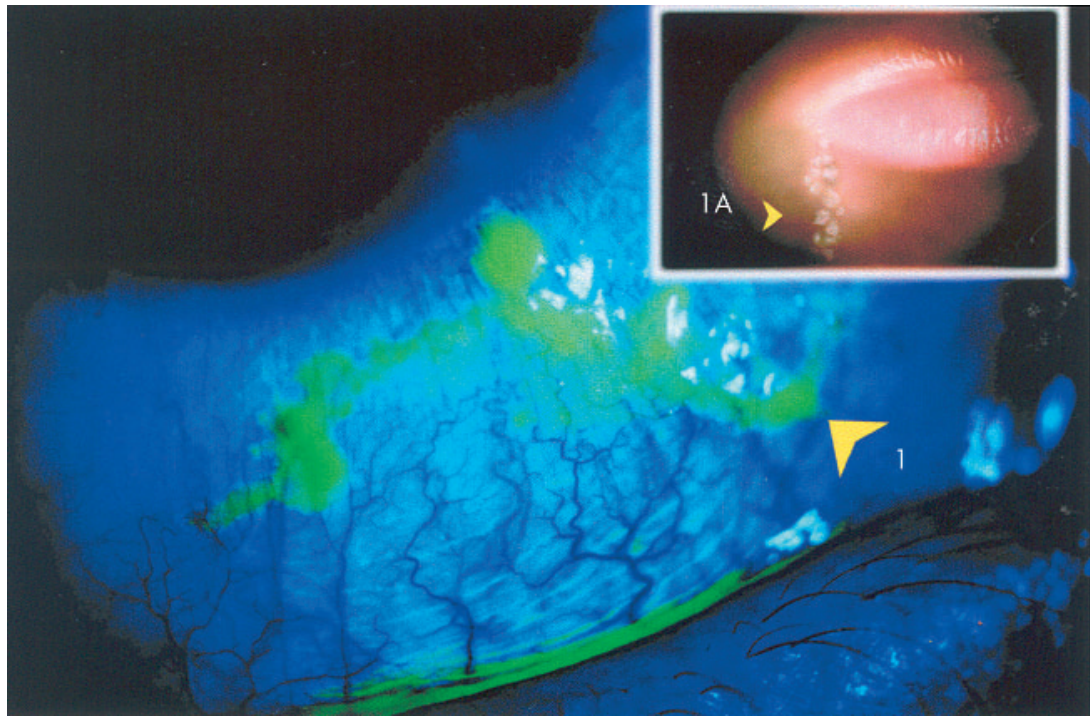

Figure 1 Dendritiform pattern of staining in the lower bulbar conjunctiva. (A) Vesicles at the angle of the mouth.

Conjunctival dendrite is an interesting and apparently rarely reported lesion.

U Sridhar

Cornea Services, ICARE Eye Hospital, Noida, UP, India

Y Bansal

Dept of Optometry, ICARE Eye Hospital, Noida, UP, India

S Choudhury

Training Centre, ICARE Eye Hospital, Noida, UP, India

A K Gupta Academic Director ICARE Eye Hospital, Noida, UP, India

Correspondence to: Dr U Sridhar, ICARE Eye Hospital E-3A Sector 26, Noida, UP, India; u_sridhar@ yahoo.com

Accepted for publication 7 July 2003 The authors have no proprietory interests in the products mentioned in the article.

\section{References}

1 Leisegang TJ, Melton J J III, Daly PJ, et al. Epidemiology of ocular herpes simplex. Arch Ophthalmol 1989:107:1155-9.

2 Darouger S, Wishart MS, Viswalingam ND. Epidemiological and clinical features of primary herpes simplex ocular infection. Br J Ophthalmol 1985;69:2-6.

3 Tabery HM. Early epithelial changes in recurrent herpes simplex viral keratitis. Acta Ophthalmol Scand 1998;76:349-52.

\section{Severe ocular trauma caused by an ostrich}

The ostrich is a strange and harmless looking bird; however, in Africa attacks by ostriches on humans are not uncommon and sometimes result in death. We recently treated such a patient with an eye injury.

\section{Case report}

A 35 year old male patient presented with an injury sustained from being kicked in the face by an ostrich (Struthio camelus).
On examination the right eye was found to be normal but he had vision of bare light perception on the left with proptosis of the globe and severe chemosis of the conjunctiva. Both upper and lower lids were avulsed medially. There was limitation in all positions of gaze which was more noticeable on attempted abduction (fig 1). The eye was

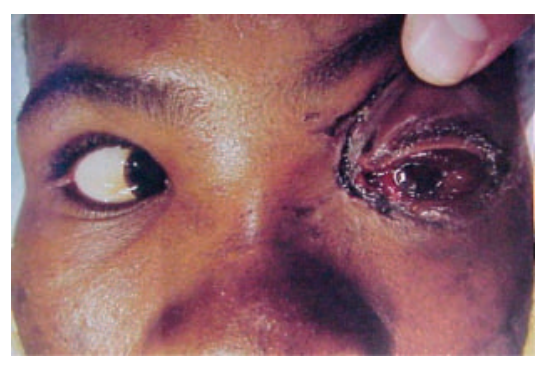

Figure 1 Blunt trauma to left eye with absence of abduction.

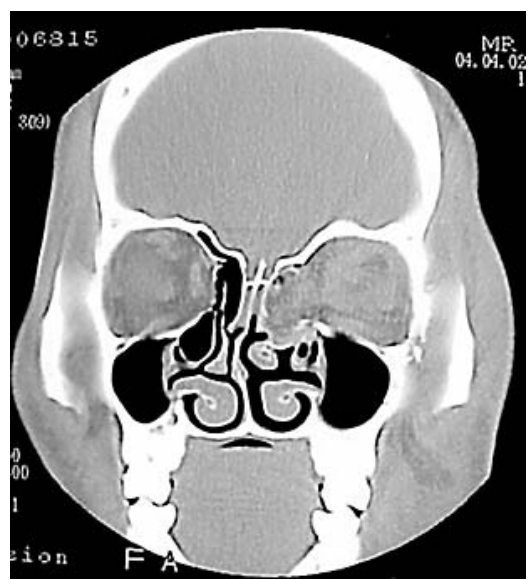

Figure 2 Computed tomography showing blowout fracture into the ethmoid sinus and comminuted lateral wall fracture. soft and he had an oedematous cornea and a full hyphema. The posterior segment could not be visualised.

Computed tomography showed a blowout fracture of the left orbit (fig 2). There was a fracture of the left nasal bone with a comminuted lateral wall fracture. The medial orbital wall was also fractured with opacification of the left ethmoid sinus and herniation of the medial rectus into the sinus.

An intraocular haemorrhage as well as haemorrhage in the retrobulbar space was noted.

Under general anaesthesia, both the upper and lower lids were repaired and the hyphema was washed out. A posterior rupture ${ }^{1}$ was suspected clinically but the site of rupture could not be identified. The eye was subsequently eviscerated.

\section{Comment}

Ostriches usually inflict injury in one of two ways: the more serious injury is that of a slash or laceration, usually to the lower abdomen or limbs, caused by the ostrich kicking in a forward and downward motion with its powerful foot. The toenail of the ostrich is sharp and is used by the ostrich for protection against predators. ${ }^{2}$ The second type of injury is seen more commonly. This occurs when the ostrich uses its bony breast plate as a ram to knock the person to the ground. The ostrich then jumps upon the victim and, because an ostrich weighs 75 $150 \mathrm{~kg}$, this may cause contusion of the torso with rib fractures.

Our patient was bending while repairing a fence when he was kicked by an ostrich. He was struck in the face and sustained extensive facial trauma extending from his nasal bones to his orbital walls and ethmoidal sinus. The trauma also resulted in irreparable blunt trauma to the eye.

The injury caused was severe with no possibility of repair of the globe, and is the only documented case of an eye being lost due to injury by an ostrich

L M Levitz, T R Carmichael, M Nissenbaum University of the Witwatersrand, Department of Ophthalmology, Johannesburg, South Africa

Correspondence to: Professor T R Carmichael, PO Box 752062, Garden View, 2047, South Africa; carmichaeltr@medicine.wits.ac.za

Accepted for publication 7 July 2003

\section{References}

1 Kuhn F, Morris R, Witherspoon CD, et al. A standardized classification of ocular trauma. Ophthalmology 1996; 103:240-3.

2 Salvat SA. Hunters and Hunted of the Savannah. London: Orbis, 1971:155.

\section{Swollen optic discs in a patient with the chromosome 22q11.2 deletion syndrome}

The chromosome 22q11.2 deletion syndrome (22q11DS) encompasses velocardiofacial syndrome (VCFS), DiGeorge syndrome (DGS), and conotruncal anomaly face syndrome (CTFS) and is the result of a microdeletion of chromosome band 22q11.2. It is a relatively common genetic anomaly estimated to occur in approximately one in 4000 live births. The 22q11.2 deletion can arise de novo or can have an autosomal dominant 
inheritance. The condition is thought to be due in part to abnormal development of the pharyngeal arch structures. Clinical findings are extensive and highly variable between patients. Prominent features include cardiac defects, cleft palate, dysmorphic facies, maldescent of the thymus, hypoparathyroidism, immune deficiency and developmental delay. Ocular findings include hypertelorism, ${ }^{1}$ retinal vascular tortuousity, narrow palpebral fissures, small optic nerves, iris nodules, cataracts, ${ }^{2}$ and iris coloboma. ${ }^{3}$ We present a case of a boy who was found to have bilateral disc swelling that led to a diagnosis of 22q11DS.

\section{Case report}

A 14 year old boy presented to the accident and emergency department after having a generalised seizure. He had been admitted to another hospital, 2 days before this, with a sudden onset of collapse and subsequent respiratory arrest. At that time he was noted to have swollen optic discs and a head computed tomography scan done there was reported as normal. He had further seizures after admission to our hospital. Blood testing revealed low plasma calcium and high plasma phosphate levels. The patient had been complaining of back pain in recent months and his mother said that he had shrunk by a couple of centimetres over the past year. She also said that he had always been clumsy and he had been diagnosed as dyslexic at the age of 7. He had a history of behavioural problems which his family said often settled during holidays in the sun. A thoracic spine $x$ ray revealed wedge-shaped fractures of three vertebral bodies. Further blood testing showed a relatively low parathormone level confirming the diagnosis of hypoparathyroidism. He was then referred

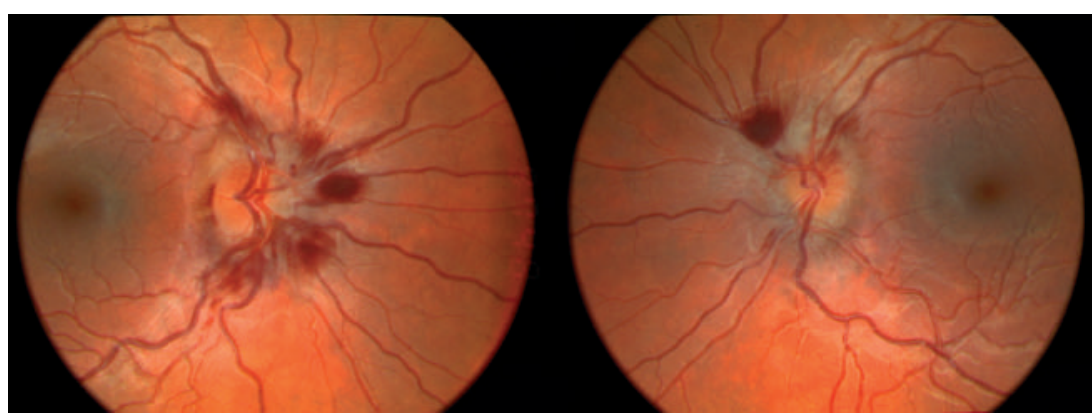

Figure 1 Shows bilateral disc swelling with extensive peripapillary haemorrhages in both eyes.

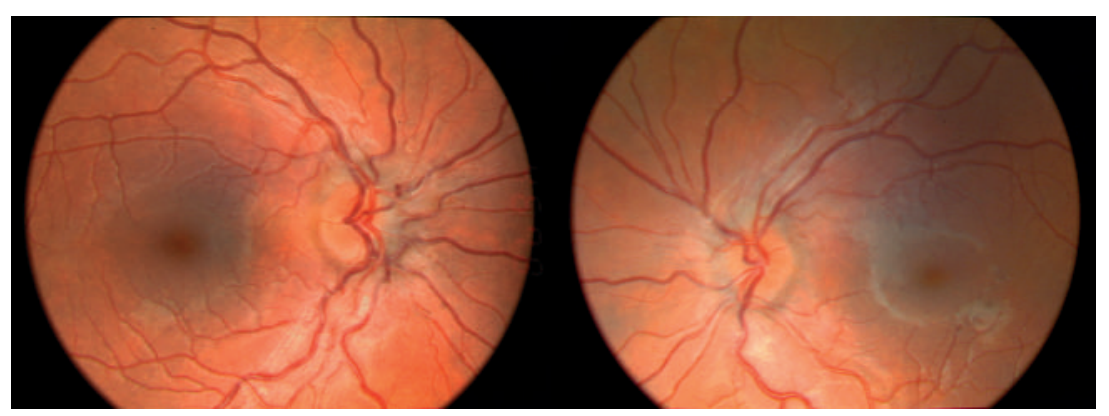

Figure 2 Examination 6 weeks later showed most of the haemorrhages and disc swelling had cleared.

to the ophthalmology department for assessment of his disc swelling.

He had been seen in the eye clinic a year before this presentation complaining of coloured lines in his field of vision. No abnormality was found and his discs on that occasion were noted to be normal. On examination this time he was noticed to have dysmorphic features, notably palpebral fissures slanting medially upwards, and abnormally formed low set ears. Visual acuities were $6 / 5$ in both eyes, colour vision testing was unremarkable, and there was no relative afferent pupillary defect. There was no sign of cataract formation or any other abnormality of the anterior segments. Funduscopy revealed bilateral disc swelling with extensive peripapillary haemorrhages in both eyes (fig 1). The diagnosis of hypoparathyroidism combined with the patient's abnormal facies raised the suspicion of a genetic disorder and blood was then sent for chromosomal analysis. His hypoparathroidism was treated with vitamin D and calcium supplements and he responded well with his calcium reaching normal levels within a few days. Examination 6 weeks after his calcium had normalised showed most of the haemorrhages and disc swelling had cleared (fig 2). Results of chromosomal testing revealed a region 11.2 microdeletion of the long arm (q) of chromosome 22. Other members of his family are now also being tested.

\section{Comment}

Chromosome 22q11.2 deletion syndrome is one of the more common causes of congenital and childhood hypoparathyroidism which can lead to hypocalcaemia. Hypocalcaemia is a known cause of disc swelling, ${ }^{4}$ the mechanism of which is not known. Some patients with hypocalcaemic disc swelling have a loss of acuity and field typical of an 
Table 1 Mean change in pupillary diameter in patients with Horner's syndrome

\begin{tabular}{|c|c|c|c|c|c|c|c|c|}
\hline & \multirow{2}{*}{\multicolumn{2}{|c|}{ Baseline pupil }} & \multicolumn{2}{|c|}{$\begin{array}{l}\text { Change in pupil diameter } \\
\text { following }\end{array}$} & \multicolumn{2}{|c|}{$\begin{array}{l}\text { Change in pupil diameter } \\
\text { following }\end{array}$} & \multicolumn{2}{|c|}{$\begin{array}{l}\text { Change in pupil diameter } \\
\text { following }\end{array}$} \\
\hline & & & \multicolumn{2}{|l|}{ Cocaine } & \multicolumn{2}{|c|}{ Hyrdoxyamphetamine } & \multicolumn{2}{|l|}{ Phenylephrine } \\
\hline & \multicolumn{2}{|l|}{ Size $(\mathrm{mm})$} & \multicolumn{2}{|l|}{$10 \%(\mathrm{~mm})$} & \multicolumn{2}{|l|}{$1 \%(\mathrm{~mm})$} & \multicolumn{2}{|l|}{$1 \%(\mathrm{~mm})$} \\
\hline & Normal pupil & Horner's pupil & Normal pupil & Horner's pupil & Normal pupil & Horner's pupil & Normal pupil & Horner's pupil \\
\hline Central $(n=2)$ & 3.25 & 2 & 1.5 & 0.25 & 2.1 & 2.25 & 0.3 & 0.25 \\
\hline $\begin{array}{l}\text { Pre-ganglionic } \\
(n=1)\end{array}$ & 4 & 2.5 & 3.0 & 0.5 & 2.5 & 2.9 & 0.2 & 0.5 \\
\hline $\begin{array}{l}\text { Post-ganglionic } \\
(\mathrm{n}=11)\end{array}$ & 4.0 & 2.9 & 1.8 & 0.2 & 1.8 & 0.3 & 0.2 & 1.9 \\
\hline
\end{tabular}

of a patient with central (first order) Horner's syndrome should not dilate, while a preganglionic (second order) pupil may dilate minimally'; a normal pupil may, at best, dilate minimally. The purpose of this study was to compare the pupillary response of patients with Horner's syndrome to phenylephrine $1 \%$ and hydroxyamphetamine $1 \%$.

Fourteen consecutive patients with Horner's syndrome were prospectively tested with cocaine $10 \%$, hydroxyamphetamine $1 \%$, and phenylephrine $1 \%$ on separate days, at least 3 days apart. All pupils were measured in the same room lighting with a standard ruler 1 hour after instillation of two drops. Phenylephrine $1 \%$ was prepared on each examination day by diluting phenylephrine $10 \%$ hydrochloride $(0.1 \mathrm{ml})$ with $0.9 \mathrm{ml}$ of preservative free natural tear drops. The clinician interpreting each pharmacological test was masked to the results of the other pharmacological tests and the cause of Horner's syndrome.

Nine of the 14 patients were men. The average age was 59 years (range 34-74 years). All patients underwent magnetic resonance imaging of head and neck, magnetic resonance angiography of the neck, and computed tomography of the chest. Horner's syndrome was considered to be central in two patients and pre-ganglionic in one, based on neuroimaging. All 11 patients diagnosed with postganglionic Horner's syndrome had normal neuroimaging, an isolated Horner's syndrome, positive cocaine test and positive hydroxyamphetamine test. Table 1 shows the baseline pupil size and the change with each of the three pharmacological tests. After pharmacological testing with phenylephrine $1 \%$, the mean increase in pupil size in patients with postganglionic Horner's syndrome was $2.3 \mathrm{~mm}$ (SD $1.1 \mathrm{~mm}$ ) and in the contralateral normal pupil was $0.2 \mathrm{~mm}$ (SD $0.2 \mathrm{~mm}$ ) (paired $t$ test: $\mathrm{p}<0.0001$ ). The sensitivity of phenylephrine $1 \%$ was $81 \%$ and the specificity $100 \%$. The mean increase in pupil diameter on the affected side in patients with postganglionic Horner's syndrome after hydroxyamphetamine $1 \%$ was $0.27 \mathrm{~mm}$ (SD $0.3 \mathrm{~mm}$ ) and was $2.65 \mathrm{~mm}$ (SD 0.3 ) in the contralateral normal pupil.

\section{Comment}

The law of denervation supersensitivity states that an organ deprived of its normal innervation becomes more sensitive to the chemical transmitter normally released from those nerves. Thompson and Mensher documented supersensitivity of the iris dilator to phenylephrine $1 \%$ in one patient. ${ }^{1}$ They further tested 13 patients but used a $10 \%$ concentration, which dilates the normal pupil. They determined that the affected pupil of the three patients with post-ganglionic lesions dilated sooner and more vigorously than the unaffected pupil. Ramsay tested 14 patients with phenylephrine $1 \%$ and found in patients with Horner's syndrome that $71 \%$ of pupils were supersensitive. However, the responses of the postganglionic and non-post-ganglionic Horner's syndrome were not reported separately. Other studies have reported on the use of phenylephrine $10 \%^{1}$ and epinephrine $1 \%$, and adrenaline (epinephrine) $0.1 \%^{1}$ in the pharmacological testing of Horner's, but none has investigated the efficacy of phenylephrine $1 \%$ in identifying post-ganglionic Horner's lesion. Our study shows that phenylephrine dilates the post-ganglionic Horner's pupil, but not the non-post-ganglionic or normal pupil. We found the sensitivity of $81 \%$ and a specificity of $100 \%$. Hydroxyamphetamine $1 \%$ has been shown to have a sensitivity of $93 \%$ and specificity of $83 \%{ }^{4}$ Only one out of 11 patients with Horner's syndrome in our series had a lesion, which localised to the postganglionic neuron with hydroxyamphetamine $1 \%$, but not with phenylephrine $1 \%$.

Phenylephrine $1 \%$ has some limitations:

(1) It does not dilate the normal pupil. If neither pupil dilates with phenylephrine $1 \%$ it could be either because the lesion is nonpost-ganglionic or the drops are ineffective.

(2) The degree of supersensitivity is determined by the extent of denervation. A partial post-ganglionic lesion may be difficult to distinguished from a preganglionic lesion; in both conditions the iris may dilate minimally. In our study there was only one patient with a preganglionic lesion.

(3) Tests for supersensitivity may also be subject to false positive errors owing to the variations in penetration of the drug. The drug should be placed strictly on intact corneas so that the same dose reaches each iris.

(4) Supersensitivity of the iris dilator increases with age. Phenylephrine sensitivity of the iris increases by $0.23 \mathrm{~mm}$ per decade after age 20 .

In summary, we here report the first series of patients with Horner's syndrome, which compared the pupillary response of phenylephrine $1 \%$ to hydroxyamphetamine $1 \%$. Phenylephrine $1 \%$ correlates well with the results of hydroxyamphetamine $1 \%$ in localising the lesion to the post-ganglionic neuron and is a reliable alternative to hydroxyamphetamine $1 \%$ should pharmacological testing be desired and hydroxyamphetamine $1 \%$ not be available.

H V Danesh-Meyer Deparment of Ophthalmology, University of Auckland, Auckland, New Zealand

H V Danesh-Meyer, P Savino, R Sergott

Neuro-ophthalmic Services, Wills Eye Hospital, Thomas Jefferson Medical School, Philadelphia, PA,

USÁ

Correspondence to: Associate professor Helen V Danesh-Meyer Department of Ophthalmology, University of Auckland, Private Bag 92019, Auckland, New Zealand; h.daneshmeyer@auckland.ac.mz

Accepted for publication 25 July 2003

\section{References}

1 Thompson HS, Mensher JH. Adrenergic mydriasis in Horner's syndrome. Am J Ophthalmol 1971;72:472-80.

2 Ramsay DA. Dilute solutions of phenylephrine and pilocarpine in the diagnosis of disordered autonomic innervation of the iris. J Neurol Sci 1986;73:125-34.

3 Maloney WF, Younge BR, Moyer NJ. Evaluation of the causes and accuracy of pharmacologic localization Horner's syndrome. Am J Ophthalmol 1980;90:394-402.

4 Cremer SA, Thompson S, Digree KB, et al. Hydroxyamphetamine mydriasis in Horner's syndrome. Am J Ophthalmol 1990;1 10:71-6.

\section{Tetraspanin protein KAIl expres- sion in retinoblastoma}

$\mathrm{KAIl} / \mathrm{CD} 82$ is a metastasis suppressor gene located on human chromosome $11 \mathrm{pl} 1.2$. It is a member of the structurally distinct family of cell surface glycoprotein, transmembrane 4-protein superfamily. ${ }^{1}$ KAIl was initially isolated as a gene that suppressed metastasis of rat prostate tumour cells. ${ }^{2}$ KAIl is downregulated in several types of human malignancies. ${ }^{3-5}$ The purpose of this study was to investigate the expression of KAIl in retinoblastoma and to correlate clinicopathologically.

\section{Methods}

There were 30 archival specimens of retinoblastomas from 2000 to 2002 . There were 12 tumours with no invasion of choroid, or optic nerve and 18 tumours with invasion of choroid/optic nerve, and one tumour which had metastasised to the submandibular region. There were six well differentiated tumours, six moderately differentiated tumours, and 18 poorly differentiated tumours (table 1). Immunohistochemical 
Table 1 Clinicopathological and immunohistochemical features of the retinoblastomas used in the study

\begin{tabular}{|c|c|c|c|c|c|c|}
\hline \multirow[b]{2}{*}{ No } & \multirow{2}{*}{$\begin{array}{l}\text { Age } \\
\text { (years)/ } \\
\text { sex }\end{array}$} & \multirow[b]{2}{*}{$\begin{array}{l}\text { Clinicopathological } \\
\text { parameters }\end{array}$} & \multirow[b]{2}{*}{ Chemotherapy } & \multicolumn{2}{|c|}{$\begin{array}{l}\text { KAll } \\
\text { immunoreactivity }\end{array}$} & \multirow{2}{*}{$\begin{array}{l}\text { Ki67 } \\
\% \text { cells } \\
\text { stained }\end{array}$} \\
\hline & & & & $\begin{array}{l}\% \text { Cells } \\
\text { stained }\end{array}$ & $\begin{array}{l}\text { Stain } \\
\text { intensity }\end{array}$ & \\
\hline 1 & $2 / M$ & Both eyes, (RE) well diff & No & 90 & $3+$ & 40 \\
\hline 2 & $5 / M$ & LE, poor diff & No & 90 & $3+$ & 37 \\
\hline 3 & $1 / F$ & RE, well diff & No & 90 & $3+$ & 63 \\
\hline 4 & $2 / M$ & Both eyes, (RE), well diff & No & 50 & $2+$ & 40 \\
\hline 5 & $8 / M$ & $L E$, poor diff & No & 90 & $3+$ & 40 \\
\hline 6 & $4 / F$ & $\mathrm{LE}$, poor diff & No & 80 & $3+$ & 60 \\
\hline 7 & $1 / F$ & $\mathrm{LE}$, poor diff & No & 95 & $3+$ & 49 \\
\hline 8 & $5 / \mathrm{F}$ & RE, poor diff & No & 45 & $2+$ & 50 \\
\hline 9 & $1 / F$ & Both eyes, (RE), mod diff & No & 80 & $3+$ & 40 \\
\hline 10 & $2 / F$ & Both eyes, RE, mod diff & No & 85 & $3+$ & 70 \\
\hline 11 & $2 / \mathrm{F}$ & Both eyes, $L E$, mod diff & No & 40 & $2+$ & 66 \\
\hline 12 & $1.5 / \mathrm{M}$ & RE, well diff & No & 85 & $3+$ & 50 \\
\hline 13 & $7 / F$ & $\begin{array}{l}\text { LE, mod diff, No choroidal } \\
\text { invasion, surgical end of the } \\
\text { optic nerve invaded }\end{array}$ & $\begin{array}{l}\text { Postop chemo } 3 \\
\text { cycles }\end{array}$ & 1 & 0 & 56 \\
\hline 14 & $1 / M$ & RE, poor diff, Pre laminar & No & 20 & $1+$ & 60 \\
\hline 15 & $2 / F$ & $\begin{array}{l}\mathrm{RE} \text {, poor diff, diffuse choroidal } \\
\text { invasion, surgical end of optic } \\
\text { nerve invaded }\end{array}$ & $\begin{array}{l}\text { Postop chemo } 4 \\
\text { cycles }\end{array}$ & 1 & 0 & 70 \\
\hline 16 & $4 / M$ & Both eyes, LE, poor diff & $\begin{array}{l}\text { Postop chemo } 4 \\
\text { cycles }\end{array}$ & 1 & 0 & 70 \\
\hline 17 & $4 / \grave{F}$ & RE, mod diff, lamina cribrosa & No & 40 & $2+$ & 64 \\
\hline 18 & $2 / M$ & $\mathrm{RE}$, poor diff, lamina cribrosa & No & 20 & $1+$ & 66 \\
\hline 19 & $1.1 \mathrm{M} / \mathrm{F}$ & $\begin{array}{l}\mathrm{RE} \text {, poor diff, diffuse choroidal } \\
\text { invasion and post laminar optic } \\
\text { nerve invasion }\end{array}$ & $\begin{array}{l}\text { Postop chemo } 6 \\
\text { cycles }\end{array}$ & 1 & 0 & 80 \\
\hline 20 & $3 / M$ & $\begin{array}{l}\mathrm{LE} \text {, poor diff, post laminar } \\
\text { invasion }\end{array}$ & $\begin{array}{l}\text { Postop chemo } 2 \\
\text { cycles }\end{array}$ & 10 & $1+$ & 60 \\
\hline 21 & $4 / F$ & $\begin{array}{l}\mathrm{RE} \text {, mod diff, focal choroidal } \\
\text { invasion }\end{array}$ & No & 10 & $1+$ & 69 \\
\hline 22 & $2 / \mathrm{F}$ & RE, poor diff, post laminar & No & 0 & 0 & 70 \\
\hline 23 & $2.5 / \mathrm{F}$ & $R E$, poor diff, diffuse & No & 10 & $1+$ & 60 \\
\hline 24 & $3 / M$ & $R E$, poor diff, post laminar & No & 1 & 0 & 70 \\
\hline 25 & $5 / M$ & $\begin{array}{l}\mathrm{RE} \text {, poor diff, diffuse choroidal } \\
\text { invasion, surgical end of ON } \\
\text { invaded }\end{array}$ & $\begin{array}{l}\text { Postop chemo } 6 \\
\text { cycles }\end{array}$ & 1 & 0 & 60 \\
\hline 26 & $5 / F$ & $\begin{array}{l}\text { Both eyes,(RE) well diff, pre } \\
\text { laminar, focal choroidal } \\
\text { invasion }\end{array}$ & No & 10 & $1+$ & 75 \\
\hline 27 & $2 / F$ & Both eyes, (LE) poor diff, diffuse & No & 10 & $1+$ & 60 \\
\hline 28 & $2 / M$ & RE, poor diff, post laminar & No & 1 & 0 & 80 \\
\hline 29 & $1 / F$ & $\begin{array}{l}\text { Both eyes, (RE) well diff, lamina } \\
\text { cribrosa }\end{array}$ & $\begin{array}{l}\text { Preop and postop } \\
\text { chemo }\end{array}$ & 10 & $1+$ & 61 \\
\hline 30 & $2 / F$ & $\begin{array}{l}\text { Poor diff, diffuse choroidal, } \\
\text { post laminar, metastasis to } \\
\text { submandibular area }\end{array}$ & Postop chemo & 1 & 0 & 60 \\
\hline
\end{tabular}

staining was performed using a sensitive labelled streptavidin biotin (LSAB kit, Dako) on tumours using monoclonal antibodies for tetraspanin KAIl (C33, Novacastra) and for proliferation index Ki-67 (Clone MIB-1, Dako, Denmark) after antigen retrieval.

The immunoanalysis for KAIl was done based on the percentage of cells and the staining intensity. KAIl was scored after analysing 15 tumour fields and on a four tiered scale: 0 , negative $(0 \%-5 \%$ positive cells); $1+$, weak to moderate $(6 \%-30 \%) ; 2+$, intense $(31 \%-50 \%)$; and $3+$, very intense $(>50 \%)$. Labelling index for Ki 67 positive cells was expressed by counting a minimum of 500 cells in the staining area. Tumours were divided into two groups; group I $>50 \%$ of cells showing positive Ki 67 expression and group II $<50 \%$ of cells showing positive for Ki 67 . Data were analysed for statistical significance.

\section{Results}

The immunohistochemical details are given in table 1. KAIl expression was seen in the control lymphoid follicle of the tonsil (fig 1A). Intense KAIl positivity with more than $80 \%$ positivity was seen in all 12/12 tumours with no invasion (fig 1B). Among the 18/18 tumours with invasion, KAIl was decreased in all 18. The invading front of the tumour had less KAIl than the tumour at the central portion. Retinoblastomas with focal and diffuse invasion of choroid had negative KAIl immunoreactivity. Tumours with prelaminar optic nerve invasion had weak KAIl immunoreactivity (fig lC). Tumours at the post-laminar and surgical end of the optic nerve (inset, fig $\mathrm{IC}$ ) and at the metastatic site (fig 1D) had negative KAIl immunoreactivity. Negative KAil reactivity was seen in 50\% $(9 / 18)$ of poorly differentiated retinoblastomas. Statistically significant correlation was observed between KAil expression and Ki 67 labelling index in the whole study group $(\mathrm{p}<0.001)$. No statistically significant correlation was observed between KAIl expression and differentiation. Significant statistical correlation was observed when KAIl expression was compared with tumour invasion $(\mathrm{p}<0.001)$.

\section{Comment}

Retinoblastoma joins a growing list of cancers in which downregulation of KAIl is associated with tumour progression. In our study KAIl was identified by the monoclonal antibody CD33. ${ }^{6}$ It was originally shown as inhibitory to syncytium formation induced by human T cell leukaemia virus type I, and this specific inhibition to syncytium formation induced by some human $\mathrm{T}$ cell line by this antibody was strongly associated with altered glycosylation of cell surface antigen, suggesting that the $\mathrm{C} 33$ antigen-that is, KAIl, might have a possible role in the cell to cell adhesion mechanism. ${ }^{7}$

Thus, KAIl may link to the cell surface molecules, such as integrins, E-cadherin, and other TM4SF members, and loss of KAIl function may have a significant role in the progression of retinoblastoma. ${ }^{7}$ The mechanism of KAIl downregulation is not known. The $5^{\prime}$ promoter region of the gene contains a $\mathrm{CpG}$ island, ${ }^{8}$ raising the possibility of gene silencing by promoter methylation. Thus, biologically, our findings suggest a potential implication of KAIl in tumour progression and these molecules may provide novel insights into tumour progression in retinoblastoma.

\section{Acknowledgement}

Financial support: Indian Council Of Medical Research, India.

S AmirthaLakshmi, V Pushparaj, V Krishnamurthy, J Biswas, S Krishnakumar Department of Ocular Pathology, Medical and Vision Research Foundation, Sankara Nethralaya, Chennai,

$$
\text { India }
$$

M P Shanmugam

Department of Ocular Oncology, Medical and Vision Research Foundation, Sankara Nethralaya, Chennai, India

Correspondence to: Dr Subramanian Krishnakumar, Vision Research Foundation, Sankara Nethralaya, 18 College Road, Chennai 600 006, Tamil Nadu, India; drkrishnakumar_2000@yahoo.com

Accepted for publication 1 August 2003

Proprietary interest: The authors have no financial interest in any of the materials used in the study.

\section{References}

1 Maecker HT, Todd SC, Levy S. The tetraspanin superfamily: molecular facilitators. Faseb J 1997; 11:428-42

2 Dong JT, Lamb PW, Rinker-Schaeffer CW, et al. Kail, a metastasis suppressor gene for prostate cancer on human chromosome $11 \mathrm{pl} 1$. 2. Science (Washington DC) 1995;268:884-6.

3 Yu Y, Yang J-L, Markovic B, et al. Loss of KAII messenger RNA expression in both high-grade and invasive human bladder cancers. Clin Cancer Res 1997; 3:1045-9.

4 Huang C I, Kohno N, Ogawa E, et al. Correlation of reduction in $\mathrm{mrp}-1 / \mathrm{cd} 9$ and KAll/cd82 expression with recurrences in breast cancer patients. Am J Pathol 1998; 153:973-83.

5 Guo X, Friess H, Graber HU, et al. KAll expression is up-regulated in early pancreatic cancer and decreased in the presence of metastases. Cancer Res 1996;56:4876-80.

6 Imai T, Fukudome K, Takagi S, et al. C33 antigen recognized by monoclonal antibodies inhibitory to human T cell leukemia virus type 1-induced 
syncytium formation is a member of a new family of transmembrane proteins including $\mathrm{cd} 9, \mathrm{~cd} 37$, cd53, and cd63. J Immunol 1992; 149:2879-86.

7 Rubinstein E, Boucheix C. Tetraspans. In: Kreis T, Vale R, eds. Guidebook to the extracellular matrix and adhesion proteins. Oxford: Oxford University Press, 1999:321-4.

8 Dong JT, Isaacs WB, Barrett JC, et al. Genomic organization of the human KAll metastasissuppressor gene. Genomics 1997;41:25-32.
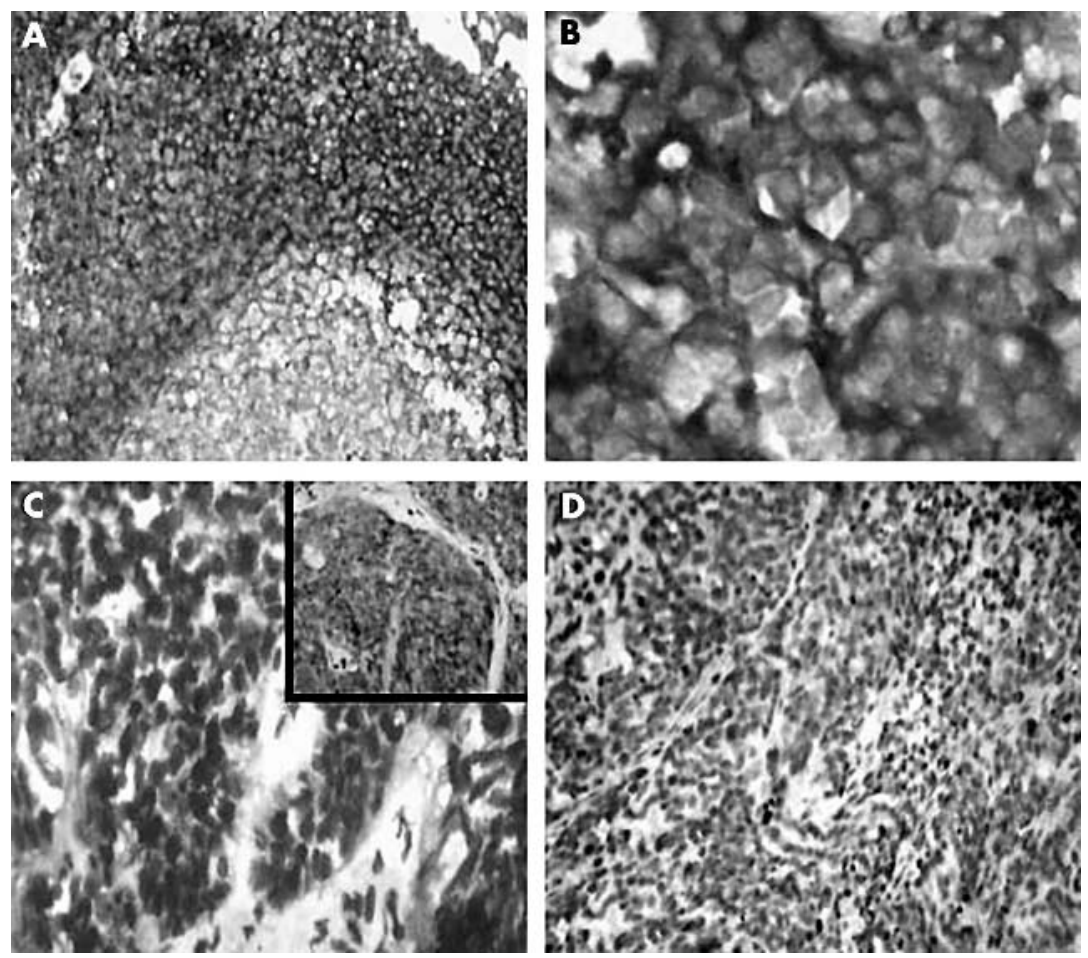

Figure 1 (A) Microphotograph showing the KAIl expression in control lymphoid follicle of the tonsil (magnification $\times 200$ ). (B) Microphotograph showing the intense membrane immunoreactivity of KAIl in retinoblastoma with no invasion (magnification $\times 200$ ). (C) Microphotograph showing the weak KAll immunoreactivity of tumour cells invading the lamina cribrosa of the optic nerve (magnification $\times 100$ ). Inset shows negative KAll in tumour cells at the surgical end of optic nerve. (D) Microphotograph showing the negative KAll immunoreactivity of tumour cells at the metastatic site in the submandibular region (magnification $\times 200$ ).

\section{Bilateral ocular surface squamous neoplasia: a clinicopathological case report}

Ocular surface squamous neoplasia (OSSN) was first described by Lee and Hirst ${ }^{1}$ as an umbrella term that encompasses intraepithelial and invasive squamous cell carcinoma of the conjunctiva and cornea. The incidence of OSSN ranges from 0.02 to 3.5 per 100000 population and varies geographically, with greater frequency near the equator. Generally, it is a slow growing tumour that rarely metastasises, but is capable of causing extensive local tissue destruction. Bilateral OSSN is extremely rare ${ }^{2-7}$ and offers a unique opportunity to study the biological characteristics of bilateral OSSN of the conjunctiva. The following case report describes the clinical presentation, histopathology, and immunohistochemical evaluation of tumour proliferation markers of a patient diagnosed with bilateral OSSN.

\section{Case report}

An 86 year old white woman was referred to the Doheny Eye Institute because of redness in her right eye that had developed over a period of several months. She had undergone a mastectomy in 1954. She had no history of ocular trauma, toxin exposure, or tobacco use. Her brother and sister died from liver cancer. An ophthalmic examination revealed a visual acuity of $20 / 100$ in each eye. Ectropion and indurated lower eyelid margins were present bilaterally with no loss of cilia. A closer examination revealed a thickened epithelium that lined the palpebral conjunctiva and cul del sac of the right eye (fig 1A). The left lower palpebral conjunctiva showed similar changes. However, there was a focal nodule on the inferior bulbar conjunctiva (fig lB).

The patient underwent a biopsy of the right palpebral conjunctiva. Histopathological examination of the specimen revealed
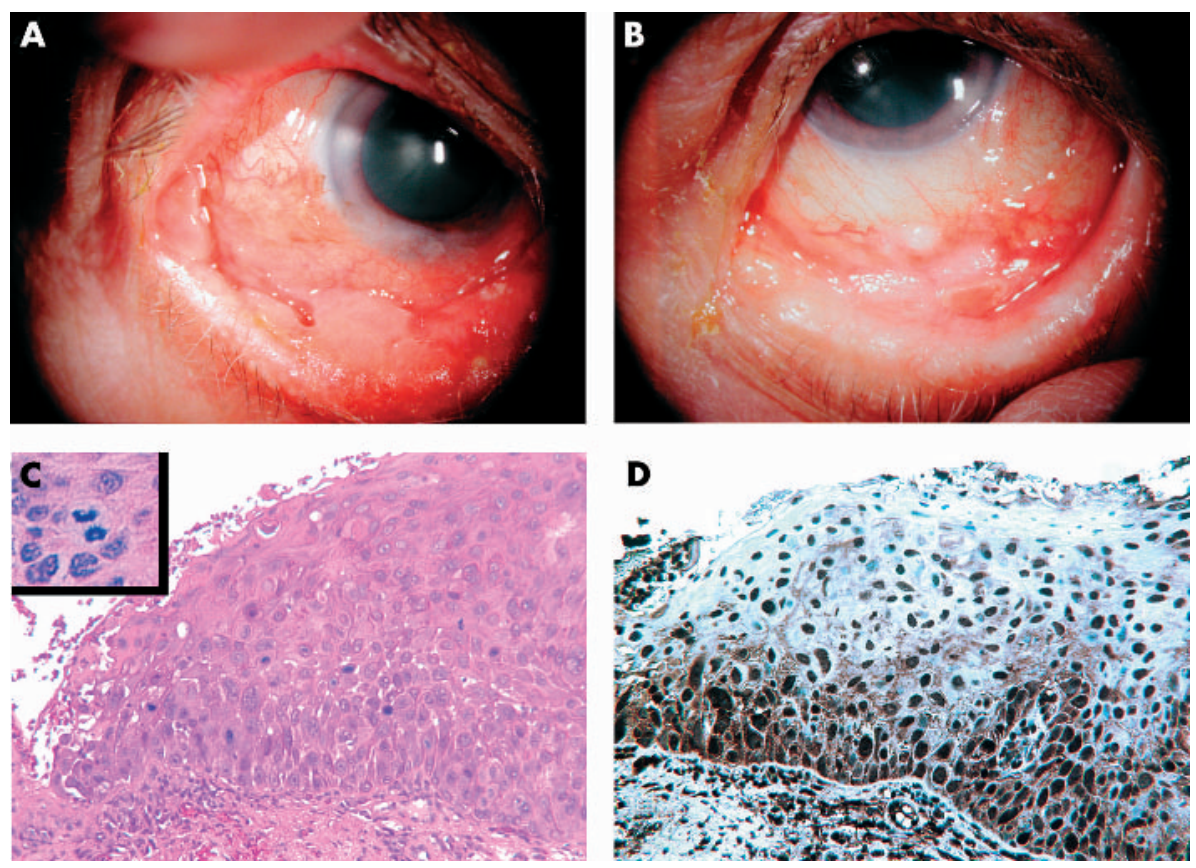

Figure 1 (A) Clinical photograph of the right eye showing diffuse thickened epithelium involving the palpebral conjunctiva and cul de sac. (B) Clinical photograph of the left eye showing irregular thickened epithelium and a focal mass noted on the inferior bulbar conjunctiva. (C) Photomicrograph from the left conjunctival biopsy showing squamous cell carcinoma in situ. Note that multiple abnormal mitotic figures (inset) are present (haematoxylin and eosin; original magnification $\times 250$; inset $\times 325$ ). (D) Immunohistochemistry for human papillomavirus (HPV) showing positive staining in majority of the tumour cells (original magnification $\times 250$ ). 
Table 1 Comparative immunohistochemical findings

\begin{tabular}{lll}
\hline \multicolumn{2}{c}{ Right conjunctival biopsy } & \multicolumn{1}{c}{ Left conjunctival biopsy } \\
\hline Immunohistochemical marker & \\
Pankeratin & ++++ & ++++ \\
HPV & ++++ & ++++ \\
P53 & ++++ & - \\
Bcl-2 & - & ++ \\
MIB-1 & +++ & \\
\hline &
\end{tabular}

acanthotic epithelium with squamous metaplasia, occasional dyskeratotic cells, parakeratosis, and hyperkeratosis. Multiple abnormal mitotic figures were present. The basement membrane was intact, and a diagnosis of conjunctival squamous cell carcinoma in situ was diagnosed. A subsequent biopsy of the left conjunctiva revealed full thickness squamous metaplasia of the epithelium with acanthotic and marked dysplastic changes. Multiple abnormal mitotic figures were seen and the basement membrane was also intact, with an extensive chronic inflammatory cell infiltrate in the stroma (fig $\mathrm{lC}$ ). These findings were also consistent with a diagnosis of squamous cell carcinoma in situ of the conjunctiva.

Immunohistochemical analysis (Dako, Carpinteria, CA, USA) revealed that neoplastic cells were positive for pankeratin, human papillomavirus (HPV) (fig 1D), and Ki-67 (MIB-1) in both specimens. Moreover, both biopsies indicated the presence of a few bcl-2 positive cells. The right eye biopsy was p53 positive and the left was p53 negative. The immunohistochemically positive cells were counted by methods described previously. ${ }^{8}$ Table 1 summarises the immunohistochemical findings of both biopsies. Because of the patient's fragile health, surgical intervention was deferred and she was treated with topical mitomycin $\mathrm{C}$ in both eyes. At the follow up examination 13 months after the biopsy, a mass was found in the right lower palpebral conjunctiva, but there was no evidence of such lesion in the left conjunctiva or metastasis.

\section{Comment}

The aetiology of bilateral OSSN remains unclear. Causative factors that are believed to contribute to the development of unilateral OSSN include ultraviolet light exposure, ocular trauma, predisposing genetic factors, and infection with HPV. Previous reports have provided convincing evidence of an association with HPV type 16 in some cases of bilateral conjunctival dysplasia. ${ }^{3}$ It has been postulated that the interaction between HPV and ultraviolet light may have a role in the development of HPV related tumours in patients who are exposed to the sun. ${ }^{9}$ However, both conjunctival lesions in the present case were located in areas that were not exposed to sunlight, suggesting a possibility that HPV infection in both eyes may have led to the development of bilateral OSSN.

Conjunctival OSSN has been described as a slow growing, well differentiated tumour of low grade malignancy that responds to local excision and rarely metastasises. Immunohistochemical studies of bilateral lesions allow comparison of the proliferative potency in both eyes, and offer a unique opportunity to study some biological aspects of bilateral tumours under the same environmental conditions at the same point in time. In recent years p53, bcl-2, and MIB- 1 have been used as markers of proliferative potency. ${ }^{8} 10$ The p53 gene is a common cellular target in human carcinogenesis and is thought to have an important role in cellular proliferation. In contrast with the wild type p53, mutants of the p53 gene produce an abnormal protein with a long half life and are thus immunohistochemically detectable. Also, p53 has been reported to be a prognostic marker in several tumours. ${ }^{8}{ }^{10} \mathrm{Bcl}-2$ is a proto-oncogene that is thought to have a role in oncogenesis by inhibiting programmed cell death and preserving cells from p53 induced apoptosis. ${ }^{8}$ However, the mutant p53 protein also induces apoptosis and decreases the expression of bcl-2 proteins. Mahomed et $\mathrm{al}^{8}$ suggested that the interplay between the effects of the increased mutant p 53 proteins and the absence of bcl-2 expression in tumorigenesis may promote clonal expansion, leading to progressively increased genomic instability. The synergy of the presence of mutant p53 and absence of bcl2 in the present case might have allowed the progression of the tumour in the right conjunctiva.

Ki-67 is a nuclear antigen expressed in all stages of the cell cycle except the resting stage. MIB-1 is a monoclonal antibody that recognises the Ki-67 antigen, which is a marker of cellular proliferation and reported to be a prognostic factor for various cancers. The high immunoreactivity of MIB-1 in conjunctival OSSN is usually associated with highly aggressive tumour growth. ${ }^{8}$ Our results demonstrate a higher immunoreactivity of MIB-1 in the right conjunctival specimen. These findings indicate that the right conjunctival specimen is more aggressive than the left, and is consistent with this patient's clinical course.

In conclusion, this case represents a rare example of conjunctival pathology: OSSN as a bilateral tumour. To our knowledge, this is also the first report that compares the right and left biopsies of conjunctival OSSN by immunohistochemical analysis of potential oncogenic factors. Enhanced expression of MIB- 1 and presence of mutant $\mathrm{p} 53$ protein in the absence of bcl-2 may contribute to the aggressive biology of OSSN.

\section{Acknowledgements}

This work was supported in part by NEI core grant Ey03040 and by an unrestricted grant from Research to Prevent Blindness Inc, New York, USA.

Y Usui

Department of Ophthalmology, Tokyo Medica University, Tokyo, Japan
Y Usui, G O Waring, R F See, N A Rao A Ray Irvine Ocular Pathology Laboratory, Keck School of Medicine of the University of Southern California, Los Angeles, CA, USA

R F See, N A Rao

Doheny Eye Institute, Keck School of Medicine of the University of Southern California, Los Angeles, CA,

N A Rao

Department of Ophthalmology, Keck School of Medicine of the University of Southern California, Los Angeles, CA, USA

A C Marrone

Department of Ophthalmology, Torrance Memorial Medical Center, Torrance, CA, USA

Correspondence to: Yoshihiko Usui, MD, Department of Ophthalmology, Tokyo Medical University, 6-7-1 Nishi-shinjuku, Shinjuku-ku, Tokyo 160-0023, Japan; usuyoshi@aol.com

Accepted for publication 1 August 2003

\section{References}

1 Lee GA Hirst LW. Ocular surface squamous neoplasia. Surv Ophthalmol 1995;39:429-50.

2 Cervantes G, Rodriguez AA Jr, Leal AG. Squamous cell carcinoma of the conjunctiva: clinicopathological features in 287 cases. Can J Ophthalmol 2002;37:14-19.

3 Odrich MG, Jakobiec FA, Lancaster WD, et al. A spectrum of bilateral squamous conjunctival tumors associated with human papillomavirus type 16. Ophthalmology 1991:98:628-35.

4 Tritten JJ, Beati D, Sahli R, et al. [Bilateral conjunctivo-palpebral tumor in an immunocompetent man caused by human papilloma virus.] Klin Monatsbl Augenheilkd 1994:204:453-5.

5 Olurin O. Bilateral conjunctival epithelioma in an adolescent. Ann Ophthalmol 1971;3:633-6.

6 Lam A, Borzeix A, Seck CM, et al. [A familial form of epidermoid carcinoma of the conjunctiva.] J Fr Ophtalmol 1996; 19:143-8.

7 Tabin G, Levin S, Snibson G, et al. Late recurrences and the necessity for long-term follow-up in corneal and conjunctival intraepithelial neoplasia. Ophthalmology 1997;104:485-92.

8 Mahomed A, Chetty R. Human immunodeficiency virus infection, $\mathrm{Bcl}-2$, p53 protein, and $\mathrm{Ki}-67$ analysis in ocular surface squamous neoplasia. Arch Ophthalmol 2002;120:554-8.

9 Arends MJ, Wyllie AH, Bird CC. Papillomaviruses and human cancer. Hum Pathol 1990;21:686-98.

10 Aoki S, Kubo E, Nakamura S, et al. Possible prognostic markers in conjunctival dysplasia and squamous cell carcinoma. Jpn J Ophthalmol 1998;42:256-61.

\section{Employing endoscopic guidance for placement of a black diaphragm aniridia intraocular lens following destructive Acanthamoeba sclerokeratitis}

Anterior segment reconstruction can be particularly challenging when anatomic landmarks are lost. We describe a case of destructive Acanthamoeba sclerokeratitis resulting in aniridia, aphakia, loss of limbal architecture, and corneal opacification that was approached surgically with penetrating keratoplasty and placement of a black diaphragm aniridia intraocular lens under endoscopic guidance.

\section{Case report}

Our patient, a 48 year old female contact lens wearer, was diagnosed with Acanthamoeba keratitis in June 2000. Before our evaluation, she had been treated with tobramycin and 
dexamethasone ointment, topical trifluridine, oral acyclovir, oral prednisone, and topical prednisolone acetate $1 \%$. We diagnosed Acanthamoeba keratitis and began aggressive treatment with polyhexamethyl biguanide, chlorhexidine, and oral clotrimazole. By January 2001, she was culture negative, but had developed necrotising sclerokeratitis with limbal involvement, dense corneal opacification, and descemetocoele formation. Urgent penetrating limbal keratoplasty was performed. Upon placement of the lid speculum, spontaneous perforation of the cornea occurred with prolapse of the lens and necrotic iris. We performed a $12.5 \mathrm{~mm}$ diameter keratolimbal resection, removed residual lens and necrotic iris, and performed anterior vitrectomy. A $13.0 \mathrm{~mm}$ keratolimbal graft was placed and covered with an amniotic membrane graft. (fig 1 )

Eighteen months later, the patient had negative cultures, a quiet eye, an opaque corneal graft, controlled intraocular pressure, and counting fingers vision with projection to all four quadrants. However, the patient complained of severe glare and light sensitivity. Soft contact lens wear was unsuccessful because of irregular postsurgical topography. After extensive discussion, this highly motivated patient elected to pursue further anterior segment reconstructive surgery to address the aniridia, aphakia in the absence of capsular support, and corneal opacity.

Penetrating keratoplasty and implantation of a sulcus fixated Morcher 67F black diaphragm polymethylmethacrylate lens was planned. External landmarks for transscleral suture fixation had been lost due to infectious necrosis and the large keratolimbal graft. Instead, suture placement was guided by an ocular endoscope (URAM E2 MicroProbe Laser System, EndoOptiks, New Jersey, USA). Following excision of an $8.0 \mathrm{~mm}$ diameter corneal button, a $10-0$ prolene suture on an STC-6 needle (Ethicon Inc, New Jersey, USA) was passed externally under a scleral flap and viewed internally via the endoscope as it entered the ciliary sulcus. The suture was passed through the lens fixation loop. A 25 gauge needle was passed externally into the ciliary sulcus under endoscopic visualisation, the STC- 6 needle was passed into its bore, and the complex guided out of the eye. This process was repeated for the opposing haptic, the sutures were tied, and an $8.0 \mathrm{~mm}$ donor button was placed. In the early postoperative period, the intraocular lens was positioned without obvious decentration or tilt, and the patient reported substantial improvement in her glare symptoms.

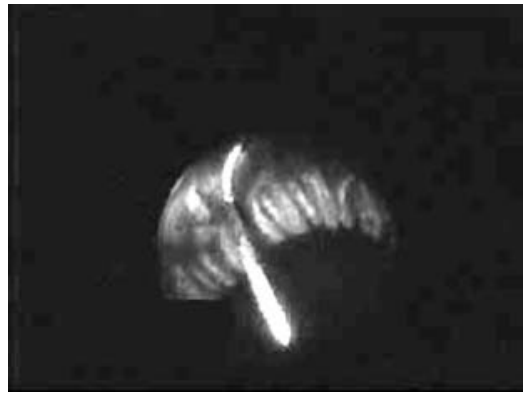

Figure 1 Endoscopic view of the STC-6 needle as it enters the ciliary sulcus.

\section{Comment}

A black diaphragm intraocular lens design allows simultaneous treatment of aniridia and aphakia. ${ }^{1}$ The Morcher $67 \mathrm{~F}$ has a $13.5 \mathrm{~mm}$ length, $10 \mathrm{~mm}$ diameter optic, and a $5 \mathrm{~mm}$ central clear zone. Precise haptic capture in the ciliary sulcus is necessary to minimise risks of haptic-optic crowding, mechanical irritation, and tilt or decentration of a small optic zone. Unfortunately, lens decentration and tilt is commonly observed following transscleral fixation of lenses. ${ }^{2}$ This can be attributed to suboptimal haptic position following blind passage of fixation sutures. ${ }^{3}$ Althaus and Sundmacher have described the usefulness of direct endoscopic visualisation in eyes undergoing transscleral suture lens fixation. ${ }^{4}$ In our patient, accurate lens position was critical, and the risk of lens malposition high, given her unfavourable anatomy. Our experience confirms that endoscopic visualisation is valuable for the placement of transscleral lens fixation sutures, particularly when surgical landmarks are lost and when mild lens malposition might adversely affect the surgical outcome.

A M Poothullil, S D McLeod, S Lin Department of Ophthalmology, University of California San Francisco, San Francisco, CA, USA

S D Mcleod

The Francis I Proctor Foundation University of California San Francisco, San Francisco, CA, USA

Correspondence to: Dr S D McLeod, Department of Ophthalmology, 10 Koret Way, K-301, San Francisco, CA 94143, USA; smcleod@itsa.ucsf.edu

Accepted for publication 4 August 2003

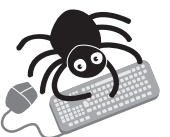

A video clip of this procedure is available on the BJO website (www.bjophthalmol.com)

\section{References}

1 Thompson CG, Fawzy K, Gryce IG, et al. Implantation of a black diaphragm intraocular lens for traumatic aniridia. $J$ Cataract Refract Surg 1999;25:808-13

2 Schein, et al. A randomized trial of intraocular lens fixation techniques with penetrating keratoplasty. Ophthalmology

1993; 100:1437-43

3 Hayashi K, Hayashi H, Nakao F, et al. Intraocular lens tilt and decentration, anterior chamber depth, and refractive error after trans-scleral suture fixation surgery. Ophthalmology 1999; 106:878-82.

4 Althaus C, Sundmacher R. Endoscopically controlled optimization of trans-scleral suture fixation of posterior chamber lenses in the ciliary sulcus. Ophthalmologe 1993;90:317-24.

\section{MAILBOX}

\section{Variant CJD and tonometry}

We read with interest the paper by Lim et al. The authors interestingly showed the importance of tonometer head wiping in reducing corneal epithelial cell count and the significance of damaged prism surfaces in trapping debris. They hypothesised the possible risk of variant Creutzfeld-Jakob disease (vCJD) transmission from cornea epithelial cells present on the tonometer surface. The question is really one of what constitutes the infectious dose of VCJD for this mode of transmission, and this is currently unknown.

There has been one definite, one probable, and two possible cases of CJD through corneal transplantation but one can hardly compare the prion load in a full thickness corneal graft with a mean epithelial cell count of 9/10 (after wiping or wipe/Milton). Two of the four cases of transmission had had multiple graft procedures.

The evidence from animal studies on CJD infected corneal transmission is also variable. Herzberg transplanted CJD infected corneas onto two Capuchin monkeys; both remained disease free for up to 4 years. ${ }^{2}$ Manuelidis et al showed transmission of CJD when infected corneas were placed directly into the anterior chamber of uninfected guinea pigs ${ }^{3}$ but did not show transmission of CJD after penetrating keratoplasty. Tateishi injected emulsified CJD infected cornea into the brains of six mice and only one developed characteristic changes after 2.8 years. ${ }^{4}$

These studies certainly suggest that an intraocular/intracerebral delivery must be needed for transmission but even then, the inocula that produced disease after intracerebral inoculation only irregularly transmit disease after peripheral (intraocular) inoculation. $^{5}$ In addition, host genetic factors (homozygosity at codon 129) will also determine the risk of susceptibility. ${ }^{6}$ There is also convincing evidence that the agent strain and host genotype will determine whether ocular involvement even occurs in experimental rodent models of scrapie. ${ }^{7}$

More recently, western blot analysis on eyes of patients that had been infected with sporadic CJD and $\mathrm{vCJD}^{8}$ confirmed earlier results (also in human eyes) that PrPSc could only be detected in the retina and not in cornea or sclera. From the evidence, the risk of transmission from tonometry would suggest this to be more theoretical than practical, and may be reduced further-as the authors suggest-by adequate wiping or regular replacement of tonometer prisms. The question of risk can only be truly resolved by transmission studies using primary human diseased eye tissue.

J S Mehta, R J Osborne, P A Bloom The Western Eye Hospital, London, UK

Correspondence to: Dr J S Mehta, 9 Sandringham Court, King \& Queen Wharf, Rotherhithe Street, London SE16 5SQ, UK; jodmehta@hotmail.com Accepted for publication 10 July 2003

\section{References}

1 Lim R, Dhillon B, Kurian KM, et al. Retention of corneal epithelial cells following Goldmann tonometry: implications for CJD risk. Br J Ophthalmol 2003;87:583-6.

2 Herzberg L. Creutzfeldt-Jakob disease and corneal grafts. Med J Aust 1979;1:248.

3 Manuelidis EE, Angelo JN, Gorgacz EJ, et al. Experimental Creutzfeldt-Jakob disease transmitted via the eye with infected cornea. N Engl J Med 1977;296:1334-6.

4 Tateishi J. Transmission of Creutzfeldt-Jakob disease from human blood and urine into mice. Lancet 1985:2:1074.

5 Brown P, Gibbs DJ, Rodgera-Johnson P, et al. Human spongiform encephalopathy: the National Institutes of Health series of 300 cases of experimental transmitted disease. Ann Neurol 1994:35:513-29. 
6 Mehta JS, Franks WA. The sclera, the prion, and the ophthalmologist. Br J Ophthalmol 2002;86:587-92

7 Foster JD, Fraser H, Bruce ME. Retinopathy in mice with experimental scrapie. Neuropathol Appl Neurobiol 1986; 12:185-96.

8 Head MW, Northcott V, Rennison K, et al. Prion protein accumulation in eyes of patients with sporadic and variant CJD. Invest Ophthalmol Vis Sci 2003;44:342-6.

\section{Intravitreal triamcinolone acetonide for exudative age related macular degeneration}

We read the article by Jonas et al on intravitreal triamcinolone injections for exudative age related macular degeneration with interest. ${ }^{1}$ The paper stated that visual acuity increased significantly $(\mathrm{p}<0.001)$ from 0.16 (SD 0.11 ) to a mean maximum of $0.23(0.17)$. The authors therefore picked the best from one of up to 10 postoperative visual acuity measurements and compared it with a single preoperative visual acuity measurement. This is misleading the reader regarding the true effectiveness of the treatment.

The Macular Photocoagulation Study Group found that the differences in between two repeated tests were one line or more in $13 \%$ of cases and the differences were greatest in patients with visual acuity of $20 / 100$ or worse. $^{2}$ By taking up to 10 postoperative measurements, Jonas et al greatly increased the chances of a positive result. The difference between mean pre-injection 0.16 (20/ 125 or 6/36) and best mean postoperative $0.23(20 / 87$ or $6 / 26)$ was less than one line on the Snellen chart.

Their table 1 gave the mean visual acuity pre-injection and at various time intervals post-injection. At $\mathrm{l}$ and 2 months, the $\mathrm{p}$ values were 0.04 . It was unclear whether the $\mathrm{p}$ values were one or two tailed but both were described as not significant (NS) in table 1 . Multiple significance testing at each of a number of time points is generally not recommended-if it is done, some kind of adjustment to the $\mathrm{p}$ values is needed. ${ }^{34}$ Looking at the results presented in table 1 , readers might conclude that triamcinolone had a transient and doubtful beneficial effect on the visual acuity.

The authors go on to further analyse the results into improvements of three and six or more lines. The vision was tested on a Snellen chart which has irregular steps. Three or six lines do not therefore represent a constant change in visual angle (as in a logMAR chart) and therefore the analysis was confusing.

Variations in intraocular pressure of 5 or $6 \mathrm{~mm} \mathrm{Hg}$ occur diurnally in normal individuals as well as glaucomatous patients..$^{5-7}$ While there is little doubt that triamcinolone may affect the intraocular pressure, the comparison of the baseline with the highest value $(\mathrm{p}<0.001)$ was misleading as was the comparison of the highest value with that at 7 months $(\mathrm{p}<0.001)$. Of more interest might be the number of patients who had very high levels (the range extended to $64 \mathrm{~mm} \mathrm{Hg}$ ) and whether these very high intraocular pressures responded to treatment.

The authors' experience in using triamcinolone is well recognised. We congratulate them on an otherwise excellent piece of work.

D Wong, I Campbell, C Groenwald, E Lancaster

Royal Liverpool University Hospital, Prescot Street Liverpool L7 8XP, UK
Correspondence to: Mr David Wong, Royal Liverpool University Hospital, Prescot Street, Liverpool L7 8XP, UK; shdwong@liv.ac.uk

Accepted for publication 17 July 2003

\section{References}

1 Jonas JB, Kreissig I, Degenring R. Intraocular pressure after intravitreal injection of triamcinolone acetonide. $\mathrm{Br} J$ Ophthalmol 2003:87:24-7

2 Blackhurst DW, Maguire MG. Reproducibility of refraction and visual acuity measurement under a standard protocol. The Macular Photocoagulation Study Group. Retina 1989;9:163-9.

3 Altman DG. Practical statistics for medical research. London: Chapman and Hall, 1991.

4 Matthews R. The numbers don't add up. New Scientist 2003;177:28

5 Pointer JS. The diurnal variation of intraocular pressure in non-glaucomatous subjects: relevance in a clinical context. Ophthalmic Physiol Opt 1997:17:456-65.

6 De Vivero C, O Brien C, Lanigan L, et al. Diurnal intraocular pressure variation in low-tension glaucoma. Eye 1994;8(P+ 5):521-3.

7 Smith J. Diurnal intraocular pressure. Correlation to automated perimetry. Ophthalmology 1985;92:858-61.

\section{Authors' reply}

We thank Wong and colleagues very much for their interest in our study. ${ }^{1}$ We agree with them that comparing a single preoperative measurement with the best out of a series of postoperative measurements gives a tendency towards a falsely high increase in visual acuity after the triamcinolone acetonide injection. That there was an increase in visual acuity after the injection in some patients, however, may have been demonstrated in table 1 giving the visual acuity measurements before and after the application of triamcinolone acetonide. At $\mathrm{l}$ and 2 months, the difference from the preoperative values was significant with a p value of 0.04 . Unfortunately, the values are described as non-significant in what is a typographical error in the manuscript. We regret this error. The authors agree with Wong and colleagues, that the effect of triamcinolone acetonide is temporary, and that repeated injections may be necessary. In some patients, repeated intravitreal injections of triamcinolone acetonide were associated with repeated reincrease in visual acuity. ${ }^{2}$

We also agree with Wong and coworkers that comparing the preoperative intraocular pressure measurement with the highest value during the follow up again falsely increased the number of ocular hypertensive subjects after the injection. In this matter, however, it was not in favour of the treatment since it artificially increased the number of patients with unwanted side effects. Concerning the number of patients with very high intraocular pressures after the injection, a preliminary analysis shows that about $1 \%$ of the eyes injected will need filtering surgery (own data).

The authors would like to thank Wong and colleagues for constructively commenting on the study and pointing out, as did the authors, that the intravitreal injection of triamcinolone acetonide as treatment for exudative macular degeneration is still in clinical evaluation and that its therapeutic effect has not been proved so far. This may be even more important as a recent randomised study using $4 \mathrm{mg}$ triamcinolone acetonide did not demonstrate an effect on the risk of loss of visual acuity during the first year of the study in eyes with classic choroidal neovascularisation. ${ }^{3}$ A significant anti-angiogenic effect, however, was found 3 months after treatment. In view of the anti-angiogenic, anti-inflammatory, and anti-oedematous effects of intravitreal triamcinolone, further randomised trials using different doses of triamcinolone acetonide in eyes with different types of subfoveal neovascularisation may be warranted.

J B Jonas, I Kreissig, R Degenring Department of Ophthalmology, Theodor-Kutzer-Ufer, 1-3 Mannheim, Germany; jost-jonas@augen.ma. uni-heidelberg.de

Accepted for publication 17 July 2003

\section{References}

1 Jonas JB, Kreissig I, Degenring R. Intraocular pressure after intravitreal injection of triamcinolone acetonide. $\mathrm{Br} J$ Ophthalmol 2003;87:24-7

2 Jonas JB, Kreissig I, Degenring RF. Repeated intravitreal injections of triamcinolone acetonide as treatment of progressive exudative age-related macular degeneration. Graefes Arch Clin Exp Ophthalmol 2002;240:873-4

3 Gillies MC, Simpson JM, Luo W, et al. A randomised clinical trial of a single dose of intravitreal triamcinolone acetonide for neovascular age-related macular degeneration: one-year results. Arch Ophthalmol 2003; 121:667-73.

\section{Viscosurgery in diabetic vitrectomy}

Grigorian and colleagues recently recounted their experience of using viscosurgery to remove epiretinal membranes (ERMs) from eyes with proliferative diabetic retinopathy (PDR). " They concluded that "viscodissection" (injection of Healon between the fibrovascular proliferations and the retina) is safe and is equally as effective as its nonuse. On the contrary, their study shows that viscodissection is not cost effective (because of the costs of both the viscoelastic and the extra operating time), and confirms that the technique is inherently unsafe in PDR. ${ }^{2}$

The use of Healon to aid dissection of fibroglial and fibrovascular ERMs during vitrectomy was introduced in the $1980 \mathrm{~s}^{4}$ but was not widely adopted. Viscoelastic material squirting out from under the ERMs was "messy" and led to the formulation (in the 1990s) of yellow tinted Healon to aid its visualisation and simplify its removal $^{5}$ and then Healon GV (viscosity 10 times that of Healon) for adherent diabetic ERMs. ${ }^{6}$ In 1984, we began undertaking "viscodelamination" in diabetic vitrectomy. ${ }^{2} 7$ This technique was primarily directed at stripping the posterior hyaloid membrane (PHM) from detached, ischaemic and atrophic peripheral retina. Viscodelamination was especially useful in combined tractional and rhegmatogenous retinal detachments (CTRD) with very limited non-rhegmatogenous posterior vitreous detachment (PVD) present. Because of the prohibitive cost of Healon, methylcellulose $1 \%$ was injected in the majority of eyes.

To summarise our experience, stripping of the PHM usually proceeded uneventfully during slow pressurisation of the closed retrohyaloid compartment by viscoelastic, as did separation of any loosely adherent, sparsely vascularised ERMs that were contained within the peripheral vitreous cortex. ${ }^{2}$ In well photocoagulated eyes, the separation 
sometimes continued posteriorly, culminating in a complete PVD. In the case of more adherent fibrovascular ERMs, their "viscoelevation" sometimes occurred through stretching of the vascular and glial tent pegs connecting the ERMs to the retina. The PHM and ERMs could then be removed en bloc using the suction cutter. However, instead of stretching, the vascular connections between the ERM and the retina tended to be disrupted. Avulsion generally occurred at the point of greatest weakness at the origins of neovascular outgrowths from the retinal veins. Although correlating with ERM vascularity and with the density of neovascular outgrowths from the retina, ERM retinal adherence was unpredictable, and bleeding was ultimately an inevitable consequence of the perpendicular hydraulic forces necessary to effect peeling of more adherent ERMs. Fortunately, the bleeding from side punctures in the retinal veins was constrained by the viscoelastic (so called "haemorrhagic confinement" $\left.{ }^{2}\right)^{8}$ and a high ambient intraocular pressure during the surgery. However, as was predictable in theory, but again unpredictable in practice, the hydraulic tension sometimes disrupted the retina ahead of, and instead of, peeling the ERMs. Furthermore, recurrent fibroglial membranes were sometimes observed later even in eyes where viscodelamination had proceeded uneventfully. This has been attributed to the difficulty in completely removing viscoelastic from the retinal surface, with preretinal retention of growth factors. ${ }^{2}{ }^{3}$ Not for nothing is one viscoelastic mixture marketed as Viscoat. We had discontinued viscosurgery in PDR by 1988 in favour of purely mechanical methods that minimise ERM elevation. ${ }^{3}$

Fifteen years on and Grigorian and colleagues have clearly come to a very different conclusion from ours despite reporting a considerable excess of iatrogenic posterior retinal breaks during, and recurrent detachment after, viscosurgery. ${ }^{1}$ By back calculation from their assiduously collected data, it appears that 20 posterior breaks were induced in 65 eyes undergoing viscodissection compared with four in 89 eyes having conventional surgery. This trend was confirmed in groups of eyes with pathology of similar ("relatively high") complexity. Thus, there were 10 iatrogenic posterior breaks in 34 viscodissections in eyes in the range C4-6 compared with three in 26 conventional operations. (It is acknowledged that Grigorian et al state that the complexity score "does not account for the degree of adhesion," neither was it "a good predictor of the amount of traction necessary to dissect a membrane.") The intraoperative problems appear to have been reflected in the ultimate outcomes. After 6 months of follow up, for example, a detached retina was evident in seven of 43 eyes $(16 \%)$ in the viscosurgery group compared with three of 58 eyes (5\%) undergoing conventional surgery. Furthermore, although eyes with CTRD seemed to fare well whether or not Healon was used, this was not the case in eyes with tractional detachments (with or without vitreous haemorrhage). Six of 30 eyes (20\%) had a detached retina 6 months after viscodissection compared with only two of 37 eyes (5\%) after conventional surgery. Indeed, most of the data favoured conventional surgery. Lower viscosity Healon was proposed as a future means of reducing the frequency of iatrogenic breaks, but this is unlikely to be helpful in their attempt to achieve the impossible-that is, a worthwhile increase in the ease and speed of ERM removal without an unacceptable added risk of retinal haemorrhage, tears, and scarring. Better by far would be to avoid viscoelastics altogether.

D Mcleod

University of Manchester, Manchester Royal Eye Hospital, Oxford Road, Manchester M13 9WH, UK david.mcleod@man.ac.uk

Accepted for publication 21 July 2003

\section{References}

1 Grigorian RA, Castellarin A, Fegan R, et al. Epiretinal membrane removal in diabetic eyes: comparison of viscodissection with conventional methods of membrane peeling. $\mathrm{Br} J$ Ophthalmol 2003;87:737-41.

2 McLeod D, James CR. Viscodelamination at the vitreoretinal juncture in severe diabetic eye disease. Br J Ophthalmol 1988;72:413-19.

3 Charles S. Techniques and tools for dissection of epiretinal membranes. Graefes Arch Clin Exp Ophthalmol 2003;241:347-52.

4 Stenkula S, Ivert I, Gislason I, et al. The use of sodium-hyaluronate (Healon) in the treatment of retinal detachment. Ophthalmic Surg $1981 ; 12: 435-43$.

5 Stenkula S, Ivert I, Berglin L, et al. Healon yellow as a surgical tool in maneuvering intraocula tissues. Ophthalmic Surg 1992;23:708-710.

6 Crafoord S, Stenkula S. Healon GV in posterior segment surgery. Acta Ophthalmol (Copenh) 1993;71:560-1.

7 Barry PJ, Hiscott PS, Grierson I, et al. Reparative epiretinal fibrosis after diabetic vitrectomy. Trans Ophthalmol Soc UK 1985; 104:285-96.

8 Folk JC, Packer AJ, Weingeist TA, et al. Sodium hyaluronate (Healon) in closed vitrectomy. Ophthalmic Surg 1986;17:299-306.

\section{CORRECTION}

In the article by Brodsky et al in the February issue (Br J Opthalmol 2004;88:268-272), a portion of the text within fig 2 was incorrectly labelled. The label under "+ Superior rectus contracture" is currently printed as "Compensatory head tilt away from side of fixing eye." It should have been printed as "Compensatory head tilt toward the side of the fixing eye."

\section{NOTICES}

\section{Cataract surgery}

The latest issue of Community Eye Health (No 48) discusses a solution to reduce worldwide cataract blindness, including sutureless non-phaco cataract surgery. For further information please contact: Journal of Community Eye Health, International Resource Centre, International Centre for Eye Health, Department of Infectious and Tropical Diseases, London School of Hygiene and Tropical Medicine, Keppel Street, London WCIE 7HT, UK (tel: +44 (0)207612 7964; email: Anita.Shah@1shtm. ac.uk; website: www.jceh.co.uk). Annual subscription (4 issues) UK£28/US\$45. Free to developing country applicants.

\section{Elimination of avoidable blindness}

The 56th World Health Assembly (WHA) considered the report on the elimination of avoidable blindness (doc A56/26) and urged Member States to: (1) Commit themselves to supporting the Global Initiative for the Elimination of Avoidable Blindness by setting up a national Vision 2020 plan by 2005; (2) Establish a national coordinating committee for Vision 2020, or a national blindness prevention committee to help implement the plan; (3) Implement the plan by 2007; (4) Include effective monitoring and evaluation of the plan with the aim of showing a reduction in the magnitude of avoidable blindness by 2010; (5) To support the mobilisation of resources for eliminating avoidable blindness. The WHA also urged the Director-General to maintain and strengthen WHO's collaboration with Member States and the partners of the Global Initiative for the Elimination of Avoidable Blindness as well as aid in the coordination and support of national capability.

\section{XVth Meeting of the International Neuro-Ophthalmology Society}

The XVth Meeting of the International Neuro-Ophthalmology Society will take place 18-22 July 2004, in Geneva, Switzerland. Further details: Prof. A Safran, University Hospital Geneva, c/o SYMPORG SA, Geneva (fax: +4122 839 8484; email: info@symporg.ch; website: www.symporg.ch).

\section{4th International Congress on Autoimmunity}

The 4th International Congress on Autoimmunity will take place 3-7 November 2004 in Budapest, Hungary. The deadline for the receipt of abstracts is 20 June 2004. Further details: Kenes International Global Congress Organisers and Association Management Services, 17 Rue du Cendrier, PO Box 1726, CH-1211 Geneva l, Switzerland (tel: +41 22908 0488; fax: +4122732 2850; email: autoim04@kenes. com; website: www.kenes.com/autoim2004).

\section{International Congress for Eye Research}

The XVI International Congress for Eye Research will be held on 29 August-3 September 2004 in Sydney, Australia. For further information, please contact: icer2004@ tourhosts.com.au (website: www.tourhosts. com.au/icer2004). 